INTERNATIONAL MONETARY FUND

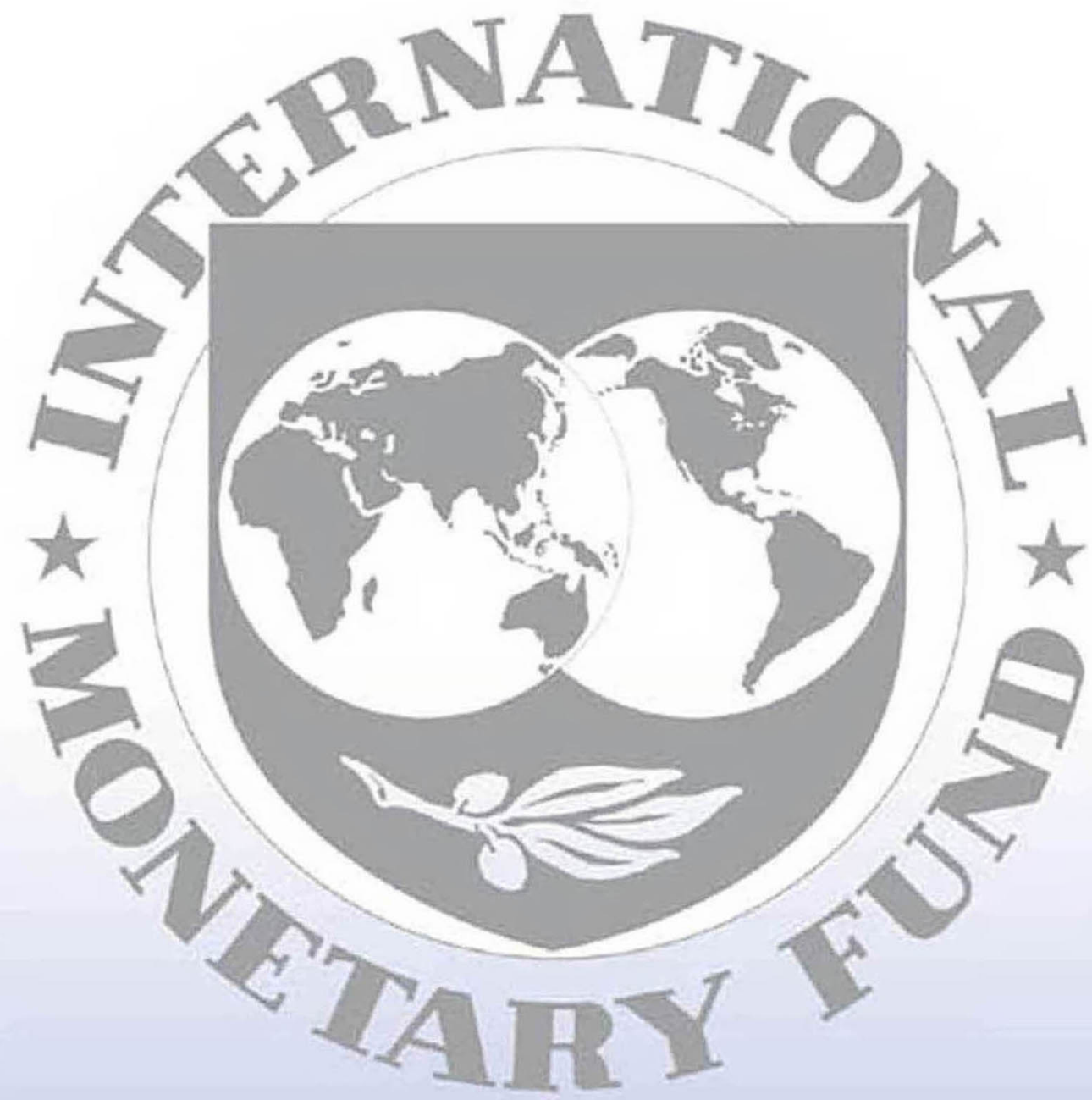

Staff
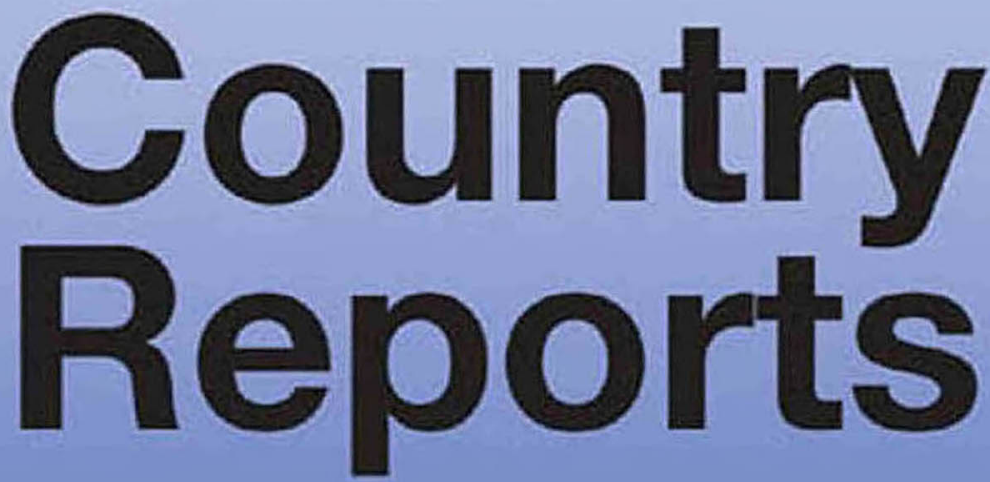


\section{Democratic Republic of the Congo: 2012 Article IV Consultation-Staff Report; Public Information Notice on the Executive Board Discussion; and Statement by the Executive Director for the Democratic Republic of the Congo}

Under Article IV of the IMF's Articles of Agreement, the IMF holds bilateral discussions with members, usually every year. In the context of the 2012 Article IV consultation with the Democratic Republic of the Congo, the following documents have been released and are included in this package:

- The staff report for the 2012 Article IV consultation, prepared by a staff team of the IMF, following discussions that ended on June 21, 2012, with the officials of the Democratic Republic of the Congo on economic developments and policies. Based on information available at the time of these discussions, the staff report was completed on September 4, 2012. The views expressed in the staff report are those of the staff team and do not necessarily reflect the views of the Executive Board of the IMF.

- $\quad$ A Public Information Notice (PIN) summarizing the views of the Executive Board as expressed during its September 24, 2012, discussion of the staff report that concluded the 2012 Article IV consultation.

- $\quad$ A statement by the Executive Director for the Democratic Republic of the Congo.

The documents listed below have been or will be separately released.

$$
\begin{aligned}
& \text { Joint Staff Advisory Note of the Poverty Reduction Strategy Paper } \\
& \text { Poverty Reduction Strategy Paper }
\end{aligned}
$$

The policy of publication of staff reports and other documents allows for the deletion of market-sensitive information.

Copies of this report are available to the public from

International Monetary Fund • Publication Services $70019^{\text {th }}$ Street, N.W. • Washington, D.C. 20431

Telephone: (202) 623-7430 • Telefax: (202) 623-7201

E-mail: publications@imf.org Internet: http://www.imf.org

\section{International Monetary Fund Washington, D.C.}




\section{INTERNATIONAL MONETARY FUND}

\section{DEMOCRATIC REPUBLIC OF THE CONGO}

\section{STAFF REPORT FOR THE 2012 ARTICLE IV CONSULTATION}

\section{KEY ISSUES}

Context: Despite the difficult global economic environment, limited external financial support, and uncertainty surrounding national elections in November 2011, macroeconomic performance in the Democratic Republic of the Congo (DRC) was strong in 2010-11. An improvement in economic policies underpinned this performance but recent shortcomings in the formulation of the 2012 budget and potential slippages related to security spending could reverse hard-won progress in reducing fiscal dominance and consolidating macroeconomic stability.

Buffers need to be strengthened: The DRC's experience during the global financial crisis in 2009 and the downside risks from the current weakness in the world economy, argue for a further buildup of macroeconomic buffers. Although the fiscal position has improved through consolidation and debt relief, it remains vulnerable to spending pressures from the deteriorating security situation in the eastern provinces, which is exacerbated by limited financing options and slow progress in mobilizing more domestic revenue. Gross international reserves have also risen, however, they remain below the level required to cushion the economy from the impact of external shocks.

Stronger institutional and administrative capacity is essential: The authorities' recent efforts to improve macroeconomic stability under difficult circumstances must be commended. To maintain this stability over the medium term will require a stronger institutional setting with a well-defined fiscal framework targeting appropriate fiscal anchors, supported by strong public financial management; an independent central bank capable of effectively carrying out monetary policy; a deeper and adequately supervised financial sector; and a business environment conducive to attracting investment and diversifying export capacity to reduce external vulnerability. 
Approved By Michael Atingi-Ego and Christian Mumssen
Discussions took place in Kinshasa June 7-21, 2012. The staff team comprised Messrs. York (head), Fischer and Jahjah and Ms. Lundgren (all AFR), and Mr. Arnold (FAD). Mr. N'Sonde (OED) and staff from the World Bank and African Development Bank also participated in the discussions. The mission met with the President of the Senate Léon Kengo Wa Dondo, the President of the National Assembly Aubin Minuku, Prime Minister Matata Ponyo Mapon, Vice-Prime Minister and Minister of Budget Daniel Mukoko Samba, Minister Delegate to the Prime Minister for Finance Patrice Kitebi Kibol Mvul, Central Bank of Congo Governor Jean-Claude Masangu, other senior ministers and government officials, and representatives from the private sector, civil society, and the donor community.

\section{CONTENTS}

BACKGROUND

CONTEXT, OUTLOOK, AND RISKS

A. Outlook and Risks

POLICY DISCUSSIONS

A. Anchoring Fiscal Policy $\underline{13}$

B. Enhancing the Efficacy of Monetary Policy $\underline{14}$

C. Making the Economy More Robust $\underline{17}$

STAFF APPRAISAL $\underline{19}$

\section{TABLES}

1. Selected Economic and Financial Indicators, 2009-15__ 22

2. Balance of Payments, 2010-15__ $\underline{23}$

3a. Central Government Financial Operations, 2010-15__ 24

3b. Central Government Financial Operations, 2010-15__ 25

4. Monetary Survey, 2009-13 __ 26

5. Risk Assessment Matrix__ $\underline{27}$

6. Fiscal Position, 2011-12 _ $\underline{28}$

7. Financial Soundness Indicators, 2003-June $2012 \_\underline{29}$ 


\section{FIGURES}

1. Recent Economic Developments $\underline{6}$

2. Macroeconomic Buffers, 2009 and 2012 Z

3. Policy Performance 8

4. Tail-Risk Scenarios-Plunging Commodity Prices and Domestic Conflict 11

5. Foreign Currency Deposits, 2005 and 2010 $\underline{16}$

6. Broad Money, 2005 and 2010 $\underline{16}$

\section{ANNEXES}

I. Updated Debt Sustainability Analysis $\underline{30}$

II. Performance Under the Extended Credit Facility Arrangement 44

III. International Competitiveness $\underline{45}$

IV. Technical Assistance to the DRC 47

V. Reserve Adequacy Estimates from the IMF Template $\underline{48}$

VI. Fiscal Implications of Current Mining-Sector Tax Policies $\underline{49}$

VII. Improving Domestic Fuel Pricing $\underline{50}$

VIII. The Efficacy of Monetary Policy $\underline{57}$ 


\section{BACKGROUND}

1. In the past few years, economic performance in the Democratic Republic of the Congo (DRC) has improved markedly, although progress in alleviating poverty and meeting the Millennium Development Goals has lagged. Prudent macroeconomic policies and structural reforms have underpinned this performance and led to the DRC receiving debt relief under the enhanced Heavily Indebted Poor Countries (HIPC) Initiative in mid-2010. This debt relief-the largest amount to any eligible HIPC countryreduced the DRC's external debt burden from about 136 percent of GDP in 2009 to about 35 percent at the end of 2010. Regrettably, the level of poverty probably remains high-about 70 percent of the population - and the DRC is unlikely to meet any of the MDGs. ${ }^{1}$

\section{In the November 2011 elections, President Kabila was reelected and his coalition partners won a majority in} parliament. This brought with it a measure of political and social stability and expectations that the thrust of economic policies would continue. While some key reforms have been pushed forward, poor performance in the management of public finances, external debt, and natural resources has emerged. Along with the deteriorating security situation in the eastern provinces, these weaknesses could undermine recent progress and jeopardize the

\footnotetext{
${ }^{1}$ The most recent official money-metric poverty data is from 2005; updated data is expected in early 2013. The current IMF-World Bank joint staff assessment of poverty and social indicators are contained in a Joint Staff Advisory Note on the authorities' Poverty Reduction and Growth Strategy (PRS) paper (see also Figure III.2).
}

completion of the fourth and fifth reviews of the authorities' Extended Credit Facility (ECF) arrangement. $^{2}$

\section{In the context of the Article IV} consultations, the authorities have been receptive to the IMF's advice, although they have addressed the recommendations from the previous discussions with varying amounts of determination and vigor. The cornerstone of their efforts has been fiscal consolidation, where they have responded positively to the IMF's call for spending restraint and the end to inflationary fiscal financing from the Central Bank of the Congo (BCC). Monetary and financial sector policies have moved in the right direction but progress has been slower than expected. Likewise, structural reforms to enhance public financial management (PFM) and natural resource management are being undertaken, although the political will to tackle the most difficult issues has waned. The authorities are making concerted efforts to implement the 2010 safeguards assessment recommendations, including the BCC's move to International Financial Reporting Standards.

\section{The 2012 Article IV discussions kept} the focus on many of these same issues, but shifted the timeframe from the short-tomedium term. The staff team exchanged views with the authorities and stakeholders on

\footnotetext{
2 The IMF's Executive Board approved the DRC's 3-year ECF arrangement in December 2009 and the third review was completed in April 2011. The fourth review (planned for late 2011) was delayed because of lack of progress in structural reform.
} 
the following main issues: anchoring fiscal policy to strengthen credibility and sustainability; improving the efficacy of monetary policy in the context of serious constraints; and making the economy more robust and less vulnerable to shocks.

\section{CONTEXT, OUTLOOK, AND RISKS}

The economy shows remarkable resilience

5. Macroeconomic performance in the DRC was strong during 2010-11 even though the global economy remained weak, external financial support was limited, and the national elections in November 2011 created significant uncertainty. Solid progress was made toward meeting many of the country's medium-term economic objectives.

- The DRC's weak financial linkages with the euro area countries have largely shielded it from the turmoil there. Strong trade and investment inflows from non-European countries, driven mainly by the mining sector, have helped support economic activity in the past few years. This activity broadened to the manufacturing and tertiary sectors (telecommunications, beverages, construction) and real GDP growth expanded by about 7 percent in 2010-11 (Table 1, Figure 1).

- Inflation was above the BCC's single-digit target in 2011, mainly because of high global food and fuel prices early in the year but has since been on a downward trend. Domestic prices bumped up at the beginning of 2012 alongside the introduction of the value-added-tax, but inflation has regained its downward path to just below 10 percent through July (Figure 1).
- The external sector improved significantly as a result of HIPC debt relief but remains vulnerable to the volatile terms of trade, high import dependency on food and capital goods, narrow export base, and high level of dollarization of the banking sector. The DRC also continues to be at high-risk of debt distress because of the inclusion of the public guarantee on external borrowing under a SinoCongolese joint venture (Sicomines, Annex I). The current-account deficit widened in 2011 (about 111/2 percent of GDP) as commodity prices weakened from their high levels in 2010 and the value of imports increased, especially for food, fuel, and capital goods (Table 2).

The rebuilding of macroeconomic buffers supported recent developments

6. These buffers were improved along four of five dimensions (Figure 2).

- Fiscal dominance has been reduced in the past few years, owing to the government's expenditure restraint and commitment to zero (net) financing of the budget from the $B C C$ (excluding the use of deposits from HIPC debt relief and several grants saved from 2010). This has helped break a vicious inflation-exchange rate depreciation cycle and brought the domestic fiscal deficit down from 21/2 percent of GDP in 2009 to a projected deficit of 0.9 percent of GDP in 2012 (Tables 3a and 3b, Figure 3). 


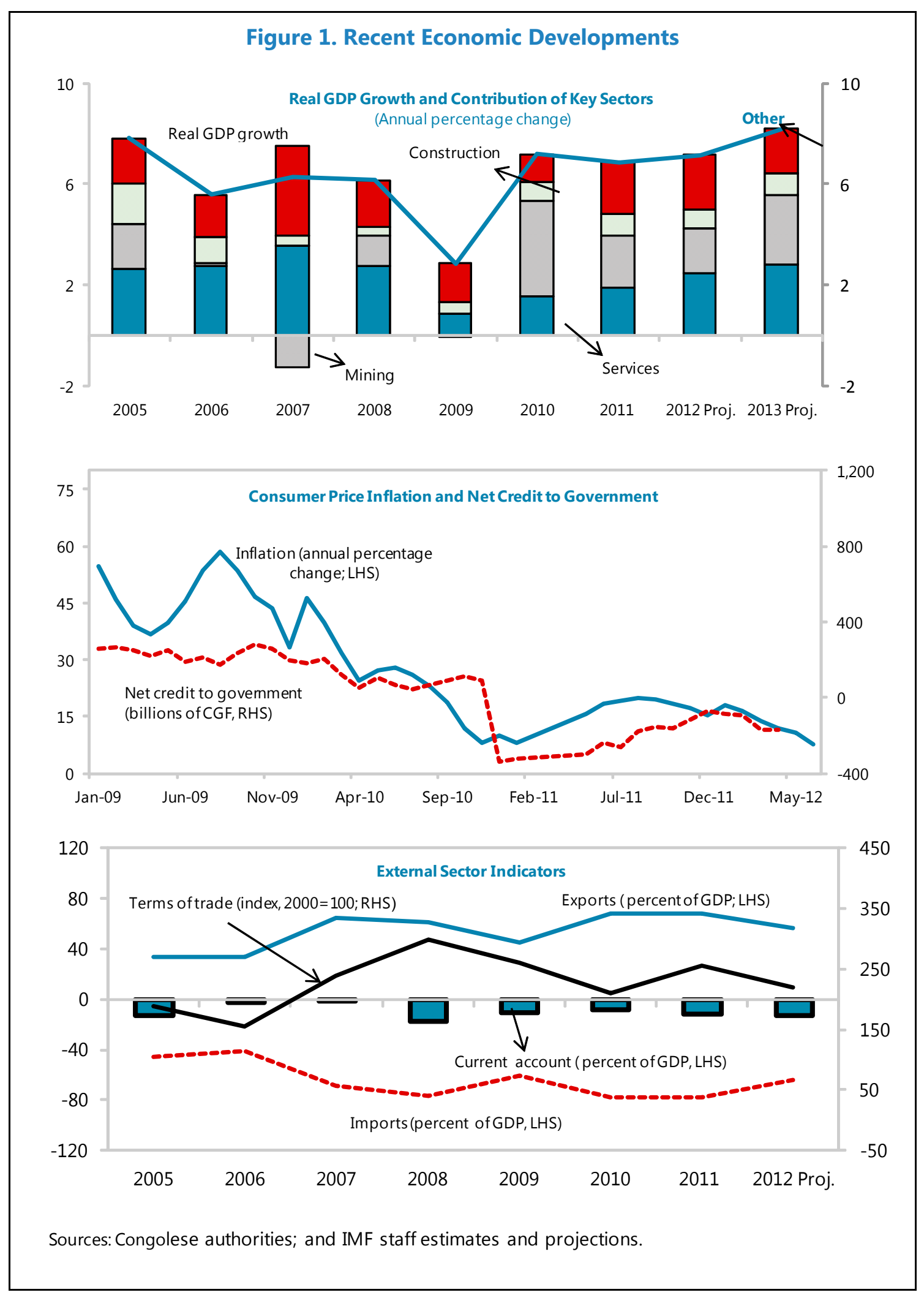

CInternational Monetary Fund. Not for Redistribution 
Figure 2. Macroeconomic Buffers, 2009 and 2012

(Units indicated)

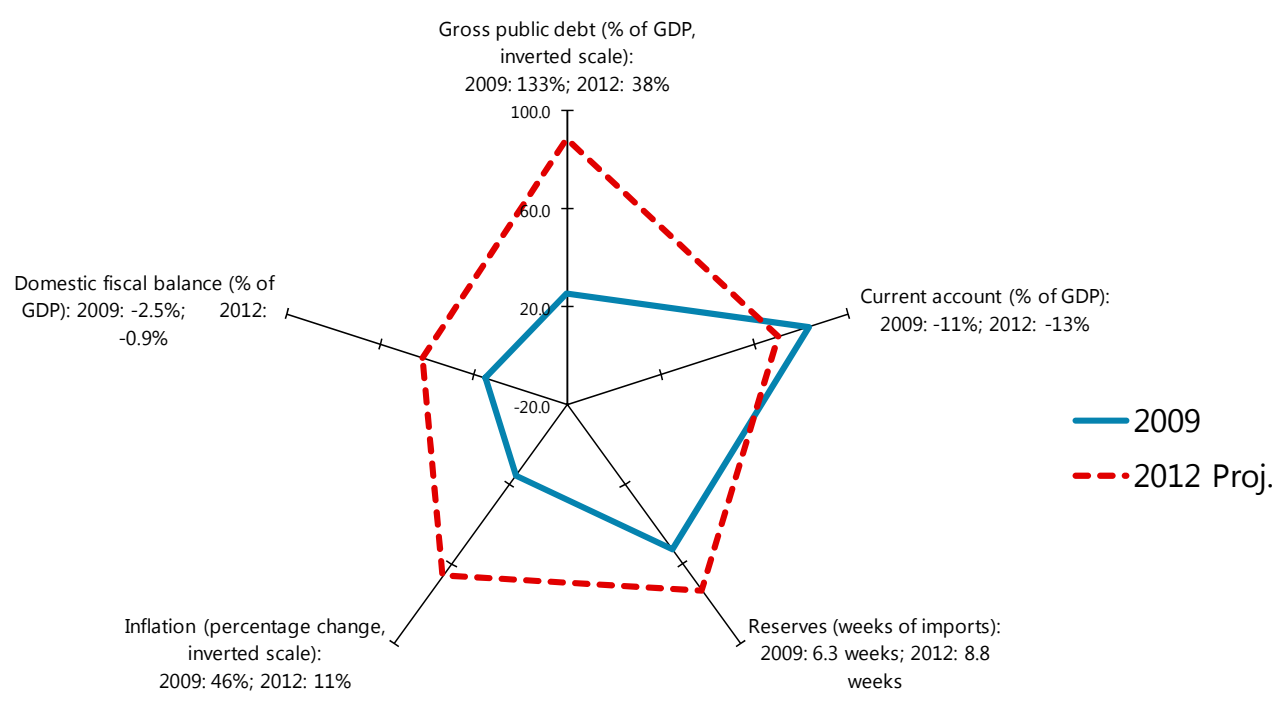

Sources: Congolese authorities; and IMF staff estimates and projections.

- Expenditure control has been necessary to address the limited external financial support to the DRC - reflecting donors concerns over governance, especially in the natural resource sector-and slow progress in raising more domestic revenue. Recently, however, weaknesses in the budget process, inflated revenue projections, and a corresponding large expenditure envelope point to the need to bolster and better entrench PFM reforms (Annex II).

- The improvement in the fiscal situation has also helped the BCC control liquidity and fight inflation. The BCC contains inflation through the issuance of central bank bills but data and capacity problems, as well as political pressures have made it hesitant at times to proactively tighten monetary policy. It has aggressively reduced its policy interest rate faster than justified by inflation developments, leading to relatively low real interest rates (Figure 3 ). More recently, the BCC's own budgetary constraints prompted it to reduce its stock of central bank bills in order to reduce interest costs, leading to an unwarranted increase in liquidity. 


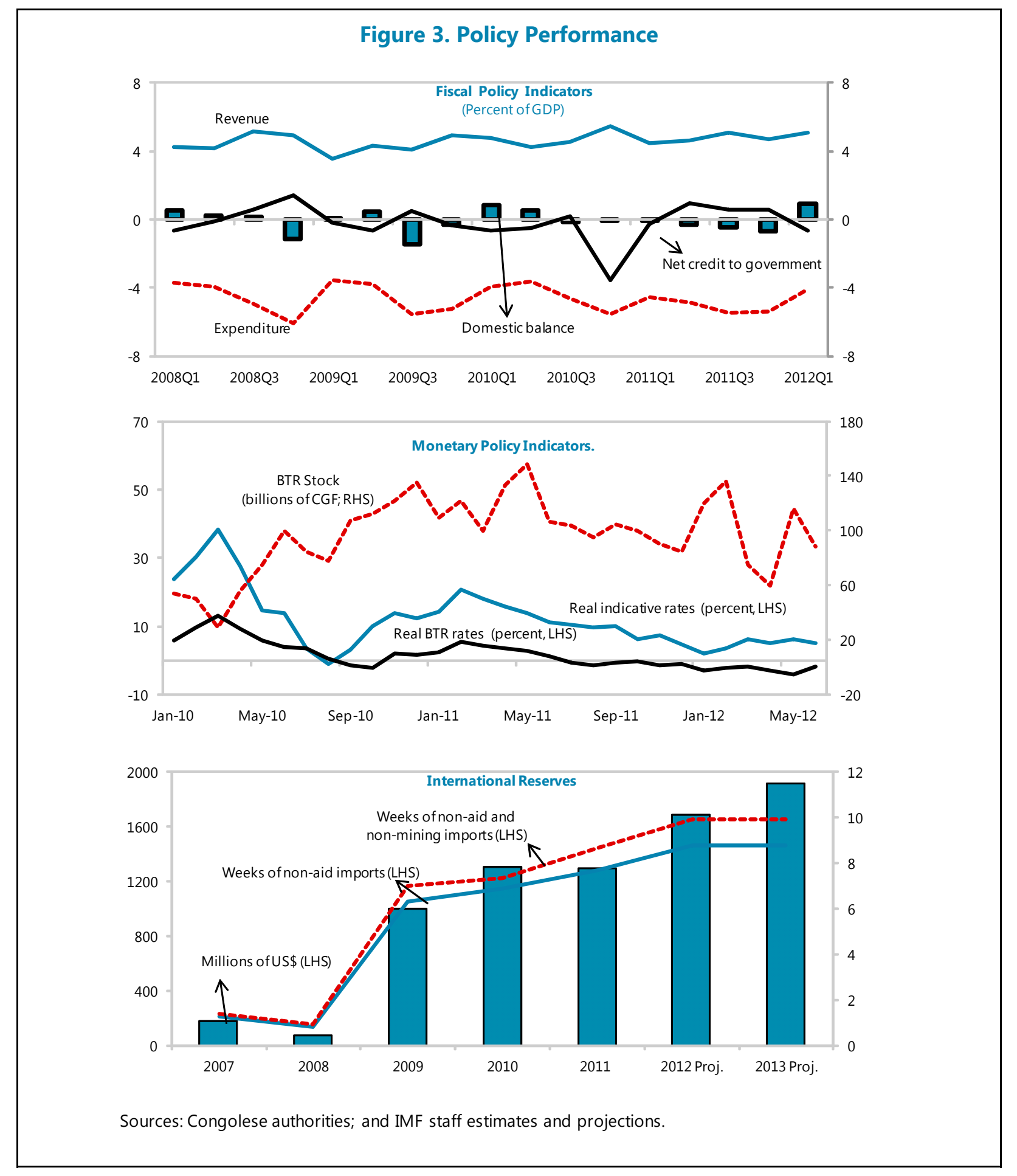

- External stability has been strengthened through HIPC debt relief and a buildup of international reserves (from about 6.3 weeks of import cover in 2009 to a projected 8.8 weeks this year, Figure 3), which provides a larger cushion against external shocks. Notwithstanding this progress, an even larger buffer is needed to bolster the DRC's external stability, including from a projected widening of the 
current account deficit and growing dollardeposits in the banking system. External borrowing should be consistent with maintaining external debt sustainability and only on highly concessional terms. The current account deficit has increased in the past few years, reflecting the volatility of the terms of trade (rather than deterioration in the DRC's international competitiveness), high imports related to mining sector and infrastructure investments, and better accounting of income flows. Export volumes (mainly commodities) also expanded significantly in the same period. But the recent deterioration of the current account might also be linked to the ongoing difficult business environment, which influences the DRC's international competiveness more than the level of the real effective exchange rate (REER). Indeed, according to the staff and authorities' assessment, the REER is probably in line with the fundamentals (Annex III).

\section{Although progress in structural} reform has been mixed, some reforms in key areas have been supportive of better economic performance. In particular, the authorities have been moving methodically, if unevenly, to put in place their Strategic Plan for Public Finance Reform with technical assistance (TA) from the IMF, World Bank, and

\section{A. Outlook and Risks}

The short-term outlook appears relatively benign

\section{Recent global and domestic} developments have not dampened investors' appetite for ramping up nearterm production, especially in the mining sector. On present projections, mining-related development partners (Annex IV). Much of the IMF TA focuses on the fiscal planning process, helping to build tools for better macrofiscal forecasting and moving toward a mediumterm budget framework with expenditure priorities guided by a new Poverty Reduction Strategy (PRS).

8. The authorities implemented a large number of measures supported by the World Bank to strengthen governance in the natural resource sector and they recently submitted the instruments to adhere to the Organization for the Harmonization of African Business Law (OHADA) in Dakar. The BCC intervened in mid-2010 to address problems with a large commercial bank to avoid a systemic crisis, while extending its efforts toward bolstering financial supervision and hastening the development of the banking sector. The BCC has also completed a pension reform and is in the process of shedding non-core activities to better focus on its mandate.

\section{Still, other reforms have lagged and} new issues have emerged, including weaknesses in PFM (i.e., an unrealistic 2012 budget), external debt management (emergence of external payments arrears) and problems in ensuring accountability and transparency in the operations of state-owned enterprises (SOEs) in extractive industries.

investments will continue to grow in 2012-16, providing a major impetus to real GDP growth, which could exceed 7 percent this year and 8 percent in 2013. While some of these investment plans could be scaled back if conditions worsen, any softening could be 
partially offset by the Sicomines project whose implementation has only recently begun.

\section{The strong investment cycle will} invariably lead to a widening of the current account deficit as a consequence of heavy capital imports. The larger current account deficit would also reflect the DRC's rules on accelerated depreciation of investments for tax purposes, which allow for large tax deductions of investments that, consequently, increase income outflows in the current account. Fortunately, the deficit should be adequately financed over the medium term, by the strong inflows of direct investment from these mining projects (including Sicomes).

\section{The prospects for an expansion of} mineral exports over the medium term should lead to higher foreign-exchange earnings and with them, opportunities for the BCC to accumulate further international reserves. The authorities' objective of 10 weeks of import coverage therefore is achievable, although the staff's estimates using the IMF template for reserve adequacy suggest this objective should be set much higher to ensure external stability (Annex $\mathrm{V}$ ).

\section{Rising international food prices,} however, may also complicate the task of the BCC. Although the magnitude will depend on the price increases on crops most relevant to domestic consumption, recent history points to significant pass-through from international food prices to inflation. If realized, this may jeopardize the achievement of the BCC's single-digit inflation target this year and slow down the increase in international reserves. ${ }^{3}$

\section{But downside risks are significant}

14. The outlook is subject to a number of downside risks, a couple of which could have a severe impact on the DRC. Some of the risks stem from the potential for further weakness in the euro area that spills over into lower global growth, with adverse implications for already weakening commodity prices. Other risks could be homegrown; in particular, those stemming from fiscal slippages given expenditure rigidities if overly optimistic tax revenue projections are not realized, spending pressures from an escalation of the recent conflict in the eastern part of the country, or a reversal of governance reforms that put a freeze on investment (Table 5).

\section{Although the DRC authorities have made concerted efforts to rebuild the macroeconomic buffers, there is a strong rationale making them even bigger. A steep drop in commodity prices comparable in scope to the decline in 2009 and an escalating conflict that requires a substantial increase in security-related spending would have serious implications. Together with the authorities, the staff elaborated on these shock (or tail-risk) scenarios.}

- Plunging commodity prices: A 40 percent decline in commodity prices relative to the baseline (equal to two standard deviations

\footnotetext{
${ }^{3}$ The government does not provide food subsidies so beyond the effect on inflation there would not be a direct fiscal impact of rising food prices. If, however, the government responded by cutting import tariffs or the VAT rate on key food imports, it could reduce revenue somewhat.
} 
from the historical average) in 2013, with a partial reversal toward the baseline in 2014 would lead to a sharp slowdown in mining investment and production. In the first year of the shock, this could knock 6 percentage points off real GDP growth and cause the current account deficit to balloon by more than 12 percent of GDP, and lower fiscal revenue by nearly 4 percent of GDP (Figure 4). The exchange rate would likely depreciate substantially, which in turn could lead to a spike in inflation.

Figure 4. Tail-Risk Scenarios-Plunging Commodity Prices and Domestic Conflict

(Deviation from the Baseline)
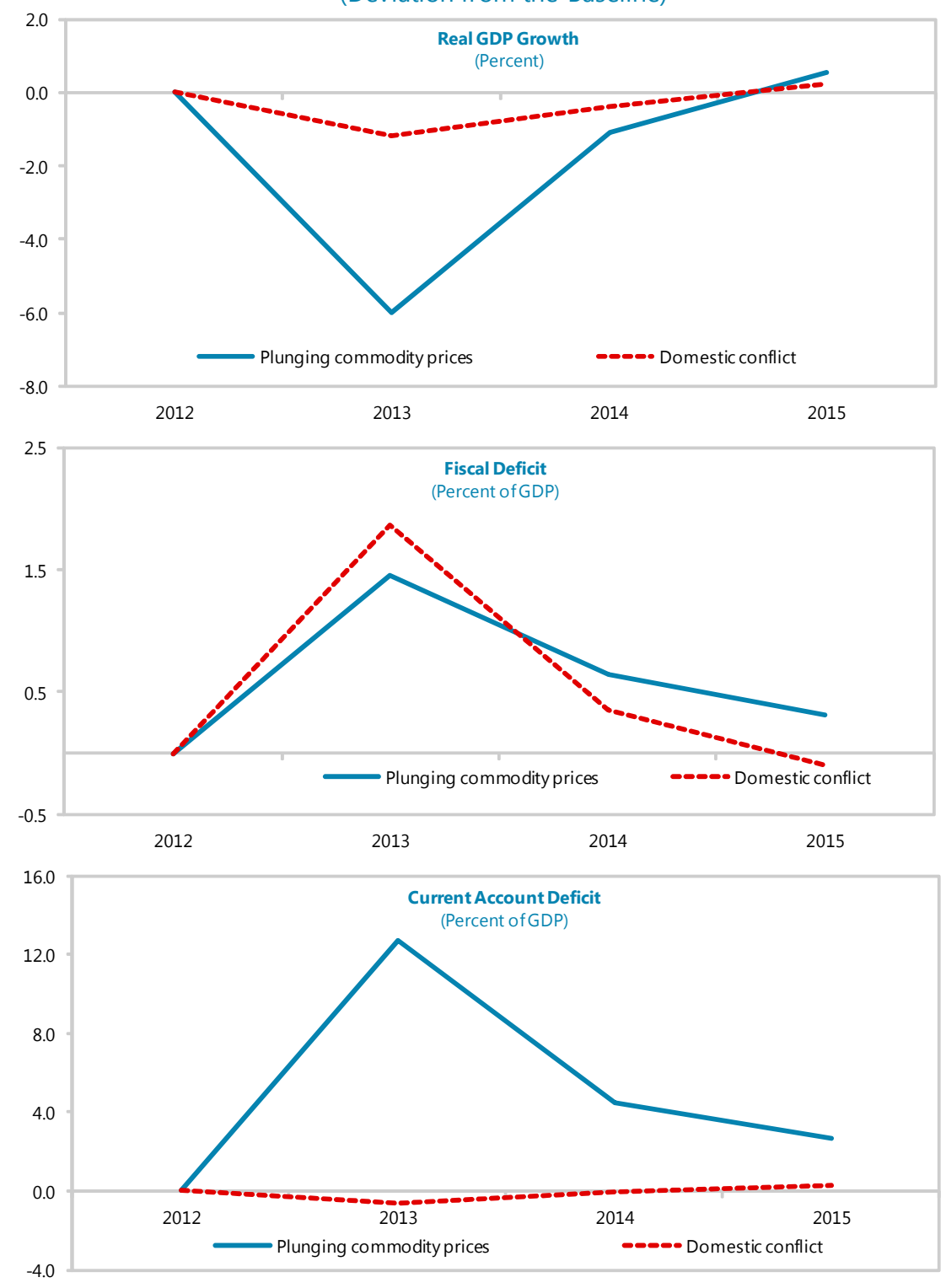

Sources: Congolese authorities; and IMF staff estimates and projections. 
- An escalation of conflict in eastern DRC, similar to civil war in the mid-2000s. The direct impact on growth from increased insecurity could be muted because the country's major mines lie outside the affected areas, though it is difficult to gauge the impact on investment. Also, an increase in security-related spending equal to 3 percent of GDP financed by the central bank in the absence of other financing options likely would have a smaller impact on growth and inflation than a sharp fall in commodity prices, assuming that the government cuts other spending items. At the same time, large cuts would likely have an adverse effect on budget execution and the quality of spending. In particular, sizable cuts to infrastructure spending would have a negative impact on growth over the medium term. ${ }^{4}$

\section{Authorities' views}

\section{The authorities appreciated the} staff's candid assessment of the economic outlook and agreed with the risk assessment. Indeed, they noted that rebuilding the macroeconomic buffers must be a primary objective of their economic program.
They emphasized that the lessons learned from the global financial crisis in 2009- when commodity prices plunged, the exchange rate depreciated rapidly, inflation accelerated, and international reserves were nearly depletedwere deeply ingrained. Having said that, they did feel the economy is in a stronger position today to absorb external shocks because of better economic policy implementation and reduced security risks, notwithstanding the current problems in the eastern region.

\section{Overall, the authorities also agreed} with the staff's analysis of the exchange rate and international competitiveness, and the need to accumulate more foreign reserves. However, they argued that reserve accumulation needed to be balanced against the possible implications from foreign exchange purchases on the exchange rate, whose changes immediately affect inflation. In light of the high cost of elections, debt service due after HIPC debt relief, and the reduction in balance of payments support, the authorities' saw that building more reserves could have led to adverse macroeconomic effects. On external debt, the authorities felt that the risks related more to the use of external financing in terms of inefficient and wasteful public investment than to debt distress.

with low and stable inflation, was the main focus of the discussions. The staff relied heavily on the experience across sub-Saharan Africa and other low-income countries, in providing its advice.

items, compared with near 100 percent for some others (including political institutions).

\footnotetext{
${ }^{4}$ Fiscal restraint has already resulted in ad hoc spending cuts that have adversely affected budget execution and the quality of spending, with persistently poor execution rates for many important
} (continued) 


\section{A. Anchoring Fiscal Policy}

19. After a few years of relatively good performance, debt relief, and a change in government, discussions focused on the direction of fiscal policy. The staff expressed concern about a shift in the fiscal policy approach, as evidenced by the 2012 budget, leading to considerations about how to consolidate recent progress and sustain it over the medium term. Establishing a credible fiscal anchor would help.

20. Over the short term, fiscal policy must be aimed at maintaining expenditure control-the foundation for the authorities' program so far-while re-focusing attention on the composition of the budget and its priorities. Raising more domestic resources by broadening the tax base is essential in light of the time-profile of tax revenue from the natural resource sector as a result of mineral tax policies (Annex VI), and limited external financial support. Targeting a domestic fiscal deficit of about 1 percent of GDP this year is appropriate and consistent with medium-term fiscal sustainability (Table 6). ${ }^{5}$

21. In this context, the overall resource envelope of the 2012 budget submitted by

\footnotetext{
${ }^{5}$ Although the "fiscal space" measure calculated for the DRC is positive (about 1 percent of GDP over the medium term), it probably does not accurately reflect the government's capacity to respond to shocks with looser fiscal policy because the DRC lacks access to international capital markets or other forms of financing. The fiscal space measure is the difference between the current primary balance and the hypothetical balance that would have to be realized every year for the next 20 years to achieve a particular debt-to-GDP ratio target (30 percent of GDP in the case of the DRC).
}

\section{the government and approved by the} parliament is overly ambitious. The budget contains domestic revenue projections about 3 percent of GDP higher than what can be considered feasible in the absence of credible tax policy measures or administrative reforms to support them. ${ }^{6}$ Higher spending associated with this revenue target-including for nondiscretionary items such as wages, and current spending on political institutions that tend to be fully executed-could affect the quality of spending and, along with increased securityrelated outlays, raise the potential for significant expenditure slippages. Although the government's desire to expand the budget envelope is understandable, depriving the budget of its disciplining role is not an adequate response to the country's substantial development needs. Rather, it risks reversing hard-won progress in consolidating the fiscal position.

\section{Instead, the staff argued that the} authorities should publicly declare their commitment to zero central-bank-financing of the budget, while also targeting the domestic fiscal balance as the preferred measure of the fiscal stance. This would help anchor expectations; support the BCC, whose independence is still limited; and break the link between fiscal policy and inflation. Also, the staff indicated that it is premature for the DRC to use alternative measures of the fiscal stance, such as a non-resource primary balance, or

\footnotetext{
${ }^{6}$ The budget also includes higher projections for project grants but this does not pose a fiscal risk since most of this spending is executed directly by donors. If these grants do not materialize the spending is not executed.
} 
adopt a fiscal rule in its place. International experience suggests that properly employing more sophisticated measures of the fiscal position or rules, in conjunction with a medium-term fiscal framework or fiscal rule, requires better quality statistics, especially for the natural resource sectors and strong PFM. Although the DRC is moving in the right direction in this respect, progress across the relevant parts of the government is uneven and often slow. A number of weaknesses still exist in public accounting, expenditure-chain management, the budget framework, and other aspects of PFM, which the new finance law and related strategic plan supported by significant TA aim to address.

\section{Anchoring fiscal policy with zero} central-bank-financing does not unduly constrain public spending if domestic revenue is increased. The introduction of a value-added tax earlier this year is an important step forward as are recent reforms to tax and customs administration (one-stop customs window, strengthening the capacity of the large taxpayer unit, improving IT systems). However, these measures can be undermined by the lack of concerted effort to close tax loopholes, eliminate exemptions and preferential treatment, raise fuel taxes, and collect more revenue from the natural resource sector and from SOEs. The staff estimates (in the baseline) that more than 1 percent of GDP in additional revenue could be raised this year through implementation of some of these measures. Adopting an automatic fuel pricing mechanism-a stated objective of the authorities-could add even more, while at the same time improve the quality of public spending by eliminating the fiscal cost of the current fuel pricing policy (Annex VII).

\section{Authorities' views}

24. While downplaying concerns about short term fiscal risks, the authorities agreed with the staff's overall analysis and viewed zero financing from the central bank as an important disciplining device in managing public finances. They stressed the importance of IMF TA to help them realize the full potential of the natural resource sector, and they agreed that further domestic revenue mobilization and PFM reforms are crucial for scaling up spending and raising its quality. They appreciated the staff's analysis of fuel pricing and reiterated their intention to reform the current pricing regime. They did not perceive the 2012 budget as a departure from recent practice because it remained tied to the program's fiscal anchor, with a fiscal stance only slightly more expansionary than the staff's baseline.

\section{B. Enhancing the Efficacy of Monetary Policy}

25. Recent weaknesses in the financial and operating performance of the BCC have raised questions from the staff about the direction of its reform. These questions have been far reaching concerning central bank independence, the cost of monetary policy operations, and how to make it more effective. In this regard, a regime change was not seen as appropriate by the staff or the authorities.
26. The efficacy of monetary policy in the DRC is hampered by the high level of dollarization, institutional and administrative weaknesses, and a lack of central bank credibility. Recognizing these constraints discussions centered on the options to strengthen monetary policy and the BCC, with improvements to the status quosupported by TA-being seen as preferable to 
any switch in monetary or exchange rate regime (Annex VIII). In taking this view, the staff understood that improvements to the current framework will take considerable time, including reducing the level of dollarization (Figure 5) and developing the financial market to ensure that monetary policy becomes highly effective and independent (Figure 6). ${ }^{7}$

\section{Several factors favor building on the} status quo. The bipolar view would suggest considering a fixed exchange rate regime, although the staff is not persuaded that the DRC could import credibility this way. A hard peg or full dollarization would require significantly more international reserves to back the local currency than the BCC currently possess, on top of whatever level of reserves the authorities consider necessary to fulfill its lender of last resort function, and the DRC's highly concentrated export base makes full dollarization or a fixed exchange rate a less attractive option. Under the status quo, the DRC maintains the option of a real devaluation to help absorb the external shocks that the economy frequently faces.

\section{To improve on the status quo, the} staff urged the authorities to accelerate the implementation of ongoing reforms. These reforms include recapitalizing the BCC and its restructuring it to align its operations with its core mandate; strengthening the BCC's institutional capacity through better data, information, and analytical capacity; deepening financial markets to enhance the monetary transmission mechanism; and measures to

\footnotetext{
${ }^{7}$ Although growing, the financial sector in the DRC remains small and shallow: broad money as a share of GDP is low by international and sub-regional comparison as is access to banking services.
}

encourage de-dollarization. ${ }^{8}$ These reforms would establish a more flexible and modernized monetary policy framework, anchored by a coherent forward-looking view where monetary aggregates are monitored and analyzed systematically along with a broad array of other macroeconomic and financial indicators, including inflation, output, interest and the exchange rate. Because most of these reforms will take time, the current constraints to an effective and independent monetary policy are likely to remain for some time.

\section{Authorities' views}

29. The authorities welcomed the timely discussion on monetary policy reform options in light of ongoing central bank restructuring, and concurred with the staff's view on the desirability and necessity of strengthening the current monetary and exchange rate regime. While ascribing recent weaknesses in the conduct of monetary policy to lack of coordination with fiscal policy and the financial constraints of the BCC, the authorities stressed the other constraints on the efficacy of monetary policy, especially high dollarization, and they expressed concern about moving further in that direction. In addition to the staff's analysis, they argued that full dollarization would imply a loss of sovereignty and seignorage. The authorities intend to support and safeguard an independent monetary policy regime that also allows for a floating exchange rate, providing an important instrument to mitigate the impact of external shocks.

\footnotetext{
${ }^{8}$ International experience shows that de-dollarization takes years and even decades to reverse, so the DRC authorities should encourage rather than force the issue. Emphasis should, therefore, be placed on market-based measures or so-called pull factors to use local currency.
} 
Figure 5. Foreign Currency Deposits, 2005 and 2010

(Percent of total deposits)

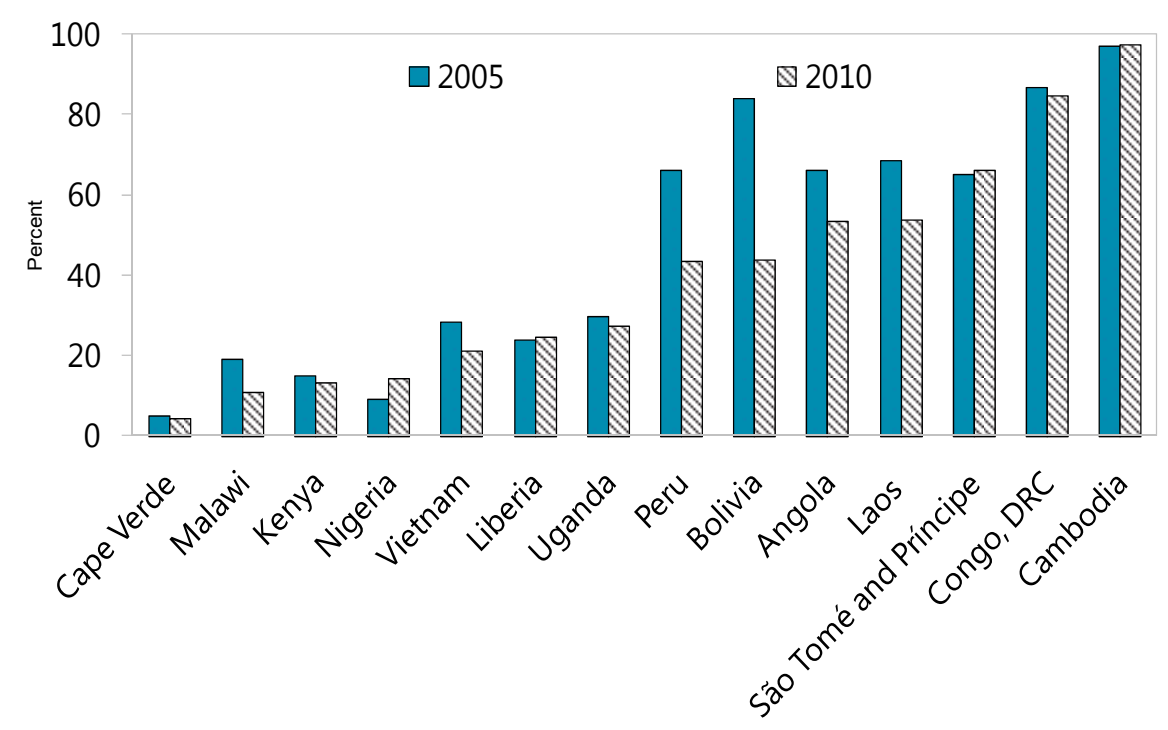

Sources: IMF staff estimates.

Figure 6. Broad Money, 2005 and 2010

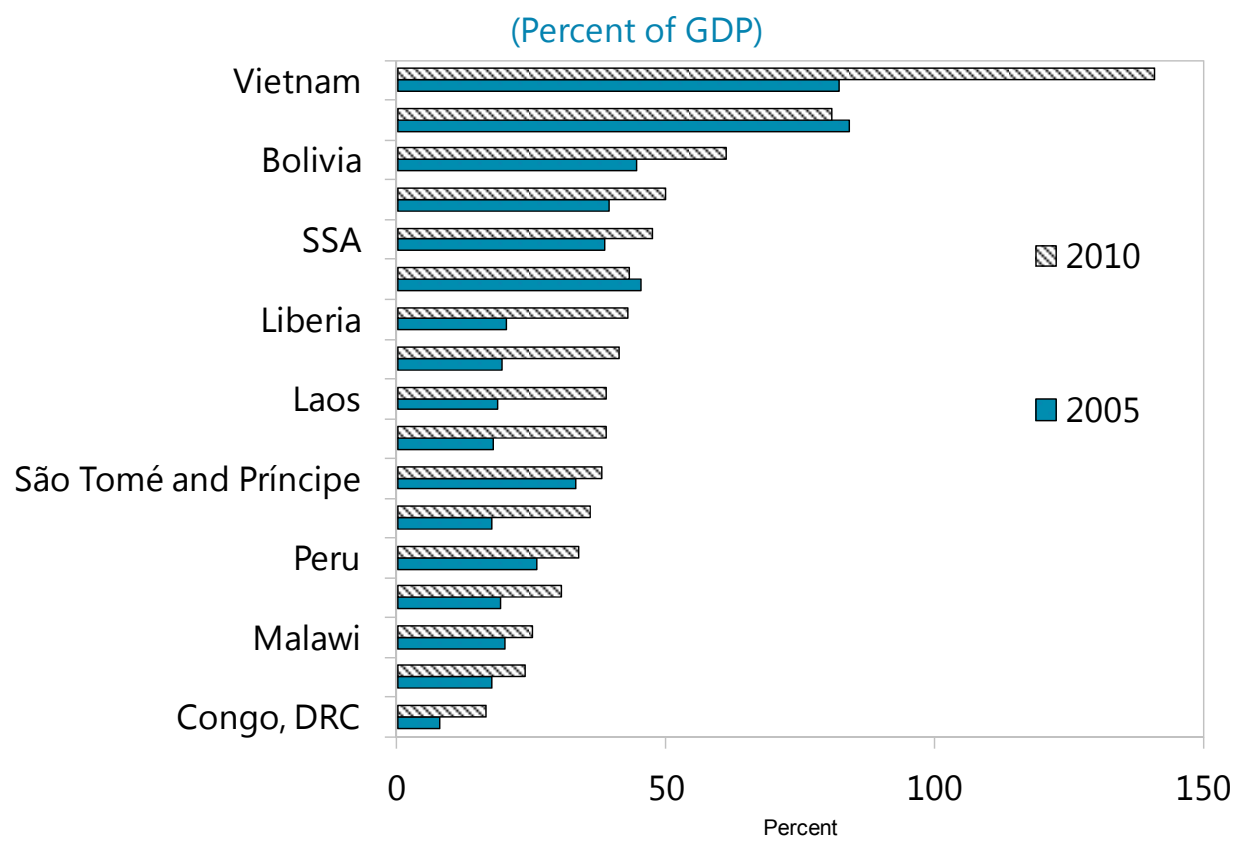

Sources: IMF, World Economic Outlook and International Financial Statistics. 


\section{Making the Economy More Robust}

30. Despite the recent improvement in economic policies and the buildup of the macroeconomic buffers, the DRC economy remains fragile and is vulnerable in two important respects. The financial sector is weak and underdeveloped, limiting growth prospects and the effectiveness of monetary policy; and governance and transparency must be further strengthened if the country is to maximize the benefits of its vast natural resource wealth, or it will risk a slowdown in its development.

\section{Financial Sector Growth and Stability}

\section{Staff noted that DRC's limited} financial integration in the global economy implied that it was largely unaffected from the turmoil in the euro zone. Banks are mostly foreign owned but their financing is mainly domestic, and with a very modest scale of foreign assets and liabilities the direct effects of the turmoil are limited.

\section{More important for the stability of} the financial system is the challenges for banks' profitability posed by the rapid entry of new banks. The 20 commercial banks that are now operating in the DRC do so in a high-cost environment that, even though spreads between deposit and lending rates remain at a relatively high level, squeeze profit margins. ${ }^{9}$ Return on assets at end-2011 was 0.5 percent, which is low compared with banks in neighboring countries, and amongst the

\footnotetext{
${ }^{9}$ The spread between deposit and lending rates in USD has remained around 15 percentage points, while the equivalent in national currency has trended downwards to just under 20 percentage points.
}

lowest in the sub region. Reported financial soundness indicators show a highly capitalized banking system, although compliance with other prudential ratios remains mixed (Table 7).

\section{The soundness of the banking sector} was significantly improved by the closure and liquidation of a systemically important commercial bank in mid-2010. In March 2012 all but two small banks (representing 2 percent of total assets) were in compliance with the required minimum equity, which was raised from US $\$ 5$ million to US $\$ 10$ million in early 2010. All banks now exceed the capital adequacy ratios in terms of risk-weighted assets (33 percent at end-June 2012). The share of non-performing loans (NPLs) of the banking system declined to 8.3 percent of total credits at end-June 2012 from 17 percent at end-2009. Compliance with other prudential ratios remains mixed; four banks, representing a third of the banking system's assets are in violation of the liquidity requirement. The staff advised the authorities to further strengthen data quality, capacity for data assessment, and financial supervision. Pronounced weaknesses in regulatory oversight and prudential enforcement could result in misleading assessments of the state of the financial sector, with adverse consequences for stability.

\section{Staff welcomed the authorities} intention to introduce later this year a new commercial bank and new central bank law developed in line with IMF technical assistance. These laws will help address weaknesses exposed by the closing of the problem bank, including the regulatory and legal framework for liquidation and shareholder rights, bank-resolution 
procedures, and the legal regime for the lender of last resort function. The new laws also aim to strengthen the BCC's ability to coerce troubled banks to address weaknesses in their financial situation and non-compliance with prudential ratios.

\section{The staff also encouraged the} authorities to strengthen their analysis of the risks to financial sector stability from a sharp drop in commodity prices and an abrupt exchange rate adjustment. Because the mining sector is mainly externally financed and banks' exposure to the sector is small, the risks to financial stability from a sharp fall in commodity prices may be limited. However, potential second-round effects from a sharp fall in deposits, deteriorating credit quality, and foreign currency shortage may be substantial. Similarly, an abrupt exchange rate adjustment may have adverse second-round effects on non-collateralized credit in sectors having liabilities in dollars but receiving their revenue in local currency.

\section{Authorities' views}

36. The authorities concurred with the staff's assessment of the challenges and main risks to the financial sector, and asked for the IMF's continued support in identifying risks to strengthening supervision. In particular, they requested an FSAP and would like to pursue the use of stress testing as a tool for assessing vulnerabilities. The authorities also noted their intention to submit to parliament later this year a new payment systems' law that should be instrumental in further reducing transactions costs and developing the banking sector. ${ }^{10}$

\section{Governance and Transparency in Extractive Industries}

37. The implementation of governance and transparency reforms in extractive industries is generally moving forward, in close collaboration with the World Bank and IMF staff. A detailed set of measures is now substantially completed, aiming among other things to ensure the sanctity of contracts and private property rights to enhance the transparency of transactions in these industries. A review of the mining code is also in train, which could address some of the current issues raised by stakeholders. Implementation of a few measures require greater efforts including accession to the New York Convention on the Recognition and Enforcement of Foreign Arbitral Awards; validation by the Extractive Industries Transparency Initiative; and ensuring best practice in the sale of public assets.

38. The recent "commercialization" of a number of SOEs operating in extractive industries, however, has exposed gaps in the policy framework. The transformation from publicly run to commercial enterprises (19 so far) has implied operational

\footnotetext{
${ }^{10}$ The DRC maintains measures that give rise to one exchange restriction and one multiple currency practice subject to IMF approval. The exchange restriction involves an outstanding net debt position against other contracting members under the inoperative regional payments agreement with the Economic Community of the Great Lakes Countries. The multiple currency practice relates to a fixed exchange rate set quarterly applying to transactions through the bilateral payments agreement with Zimbabwe.
} 
independence and resulted in limited accountability to the government (as the sole shareholder). Although the staff of the IMF (and the World Bank) supported this transformation, it is deeply concerned about the weakened accountability to the government and the limited effort these enterprises currently exercise in helping to maximize the wealth of the state, and in generating revenue that should accrue to the Treasury. Equally important is the inability of the government to ensure that SOEs follow and implement national laws regarding investment decisions, contracting external debt, and the sale of public assets.

\section{To rectify this situation, the} government intends to enforce the publication of all transactions in extractive industries, including asset sales on the major SOEs operating in this area (which is mandated by law); call for these SOEs to prepare government-endorsed business plans; review the legal and regulatory framework concerning the governance of these enterprises (with help from the World Bank); establish a mediumterm strategy to address their financial and social debt, and limit their external financing to concessional sources; and require them to set performance targets, for which these

\section{STAFF APPRAISAL}

41. Recent economic performance in the DRC has been surprisingly resilient over the past few years, given weaknesses in the global economy, food and fuel price shocks, domestic security issues, and a protracted election period. Strong ownership in consolidating macroeconomic stability and pursuing economic reform has no doubt enterprises will be held accountable by the government. The objectives of these measures are to (i) ensure the consistent implementation and primacy of the 2008 law guiding the divestiture of state assets; (ii) strengthen and support the enforceability of actions related to transparency and traceability of these transactions, where none currently exists; and (iii) establish clear accountability objectives and compliance rules for the controlling shareholder.

\section{Authorities' views}

40. The authorities confirmed their commitment to the timely publication of contracts in extractive industries, noting that recent delays did not signal any reversal of reforms or commitments in this area. They reiterated their determination to continue with recent efforts and discussions with IMF and World Bank staff, stressing that development of the natural resource sector is fundamental to the DRC's long-term prospects. In doing so, they also noted the difficulties they faced in implementing the laws and regulations governing newly "commercialized" enterprises, but that these problems would be overcome.

helped, and this is reflected in the buildup of the macroeconomic buffers over time.

\section{Notwithstanding better economic} performance and policy implementation, the economy remains vulnerable to external shocks and home-grown risks. This speaks to the importance of further strengthening 
buffers and the institutions and capacity to manage polices to mitigate these events.

\section{Consequently, weaknesses in} formulating the 2012 budget are unfortunate. Until now, fiscal policy had been the foundation for recent performance underpinned by the government's commitment to zero budgetary financing from the central bank, which has helped to bring inflation largely under control. The risks of a softening in the global economy with adverse implications for commodity prices and budgetary revenue, the likelihood of spending overruns needed to address internal security concerns, and lack of foreign financing are cause for deep concern. Fiscal credibility in the DRC has been difficult to earn but it could be easily eroded.

44. To safeguard the fiscal position, the staff urges the government to rigorously monitor budget execution and to reduce non-discretionary spending if it appears the (overly) optimistic revenue projections fail to materialize. At the same time, it must use more effort and make difficult political decisions to raise more domestic revenue, through pricing fuel efficiently and equitably, eliminating ad hoc tax exemptions and loopholes, tightening tax compliance, and ensuring that the natural resource sector contributes its share to the Treasury.

\section{Fiscal risks become even more} worrying in a weak PFM environment. Sustained progress must be made in strengthening the budget process, mediumterm fiscal planning, and the quality of public spending.

46. Similarly, monetary policy must not be derailed. The BCC needs to tightly manage liquidity to dampen inflation expectations and guard against the potential second-round effects from the recent increase in food prices, if it is to achieve its single-digit inflation objective. To do so will also require greater efforts on the part of the authorities to strengthen the BCC's independence, and administrative and institutional capacity. Completing the $\mathrm{BCC}^{\prime}$ s recapitalization, increasing the transparency of its operations, and restructuring it to remove non-core activities are essential.

\section{The current monetary and floating} exchange rate regime remains appropriate to achieve the DRC's broad policy objectives, although a further buildup of international reserves is warranted. The external sector has improved significantly mainly as a result of debt relief but it is vulnerable to external shocks, the narrow export base, and high dollarization, which could tax the monetary authority's ability to act as lender of last resort in the event of a deposit run. If the projected expansion of the mining sector comes to fruition, there may be opportunities to accumulate more international reserves without disrupting the foreign exchange market; and these interventions should be sterilized to offset the impact on the money supply if the inflation target is endangered.

\section{The recent efforts to shore up} financial stability and develop the banking sector are welcomed, especially as they may open opportunities for closer regional and global financial integration. The proposed commercial and central bank regulatory reforms will modernize the legal framework, make the financial system more resilient, and encourage financial deepening. A deepening of the financial market would itself support 
stronger growth, the efficacy of monetary policy, and de-dollarization.

\section{Progress in other structural reforms} has also taken place, notably in public financial management and strengthening governance and transparency in extractive industries, but delays in other areas point to institutional weaknesses. Delays in the implementation of some difficult reforms can be explained by the lack of political leadership and intermittent security concerns during the protracted election period. However, failure to take forward policy changes at a technical level (including some other areas of PFM, revenue mobilization, and restructuring the $\mathrm{BCC}$ ) is unfortunate. While more technical assistance will be required, the political will to build strong institutions is crucial for achieving future reform ambitions and development goals.
50. Enhancing the business environment and establishing a strong foundation for the exploitation and development of DRC's natural resources will be critical. The recent transformation of several SOEs into commercialized entities has created new challenges and highlighted some gaps in the policy framework, which the authorities must address as quickly as possible. Failure to do so may be seen as a reversal of recent progress with unintended consequences for further development in this area.

51. It is proposed that the next Article IV consultation with the DRC be held in accordance with the provisions of the Decision on Article IV Consultation Cycles (Decision No. 14747-(10/96)(9/28/2010). 
Table 1. Democratic Republic of the Congo: Selected Economic and Financial Indicators, 2009-15

\begin{tabular}{|c|c|c|c|c|c|c|c|}
\hline & \multirow[t]{2}{*}{2010} & \multicolumn{2}{|c|}{2011} & \multirow{2}{*}{$\begin{array}{r}2012 \\
\text { Proj } \\
\end{array}$} & \multirow{2}{*}{$\begin{array}{l}2013 \\
\text { Proj } \\
\end{array}$} & \multirow{2}{*}{$\begin{array}{l}2014 \\
\text { Proj } \\
\end{array}$} & \multirow{2}{*}{$\begin{array}{r}2015 \\
\text { Proj } \\
\end{array}$} \\
\hline & & Prog $^{1}$ & Est. & & & & \\
\hline & \multicolumn{7}{|c|}{ (Annual percentage change; unless otherwise indicated) } \\
\hline GDP and prices & & & & & & & \\
\hline Real GDP & 7.2 & 6.5 & 6.9 & 7.2 & 8.2 & 6.4 & 7.3 \\
\hline GDP deflator & 22.1 & 15.0 & 13.4 & 6.6 & 6.7 & 8.7 & 7.6 \\
\hline Consumer prices, period average & 23.5 & 12.0 & 15.5 & 10.9 & 9.5 & 8.8 & 8.3 \\
\hline Consumer prices, end-of-period & 9.8 & 13.0 & 15.4 & 9.9 & 9.0 & 8.5 & 8.0 \\
\hline \multicolumn{8}{|l|}{ External sector } \\
\hline Exports, f.o.b. (U.S. dollars) & 90.4 & 30.9 & 18.1 & -5.6 & 17.2 & 12.8 & 12.6 \\
\hline Imports, f.o.b. (U.S. dollars) & 54.9 & 15.2 & 21.5 & -7.8 & 9.2 & 2.9 & 0.6 \\
\hline Export volume & 47.4 & 7.7 & 21.0 & 8.7 & 19.5 & 10.9 & 12.2 \\
\hline Import volume & 41.7 & 1.6 & 5.4 & -6.2 & 11.6 & 4.2 & 1.3 \\
\hline Terms of trade & 21.7 & 7.2 & -14.1 & -11.6 & 0.2 & 2.9 & 1.1 \\
\hline
\end{tabular}

(Annual change in percent of beginning-of-period broad money; unless otherwise

$$
\text { indicated) }
$$

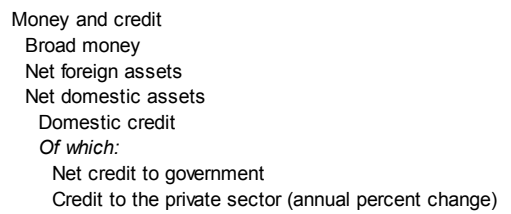

\begin{tabular}{rrrrc}
30.8 & 22.5 & 23.1 & 14.6 & 17.0 \\
63.2 & 9.5 & -3.7 & 10.2 & 8.4 \\
-32.1 & 13.3 & 25.8 & 4.6 & 8.7 \\
-28.9 & 14.3 & 19.1 & 10.3 & 9.9 \\
& & & & \\
-37.5 & 2.4 & 11.6 & 2.8 & 3.3 \\
19.0 & 29.4 & 16.7 & 19.9 & 16.5 \\
\multicolumn{5}{c}{ (Percent of GDP; unless otherwise indicated) }
\end{tabular}

$\begin{array}{rrrrrrr}33.1 & 29.3 & 27.4 & 30.9 & 28.5 & 27.8 & 28.0 \\ 28.1 & 36.9 & 29.1 & 33.5 & 31.8 & 31.5 & 31.4 \\ 0.9 & -1.7 & -1.7 & -0.9 & -0.4 & -0.8 & -0.8\end{array}$

$\begin{array}{lllllll}0.9 & -1.7 & -1.7 & -0.9 & -0.4 & -0.8 & -0.8 \\ & -7.6 & -1.8 & -2.6 & -3.4 & -3.8 & -3.5\end{array}$

$\begin{array}{rrrrrrr}15.5 & 26.8 & 9.0 & 15.7 & 14.5 & 14.2 & 17.7 \\ 7.9 & 7.4 & 4.1 & 7.4 & 8.7 & 10.0 & 10.4 \\ 7.6 & 19.4 & 4.9 & 8.3 & 5.8 & 4.1 & 7.3 \\ 23.6 & 29.6 & 20.5 & 28.2 & 28.7 & 29.5 & 29.7 \\ 8.6 & 13.1 & 6.7 & 9.8 & 9.7 & 8.7 & 8.3 \\ 15.0 & 16.5 & 13.9 & 18.4 & 19.0 & 20.8 & 21.5\end{array}$

(Millions of U.S. dollars; unless otherwise indicated)

$\begin{array}{rcrrrrr}4,571 & 4,939 & 4,732 & 5,595 & 6,466 & 7,357 & 8,077 \\ 3,136 & 3,292 & 3,526 & 4,200 & 4,999 & 5,819 & 6,486 \\ 35.4 & 28.0 & 32.7 & 42.4 & 43.7 & 45.0 & 44.4 \\ 177.0 & 87.5 & 160.0 & 159.6 & 216.2 & 190.6 & 233.9 \\ 2.0 & 0.7 & 1.5 & 1.6 & 1.8 & 1.4 & 1.6 \\ 7.1 & 2.6 & 5.4 & 4.0 & 5.2 & 4.1 & 4.5\end{array}$

Sources: Congolese authorities; and IMF staff estimates and projections.

${ }^{1}$ IMF Country Report No. 11/190.

${ }^{2}$ The projections for 2011 and beyond account for mining companies profit outflows.

${ }^{3}$ Projections are based on calculations under the 2010 HIPC Debt Sustainability Analysis (EBS/10/121, 06/16/2010). Includes assistance beyond the terms of the enhanced HIPC Initiative granted by some Paris Club creditors. Exports are on a three-year backward moving average. 
Table 2. Democratic Republic of the Congo: Balance of Payments, 2010-15

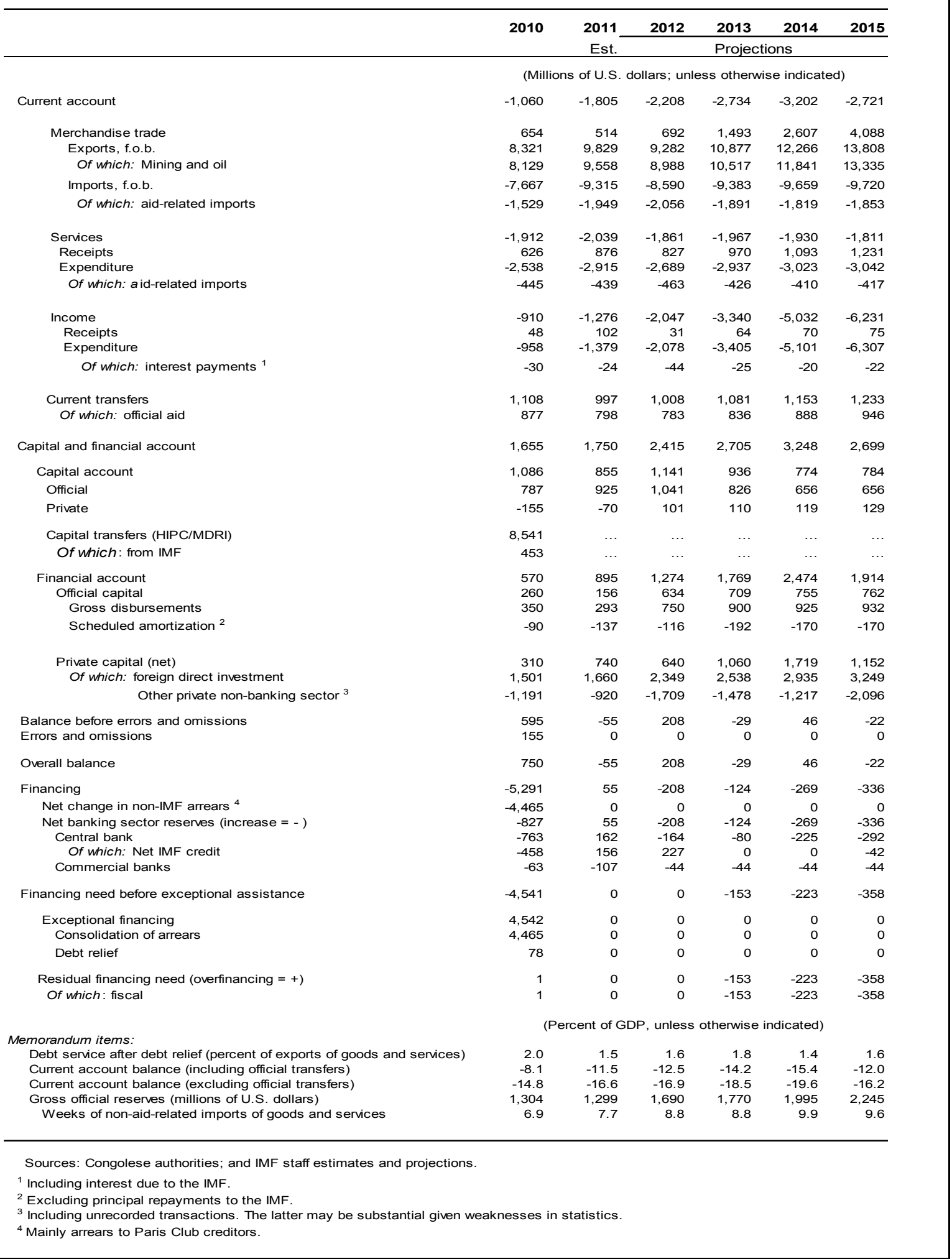


Table 3a. Democratic Republic of the Congo: Central Government Financial Operations, 2010-15

\begin{tabular}{|c|c|c|c|c|c|c|c|}
\hline & \multirow{2}{*}{$\frac{2010}{\text { Est. }}$} & \multicolumn{2}{|c|}{2011} & 2012 & \multirow[t]{2}{*}{2013} & \multirow[t]{2}{*}{2014} & \multirow[t]{2}{*}{2015} \\
\hline & & Prog. ${ }^{1}$ & Est. & Pro & & & \\
\hline \multicolumn{8}{|c|}{ (Billions of Congo francs; unless otherwise indicated) } \\
\hline Revenue & 3,929 & 4,274 & 3,940 & 5,072 & 5,399 & 6,094 & 7,087 \\
\hline Taxes & 1,655 & 2,155 & 2,108 & 2,810 & 3,288 & 3,959 & 4,697 \\
\hline Taxes on income, profits, and capital gains ${ }^{2}$ & 582 & 809 & 708 & 855 & 966 & 1,160 & 1,373 \\
\hline Taxes on goods and services & 742 & 949 & 971 & 1,356 & 1,658 & 2,024 & 2,420 \\
\hline Taxes on international trade & 309 & 396 & 418 & 598 & 664 & 775 & 904 \\
\hline Other taxes & 22 & 0 & 12 & 1 & 0 & 0 & 0 \\
\hline Grants & 1,676 & 1,268 & 1,226 & 1,408 & 1,284 & 1,176 & 1,277 \\
\hline Budget grants & 112 & 46 & 44 & 89 & 0 & 0 & 0 \\
\hline Project grants & 714 & 882 & 849 & 967 & 813 & 690 & 735 \\
\hline HIPC Initiative assistance ${ }^{3}$ & 850 & 340 & 333 & 352 & 471 & 485 & 542 \\
\hline Other revenue & 598 & 852 & 606 & 854 & 828 & 959 & 1,112 \\
\hline Non-tax revenue & 598 & 852 & 606 & 854 & 828 & 959 & 1,112 \\
\hline Of which: Signing bonus for joint venture with China & 0 & 121 & 0 & 117 & 0 & 0 & 0 \\
\hline Expenditure & 3,342 & 5,308 & 4,194 & 5,500 & 6,041 & 6,919 & 7,964 \\
\hline Expense & 2,073 & 2,886 & 2,940 & 3,295 & 3,449 & 3,850 & 4,499 \\
\hline Compensation of employees & 697 & 983 & 1,091 & 1,227 & 1,393 & 1,611 & 1,860 \\
\hline Purchases of goods and services & 607 & 929 & 733 & 1,027 & 1,156 & 1,380 & 1,705 \\
\hline Interest due & 263 & 374 & 381 & 386 & 393 & 408 & 428 \\
\hline Foreign & 235 & 243 & 241 & 256 & 246 & 250 & 260 \\
\hline Domestic & 29 & 131 & 139 & 130 & 147 & 157 & 168 \\
\hline Other expense & 506 & 601 & 735 & 655 & 507 & 450 & 506 \\
\hline Exceptional expense ${ }^{4}$ & 245 & 322 & 561 & 351 & 130 & 21 & 0 \\
\hline Foreign-financed & 42 & 174 & 174 & 139 & 69 & 21 & 0 \\
\hline Domestic-financed & 204 & 149 & 388 & 212 & 60 & 0 & 0 \\
\hline Budget reserve & 0 & 39 & 0 & 40 & 72 & 66 & 76 \\
\hline Net acquisition of nonfinancial assets & 1,269 & 2,421 & 1,253 & 2,205 & 2,593 & 3,069 & 3,465 \\
\hline Foreign-financed & 962 & 1,826 & 876 & 1,474 & 1,629 & 1,644 & 1,781 \\
\hline Domestic-financed & 307 & 596 & 377 & 732 & 964 & 1,425 & 1,684 \\
\hline Government & 54 & 90 & 82 & 132 & 211 & 268 & 309 \\
\hline Provinces & 253 & 506 & 295 & 600 & 753 & 1,157 & 1,375 \\
\hline Net lending/borrowing (overall balance, incl grants) & 587 & $-1,034$ & -254 & -428 & -642 & -825 & -877 \\
\hline Net acquisition of financial assets ("+": increase in assets) & 563 & -121 & -299 & -45 & -114 & -125 & 0 \\
\hline Domestic & 563 & -121 & -299 & -45 & -114 & -125 & 0 \\
\hline Currency and deposits & 563 & -47 & -229 & -3 & -114 & -125 & 0 \\
\hline Equity other than shares (privatization) & 0 & -74 & -7 & 0 & 0 & 0 & 0 \\
\hline Other (e.g., mobile phone license sales) & 0 & 0 & -63 & -42 & 0 & 0 & 0 \\
\hline Foreign & 0 & 0 & 0 & 0 & 0 & 0 & 0 \\
\hline Net incurrence of liabilities ("+": increase in liabilities) & -23 & 913 & -45 & 383 & 378 & 465 & 475 \\
\hline Domestic & -40 & -66 & -56 & -70 & -100 & -100 & -100 \\
\hline Loans & 0 & 0 & 0 & 0 & 0 & 0 & 0 \\
\hline Other accounts payable (arrears) & -40 & -66 & -56 & -70 & -100 & -100 & -100 \\
\hline Foreign & 17 & 979 & 11 & 453 & 478 & 565 & 575 \\
\hline Loans & 17 & 979 & 11 & 453 & 478 & 565 & 575 \\
\hline Amortization (before debt relief) & -298 & -192 & -260 & -247 & -442 & -442 & -504 \\
\hline Debt relief & -2 & 12 & 2 & 2 & 35 & 33 & 34 \\
\hline Project Loans & 317 & 1,158 & 269 & 697 & 886 & 974 & 1,046 \\
\hline Statistical discrepancy & -1 & 0 & 0 & 0 & 151 & 235 & 402 \\
\hline \multicolumn{8}{|l|}{ Memorandum items: } \\
\hline GDP & 11,875 & 14,585 & 14,393 & 16,436 & 18,970 & 21,947 & 25,332 \\
\hline Domestically financed spending & 2,143 & 3,131 & 2,958 & 3,702 & 4,197 & 5,103 & 6,022 \\
\hline Expenditure on education, health, and rural sector development & 699 & 942 & 942 & 1,076 & 1,242 & 1,437 & 1,659 \\
\hline Domestic fiscal balance (cash basis $)^{5}$ & 110 & -245 & -244 & -154 & -81 & -186 & -213 \\
\hline Augmented domestic fiscal balance & & & -280 & & & & \\
\hline Of which: payments by BCC for Banque Congolais liquidation & & & 36 & & & & \\
\hline \multicolumn{8}{|c|}{ Sources: Congolese authorities and IMF staff estimates and projections. } \\
\hline \multirow{4}{*}{\multicolumn{8}{|c|}{$\begin{array}{l}1 \text { IMF Country Report No. 11/190; the ratios are calculated on the basis of revised GDP figures. } \\
2 \text { In } 2011 \text { tax prepayments made by mining firms of } \$ 40 \mathrm{mn} \text {, which will offset profit tax liabilities spread over } 2013-14 . \\
{ }^{3} \text { Reflects revised calculation of HIPC Initiative assistance on the basis of the } 2010 \text { Debt Sustainability Analysis (IMF Country Report No. 10/360). } \\
{ }^{4} \text { Exceptional expense includes spending for the Demobilization, Disarmament, and Reintegration (DDR) program, and cost of the elections. }\end{array}$}} \\
\hline & & & & & & & \\
\hline & & & & & & & \\
\hline & & & & & & & \\
\hline \multicolumn{8}{|c|}{$\begin{array}{l}5 \text { The domestic fiscal balance is defined as revenue (excluding the signing bonus from the SCCA) minus total expenditure (excluding } \\
\text { interest on foreign debt, foreign-financed capital, and exceptional expenditure). }\end{array}$} \\
\hline
\end{tabular}


Table 3b. Democratic Republic of the Congo: Central Government Financial Operations, 2010-15

\begin{tabular}{|c|c|c|c|c|c|c|c|}
\hline & \multirow{2}{*}{$\begin{array}{l}2010 \\
\text { Est. }\end{array}$} & \multicolumn{2}{|c|}{2011} & 2012 & \multirow[t]{2}{*}{2013} & \multirow[t]{2}{*}{2014} & \multirow[t]{2}{*}{2015} \\
\hline & & Prog. $^{1}$ & Est. & Pro & & & \\
\hline \multicolumn{8}{|c|}{ (Billions of Congo francs; unless otherwise indicated) } \\
\hline Revenue & 33.1 & 29.7 & 27.4 & 30.9 & 28.5 & 27.8 & 28.0 \\
\hline Taxes & 13.9 & 15.0 & 14.6 & 17.1 & 17.3 & 18.0 & 18.5 \\
\hline Taxes on income, profits, and capital gains ${ }^{2}$ & 4.9 & 5.6 & 4.9 & 5.2 & 5.1 & 5.3 & 5.4 \\
\hline Taxes on goods and services & 6.2 & 6.6 & 6.7 & 8.2 & 8.7 & 9.2 & 9.6 \\
\hline Taxes on international trade & 2.6 & 2.8 & 2.9 & 3.6 & 3.5 & 3.5 & 3.6 \\
\hline Other taxes & 0.2 & 0.0 & 0.1 & 0.0 & 0.0 & 0.0 & 0.0 \\
\hline Grants & 14.1 & 8.8 & 8.5 & 8.6 & 6.8 & 5.4 & 5.0 \\
\hline Budget grants & 0.9 & 0.3 & 0.3 & 0.5 & 0.0 & 0.0 & 0.0 \\
\hline Project grants & 6.0 & 6.1 & 5.9 & 5.9 & 4.3 & 3.1 & 2.9 \\
\hline HIPC Initiative assistance ${ }^{3}$ & 7.2 & 2.4 & 2.3 & 2.1 & 2.5 & 2.2 & 2.1 \\
\hline Other revenue & 5.0 & 5.9 & 4.2 & 5.2 & 4.4 & 4.4 & 4.4 \\
\hline Non-tax revenue & 5.0 & 5.9 & 4.2 & 5.2 & 4.4 & 4.4 & 4.4 \\
\hline Of which: Signing bonus for joint venture with China & 0.0 & 0.8 & 0.0 & 0.7 & 0.0 & 0.0 & 0.0 \\
\hline Expenditure & 28.1 & 36.9 & 29.1 & 33.5 & 31.8 & 31.5 & 31.4 \\
\hline Expense & 17.5 & 20.1 & 20.4 & 20.0 & 18.2 & 17.5 & 17.8 \\
\hline Compensation of employees & 5.9 & 6.8 & 7.6 & 7.5 & 7.3 & 7.3 & 7.3 \\
\hline Purchases of goods and services & 5.1 & 6.5 & 5.1 & 6.2 & 6.1 & 6.3 & 6.7 \\
\hline Interest due & 2.2 & 2.6 & 2.6 & 2.3 & 2.1 & 1.9 & 1.7 \\
\hline Foreign & 2.0 & 1.7 & 1.7 & 1.6 & 1.3 & 1.1 & 1.0 \\
\hline Domestic & 0.2 & 0.9 & 1.0 & 0.8 & 0.8 & 0.7 & 0.7 \\
\hline Other expense & 4.3 & 4.2 & 5.1 & 4.0 & 2.7 & 2.1 & 2.0 \\
\hline Exceptional expense ${ }^{4}$ & 2.1 & 2.2 & 3.9 & 2.1 & 0.7 & 0.1 & 0.0 \\
\hline Foreign-financed & 0.4 & 1.2 & 1.2 & 0.8 & 0.4 & 0.1 & 0.0 \\
\hline Domestic-financed & 1.7 & 1.0 & 2.7 & 1.3 & 0.3 & 0.0 & 0.0 \\
\hline Budget reserve & 0.0 & 0.3 & 0.0 & 0.2 & 0.4 & 0.3 & 0.3 \\
\hline Net acquisition of nonfinancial assets & 10.7 & 16.8 & 8.7 & 13.4 & 13.7 & 14.0 & 13.7 \\
\hline Foreign-financed & 8.1 & 12.7 & 6.1 & 9.0 & 8.6 & 7.5 & 7.0 \\
\hline Domestic-financed & 2.6 & 4.1 & 2.6 & 4.5 & 5.1 & 6.5 & 6.6 \\
\hline Government & 0.5 & 0.6 & 0.6 & 0.8 & 1.1 & 1.2 & 1.2 \\
\hline Provinces & 2.1 & 3.5 & 2.1 & 3.7 & 4.0 & 5.3 & 5.4 \\
\hline Net lending/borrowing (overall balance, incl grants) & 4.9 & -7.2 & -1.8 & -2.6 & -3.4 & -3.8 & -3.5 \\
\hline Net acquisition of financial assets ("+": increase in assets) & 4.7 & -0.8 & -2.1 & -0.3 & -0.6 & -0.6 & 0.0 \\
\hline Domestic & 4.7 & -0.8 & -2.1 & -0.3 & -0.6 & -0.6 & 0.0 \\
\hline Currency and deposits & 4.7 & -0.3 & -1.6 & 0.0 & -0.6 & -0.6 & 0.0 \\
\hline Equity other than shares (privatization) & 0.0 & -0.5 & 0.0 & 0.0 & 0.0 & 0.0 & 0.0 \\
\hline Other (e.g., mobile phone license sales) & 0.0 & 0.0 & -0.4 & -0.3 & 0.0 & 0.0 & 0.0 \\
\hline Foreign & 0.0 & 0.0 & 0.0 & 0.0 & 0.0 & 0.0 & 0.0 \\
\hline Net incurrence of liabilities ("+": increase in liabilities) & -0.2 & 6.3 & -0.3 & 2.3 & 2.0 & 2.1 & 1.9 \\
\hline Domestic & -0.3 & -0.5 & -0.4 & -0.4 & -0.5 & -0.5 & -0.4 \\
\hline Loans & 0.0 & 0.0 & 0.0 & 0.0 & 0.0 & 0.0 & 0.0 \\
\hline Other accounts payable (arrears) & -0.3 & -0.5 & -0.4 & -0.4 & -0.5 & -0.5 & -0.4 \\
\hline Foreign & 0.1 & 6.8 & 0.1 & 2.8 & 2.5 & 2.6 & 2.3 \\
\hline Loans & 0.1 & 6.8 & 0.1 & 2.8 & 2.5 & 2.6 & 2.3 \\
\hline Amortization (before debt relief) & -2.5 & -1.3 & -1.8 & -1.5 & -2.3 & -2.0 & -2.0 \\
\hline Debt relief & 0.0 & 0.1 & 0.0 & 0.0 & 0.2 & 0.2 & 0.1 \\
\hline Project Loans & 2.7 & 8.0 & 1.9 & 4.2 & 4.7 & 4.4 & 4.1 \\
\hline Statistical discrepancy & 0.0 & 0.0 & 0.0 & 0.0 & 0.8 & 1.1 & 1.6 \\
\hline \multicolumn{8}{|l|}{ Memorandum items: } \\
\hline GDP & 11,875 & 14,585 & 14,393 & 16,436 & 18,970 & 21,947 & 25,332 \\
\hline Domestically financed spending & 18.0 & 21.8 & 20.6 & 22.5 & 22.1 & 23.3 & 23.8 \\
\hline Expenditure on education, health, and rural sector development & 5.9 & 6.5 & 6.5 & 6.5 & 6.5 & 6.5 & 6.5 \\
\hline Domestic fiscal balance (cash basis $)^{5}$ & 0.9 & -1.7 & -1.7 & -0.9 & -0.4 & -0.8 & -0.8 \\
\hline $\begin{array}{l}\text { Augmented domestic fiscal balance } \\
\text { of which: Payments by BCC for Banque Congolais liquidation }\end{array}$ & & & $\begin{array}{r}-1.9 \\
0.2\end{array}$ & & & & \\
\hline
\end{tabular}

Sources: Congolese authorities and IMF staff estimates and projections.

${ }^{1}$ IMF Country Report No. 11/190; the ratios are calculated on the basis of revised GDP figures.

2 In 2011 tax prepayments made by mining firms of \$40mn, which will offset profit tax liabilities spread over 2013-14.

${ }^{3}$ Reflects revised calculation of HIPC Initiative assistance on the basis of the 2010 Debt Sustainability Analysis (IMF Country Report No. 10/360).

${ }^{4}$ Exceptional expense includes spending for the Demobilization, Disarmament, and Reintegration (DDR) program, and cost of the elections.

${ }^{5}$ The domestic fiscal balance is defined as revenue (excluding the signing bonus from the SCCA) minus total expenditure (excluding

interest on foreign debt, foreign-financed capital, and exceptional expenditure). 
Table 4. Democratic Republic of the Congo: Monetary Survey, 2009-13

(Current exchange rates)

\begin{tabular}{|c|c|c|c|c|c|}
\hline & \multirow{2}{*}{$\frac{2009}{\mathrm{Dec}}$} & \multirow{2}{*}{$\begin{array}{r}2010 \\
\mathrm{Dec}\end{array}$} & \multirow{2}{*}{$\frac{2011}{\mathrm{Dec}}$} & 2012 & \multirow{2}{*}{$\begin{array}{c}2013 \\
\text { Dec } \\
\text { Proj. }\end{array}$} \\
\hline & & & & $\begin{array}{l}\text { Dec } \\
\text { Proj. }\end{array}$ & \\
\hline & \multicolumn{3}{|c|}{ (Billions of Congo francs) } & & \\
\hline Net foreign assets & -41.0 & 907.9 & 834.9 & 1082.7 & 1316.8 \\
\hline Net domestic assets & $1,584.5$ & $1,101.7$ & $1,608.5$ & 1721.1 & 1962.0 \\
\hline Domestic credit & 859.4 & 424.9 & 800.7 & 985.2 & 1280.1 \\
\hline Net credit to government & 172.8 & -390.1 & -161.3 & -158.2 & -44.4 \\
\hline Credit to the economy & 686.6 & 815.0 & 962.0 & 1143.4 & 1324.5 \\
\hline Other items, net (including valuation change) & 725.0 & 676.8 & 807.8 & 736.0 & 681.9 \\
\hline Broad money (M2) & $1,501.9$ & $1,964.6$ & $2,418.2$ & 2771.6 & 3241.6 \\
\hline Narrow money (M1) & 479.9 & 706.2 & 789.8 & 938.5 & 1113.9 \\
\hline Currency in circulation & 381.5 & 489.4 & 615.3 & 669.3 & 782.7 \\
\hline Demand deposits & 98.4 & 216.8 & 174.5 & 269.3 & 331.1 \\
\hline Quasi money & $1,022.0$ & $1,258.4$ & $1,628.4$ & 1833.1 & 2127.7 \\
\hline \multirow[t]{2}{*}{ Import deposits } & 41.7 & 45.0 & 25.2 & 32.2 & 37.2 \\
\hline & \multicolumn{4}{|c|}{ (Year-on year change in percent) } & \\
\hline Net foreign assets & 80.6 & $2,316.6$ & -8.0 & 29.7 & 21.6 \\
\hline Net domestic assets & 26.5 & -30.5 & 46.0 & 7.0 & 14.0 \\
\hline Domestic credit & 18.7 & -50.6 & 88.4 & 23.0 & 29.9 \\
\hline Net credit to government & -30.6 & -325.7 & -58.6 & -1.9 & -71.9 \\
\hline Credit to the economy & 44.4 & 18.7 & 18.0 & 18.8 & 15.8 \\
\hline Other items, net (including valuation change) & 37.3 & -6.7 & 19.4 & -8.9 & -7.3 \\
\hline Broad money (M2) & 50.4 & 30.8 & 23.1 & 14.6 & 17.0 \\
\hline Narrow money (M1) & 22.3 & 47.2 & 11.8 & 18.8 & 18.7 \\
\hline \multirow[t]{2}{*}{ Quasi money } & 68.7 & 23.1 & 29.4 & 12.6 & 16.1 \\
\hline & \multicolumn{5}{|c|}{ (Annual change in percent of beginning-of-period broad money) } \\
\hline Net foreign assets & 17.0 & 63.2 & -3.7 & 10.2 & 8.4 \\
\hline Net domestic assets & 33.3 & -32.1 & 25.8 & 4.7 & 8.7 \\
\hline Domestic credit & 13.5 & -28.9 & 19.1 & 7.6 & 10.6 \\
\hline Net credit to government & -7.6 & -37.5 & 11.6 & 0.1 & 4.1 \\
\hline Credit to the economy & 21.2 & 8.5 & 7.5 & 23.0 & 19.3 \\
\hline Other items, net (including valuation change) & 19.7 & -3.2 & 6.7 & -3.0 & -2.0 \\
\hline Broad money (M2) & 50.4 & 30.8 & 23.1 & 14.6 & 17.0 \\
\hline Narrow money (M1) & 8.8 & 15.1 & 4.3 & 6.1 & 6.3 \\
\hline Quasi money & 41.7 & 15.7 & 18.8 & 8.5 & 10.6 \\
\hline \multicolumn{6}{|l|}{ Memorandum items: } \\
\hline Nominal GDP (billions of Congo francs) & 9,073 & 11,875 & 14,393 & 16,436 & 18,970 \\
\hline Velocity (GDP/broad money) & 6.0 & 6.0 & 6.0 & 5.9 & 5.9 \\
\hline Foreign currency deposits (percent of M2) & 67.8 & 63.6 & 66.6 & 65.1 & 64.2 \\
\hline Foreign currency deposits (percent of total deposits) & 90.9 & 84.7 & 89.3 & 85.8 & 84.6 \\
\hline Net foreign assets of the $\mathrm{BCC}^{1}$ & -558 & 172 & 19 & 191 & 288 \\
\hline Net domestic assets of the $\mathrm{BCC}^{1}$ & 1,037 & 451 & 790 & 736 & 796 \\
\hline Of which: Net credit to government from the $\mathrm{BCC}^{1}$ & 200 & -336 & -69 & -66 & 48 \\
\hline Base money ${ }^{1}$ & 479 & 622 & 809 & 927 & 1,084 \\
\hline Of which: currency in circulation ${ }^{1}$ & 395 & 510 & 647 & 757 & 890 \\
\hline \multicolumn{6}{|c|}{ Sources: Congolese authorities; and IMF staff estimates and projections. } \\
\hline${ }^{1}$ In billions of Congolese francs at current exchange $\mathrm{r}$ & & & & & \\
\hline
\end{tabular}


Table 5. Democratic Republic of the Congo: Risk Assessment Matrix

(Scale-high, medium, low)

\begin{tabular}{|c|c|c|}
\hline Source of Risk & Relative Likelihood & Impact if Realized \\
\hline $\begin{array}{l}\text { Strong intensification of the euro } \\
\text { area crisis }\end{array}$ & $\begin{array}{l}\text { Medium } \\
\text { Such intensification could } \\
\text { have adverse implications } \\
\text { for global growth and lead } \\
\text { to a further weakening of } \\
\text { commodity prices }\end{array}$ & $\begin{array}{l}\text { Further deterioration of the current } \\
\text { account deficit if export earnings } \\
\text { decline, other things being equal } \\
\text { Lower direct investment in the } \\
\text { natural resource sector } \\
\text { Slower accumulation of } \\
\text { international reserves }\end{array}$ \\
\hline Fiscal slippages & $\begin{array}{l}\text { High } \\
\text { Unrealistic revenue } \\
\text { projections that are later } \\
\text { unrealized could result in } \\
\text { higher public spending and } \\
\text { an increased deficit } \\
\text { Domestic insecurity in the } \\
\text { eastern region could lead to } \\
\text { higher-than-budgeted } \\
\text { spending }\end{array}$ & $\begin{array}{l}\text { Reversal of recent fiscal } \\
\text { consolidation efforts and return of } \\
\text { fiscal dominance, and inflation } \\
\text { Depreciation of the nominal } \\
\text { exchange rate, with adverse } \\
\text { implications for inflation } \\
\text { Reduced confidence in } \\
\text { macroeconomic policies with } \\
\text { adverse implications for investment }\end{array}$ \\
\hline $\begin{array}{l}\text { Reversal of recent progress in } \\
\text { strengthening governance and } \\
\text { transparency in extractive industries }\end{array}$ & $\begin{array}{l}\text { Low } \\
\text { - Stoppage of all donor } \\
\text { financial support } \\
\text { Deterioration of the business } \\
\text { environment }\end{array}$ & $\begin{array}{l}\text { Need for tightening of the fiscal } \\
\text { position, or central bank financing } \\
\text { of the deficit } \\
\text { Questions about the authorities' } \\
\text { commitment to economic reform } \\
\text { Slowdown in direct investment and } \\
\text { delayed development of the natural } \\
\text { resource sector }\end{array}$ \\
\hline $\begin{array}{l}\text { Steep increase in international food } \\
\text { prices }\end{array}$ & $\begin{array}{l}\text { Medium } \\
\text { International food prices are } \\
\text { on the rise. The effect on the } \\
\text { DRC will depend on the food } \\
\text { crops and composition of } \\
\text { imports }\end{array}$ & $\begin{array}{l}\text { - Increase in headline inflation, } \\
\text { complicating the task of the central } \\
\text { bank } \\
\text { - Slower build-up of reserves }\end{array}$ \\
\hline Steep plunge in commodity prices. & Low (tail risk) & See paragraph 15 for details \\
\hline Regional war in Eastern Congo & Low (tail risk) & See paragraph 15 for details \\
\hline
\end{tabular}


Table 7. Democratic Republic of the Congo: Financial Soundness Indicators, 2003-June 2012

\begin{tabular}{|c|c|c|c|c|c|c|c|c|c|c|}
\hline & 2003 & 2004 & 2005 & 2006 & 2007 & 2008 & 2009 & 2010 & 2011 & Jun-12 \\
\hline \multicolumn{11}{|l|}{ Capital adequacy } \\
\hline Regulatory capital to risk-weighted assets & -3.4 & 6.8 & 7.7 & 10.5 & 12.8 & 10.9 & 17.0 & 29.0 & 30.0 & 33.3 \\
\hline Regulatory Tier I capital to risk-weighted assets & -4.1 & 6.6 & 7.1 & 10.3 & 11.5 & 9.2 & 11.0 & 21.0 & 21.0 & 23.8 \\
\hline \multicolumn{11}{|l|}{ Asset quality } \\
\hline NPLs to gross loans & 1.5 & 2.0 & 6.8 & 3.0 & 4.5 & 2.8 & 7.0 & 7.0 & 6.7 & 6.0 \\
\hline NPLs net of provision to capital & 0.1 & 4.1 & 9.3 & 6.5 & 11.7 & 10.5 & 17.0 & 10.0 & 9.0 & 8.3 \\
\hline \multicolumn{11}{|l|}{ Earnings and profitability } \\
\hline Return on assets & 0.6 & -1.1 & 1.7 & 2.7 & 2.5 & 2.1 & 0.3 & 0.6 & 0.5 & 0.5 \\
\hline Return on equity & 0.6 & -18.5 & 25.3 & 51.6 & 40.8 & 37.6 & 2.5 & 3.0 & 3.0 & 5.4 \\
\hline Interest margin to gross income ${ }^{1}$ & 18.9 & 20.9 & 32.7 & 39.8 & 87.9 & 91.9 & 20.0 & 26.0 & 32.0 & 33.6 \\
\hline Non-interest expenses to gross income & 77.4 & 86.4 & 58.0 & 44.5 & 79.3 & 79.8 & 61.0 & 60.0 & 56.7 & 57.8 \\
\hline \multicolumn{11}{|l|}{ Liquidity } \\
\hline Liquid assets to total assets (liquid asset ratio) & 47.0 & 77.2 & 88.5 & 83.0 & 79.8 & 74.8 & 58.0 & 60.0 & 52.0 & 52.6 \\
\hline Liquid assets to short-term liabilities & 53.6 & 81.4 & 95.0 & 92.9 & 62.9 & 104.0 & 68.0 & 123.0 & 119.0 & 132.2 \\
\hline \multicolumn{11}{|l|}{ Sensibility to market risk } \\
\hline Net open in foreign exchange position to capital & 30.0 & 52.5 & 12.9 & 50.4 & 41.8 & -11.5 & -18.0 & -5.0 & -3.0 & -1.8 \\
\hline $\begin{array}{l}\text { Foreign currency-denominated liabilities to total } \\
\text { liabilities }\end{array}$ & 69.2 & 80.2 & 73.6 & 76.4 & 76.2 & 81.3 & 73.5 & 76.2 & 77.4 & 77.2 \\
\hline \multicolumn{11}{|l|}{ Memoramdum items: } \\
\hline \multicolumn{11}{|l|}{ Capital adequacy } \\
\hline SSA & 13.9 & 15.1 & 15.5 & 16.9 & 16.4 & 17.9 & 19.5 & 19.4 & 20.9 & $\ldots$ \\
\hline Fragile countries & 7.1 & 13.5 & 15.0 & 15.3 & 14.3 & 19.3 & 21.3 & 24.3 & 34.7 & $\ldots$ \\
\hline \multicolumn{11}{|l|}{ Return on assets } \\
\hline SSA & $\ldots$ & $\ldots$ & $\ldots$ & 3.2 & 2.7 & 3.3 & 1.8 & 2.2 & 2.2 & $\ldots$ \\
\hline Fragile countries & $\ldots$ & $\ldots$ & $\ldots$ & 3.7 & 3.2 & 7.0 & 1.1 & 1.7 & 1.0 & $\ldots$ \\
\hline \multicolumn{11}{|l|}{ NPLs to total gross loans } \\
\hline SSA & $\ldots$ & $\ldots$ & $\ldots$ & 12.8 & 10.3 & 8.9 & 9.9 & 9.8 & 8.4 & $\ldots$ \\
\hline Fragile countries & $\ldots$ & $\ldots$ & $\ldots$ & 23.2 & 16.6 & 12.6 & 11.3 & 10.4 & 12.8 & $\ldots$ \\
\hline
\end{tabular}

Source: Central Bank of the Congo (BCC), Directory of Bank Supervision.

Note: Prudential norms were altered throughout the observation period. Application 3 covered 2003 to 2009; application 4, 2010; and application 5; since 2011.

${ }^{1}$ Since 2009 the definition of interest margin to gross income has been made consistent with the IMF reference manuel whereby interest earnings, commissions, and other earnings are reported separately. 


\section{Annex I. Updated Debt Sustainability Analysis}

1. While the macroeconomic fundamentals have improved, the DRC is still considered at high risk of debt distress with weaknesses in debt management adding to vulnerabilities. The authorities reported completing most debt rescheduling agreements with bilateral and commercial creditors in the context of HIPC debt relief, and making good faith efforts to reschedule debts with the remaining creditors on comparable terms.

2. As in the 2010 DSA, strong growth over the medium term is supported by large investments in mining and public infrastructure projects, but with the latest projections for mining production substantially higher than before. Based on developments over the last two years and more detailed information for the mining sector on prospective investment and production, the projections for exports over the medium to long term have been revised upward substantially relative to the previous DSA in June 2010 (e.g., the forecast for exports in 2013 is almost 14 percent higher than in the previous DSA) (TableI.1.).

3. The mining investment for the SinoCongolese (SCCA) joint venture known as Sicomines has been rephased and the expected disbursement of the related public infrastructure loans has also been reprofiled. The period over which the loans are expected to be disbursed has been extended, with the last disbursement now expected in 2019 instead of 2014, and the amount of each disbursement is smaller than initially projected.
4. The rephasing of the disbursements for the SCCA infrastructure loans and the delay in the mining project affect the evolution of the public debt stock and repayments in the baseline. The changes to the disbursement schedule reduce the pace at which the debt stock grows, while the mining project delay pushes back the timing for the repayment of the infrastructure loans, because they will be repaid by the government's share of income from the mining project.

Consequently, although the government has guaranteed the public infrastructure loans, the guarantee on remaining debt can only be called starting in 2034 if the infrastructure loans have not been fully repaid. If it is not called the repayment burden does not directly fall on the budget. According to the feasibility study for the project, the government's share of income from the mining project is (conservatively) forecast to completely pay off the infrastructure loans by 2025 .

\section{External DSA Results}

\section{In the previous DSA the DRC was} rated as at "high risk" of external debt distress; this conclusion is maintained in this updated DSA. This is largely a reflection of its poor CPIA rating and the application of the lowest present value (PV) debt ratio threshold levels. Under the baseline scenario the PV of debt-to-GDP ratio is within a percentage point of the threshold from 201417, with the threshold breached in 2016 
(Figure I.1.). ${ }^{1}$ In the historical scenario, the debt ratios are consistently lower than the baseline. In the medium term this is due primarily to the historical average for the current account deficit (6.7 percent of GDP) being lower than the baseline projection (12.9 percent of GDP average over 2012-17).

\section{The "high risk" rating is justified} despite the improvement of baseline projection for the PV of debt ratios relative to the previous DSA. The previous DSA indicated a larger and more sustained breach of the threshold for the PV of debt-to-GDP ratio over the medium term (Figure I.2.). This stems in part from the revision of the disbursement and repayment profiles for the SCCA public infrastructure loans, as discussed above. Without the inclusion in the public debt stock of the guaranteed public infrastructure loans the baseline would be well below the thresholds (Figure I.3.). However, while these loan guarantees cannot be called until 2034 and are not expected to be called in the current baseline projection, the uncertainty inherent in any mining project, particularly owing to lower-than-expected commodity prices or production and higher-than-expected costs, translates into a risk that the government guarantee may eventually be called (at least in part).

\section{The DRC is vulnerable to adverse} exogenous shocks, especially shocks to commodity prices. The most extreme stress scenario for the PV debt-to-GDP, debt-to-

\footnotetext{
${ }^{1}$ The residuals in the external DSA are consistently large throughout the projection period reflecting weak data, which may not appropriately capture certain flows (e.g., private-sector external borrowing, income outflows in the current account).
}

exports, and debt-to-revenue ratios is an exports shock and in each one the threshold is breached, with the PV debt-to-GDP ratio in the exports shock scenario breaching the threshold by 17 percentage points at its peak and the breach persisting from 2013 until 2024.

\section{Public DSA Results}

\section{The DRC's (estimated) domestic} debt is primarily domestic arrears, most of which were incurred prior to the elections

in 2007. The latest estimate for domestic arrears potentially owed by the government at end 2011 is based on a recent audit conducted by the debt management agency, with the assistance of two audit firms. These include arrears owed to domestic creditors, "social debts" (e.g., wages owed to past government employees), arrears owed to state owned enterprises (SOEs), ${ }^{2}$ arrears owed to suppliers and contractors, and unpaid judgments and awards stemming from judicial decisions. At this juncture, not all of the arrears identified in the initial audit have been certified and many claims on the government may be at least partly offset by liabilities owed to the government. Based on the audit, out of potential domestic liabilities outstanding at the end of 2011 worth US $\$ 1.4$ billion (7.9 percent of 2012 GDP), the debt management unit estimates that approximately US $\$ 900$ million (5.1 percent of 2012 GDP) would be valid after

\footnotetext{
${ }^{2}$ Many SOEs also have outstanding tax arrears or arrears to other SOEs. The government, with the assistance of the World Bank, is in the process of assessing balance sheets of key SOEs to determine what offsetting liabilities can be eliminated and which remain outstanding, including for the external debt liabilities of SOEs.
} 
further assessment; this is the value used for this DSA. The baseline includes the expected extinguishment of all arrears by 2023, with no new domestic arrears or domestic debt incurred between 2012 and 2032.

Consequently, while the PV of total public debt-to-GDP ratio is 4.8 percent of GDP higher in 2012 than that of the PV of external debtto-GDP ratio in the baseline scenario, the difference declines quickly and disappears after 2022 (Figure I.4.). ${ }^{3}$

\section{Although the fixed primary balance} scenario outcome is similar to the baseline, the historical scenario leads to a substantial fall in debt levels. Similar to the 2010 DSA, the PV of the debt-to-GDP ratio declines in the historical scenario and eventually becomes negative after 2023. As in the analysis of the external debt, the debt ratio in the historical scenario is lower than the baseline partly as a result of the average historical real GDP growth rate exceeding the average projected growth rate in the baseline. It is due also to the smaller primary deficit (average of 0.5 percent of GDP in 2002-11) used in the historical scenario projections when compared to the average projected primary deficit in the baseline scenario (average 3.1 percent of GDP over 2012-32). The lower primary deficit results in a lower calculated gross financing need and, given the mechanics of the LIC DSA template, ultimately generates negative values for the nominal debt values for the domestic

\footnotetext{
${ }^{3}$ The government's interest payments on the central bank recapitalization bond issued earlier this year is included in the debt service ratios in Figure I.4. and the tables for total public debt, though the recapitalization bond is a perpetual bond whose principal is not callable, so the bond is not included in the stock of debt.
}

and even foreign currency debt. In other words, on average, if real GDP grows as quickly as it did over the last decade and the government pursues a fiscal policy that generates smaller primary deficits than those forecast under the baseline, it could generate sufficient savings such that the country's net debt (debt minus assets) becomes negative.

\section{The most extreme shock scenario} results in debt ratios that are higher than the baseline, but less severe than in the 2010 DSA. The most extreme shock is the shock with real GDP growth at the historical average minus one standard deviation in 2013 and 2014. This implies annual real GDP growth of 4.3 percent in 2013 and 2014 under the shock scenario, compared to the baseline scenario's real GDP growth projections of 8.2 and 6.4 percent in 2013 and 2014, respectively. Although the debt ratios under the stress scenario are higher than in the baseline scenario, the PV of debt-to-GDP ratio does not exceed 35 percent at any point during the projection period and after 2022 it is below the 30 percent threshold applied in the external debt sustainability analysis.

\section{Conclusion}

\section{While the macroeconomic} fundamentals have improved in the DRC, it is still considered at high risk of debt distress because of its vulnerability to exogenous shocks, especially drops in commodity prices and production. It is therefore important that the DRC continues to take a cautious approach to external borrowing with such borrowing only on highly concessional terms. A significant portion of the debt stock in the DRC is attributable to the public guarantee on the SCCA infrastructure loans and the baseline PV of debt ratios do 
improve substantially if the debt covered by public guarantee is excluded. However, not only is the country still vulnerable to a drop in commodity prices that would reduce growth and drive the PV of debt ratios above their thresholds, but a sizable enough price shock could undermine the economics of the mining project that is supposed to pay off the infrastructure loans and lead to the repayment burden for much or all of those loans falling on the government. ${ }^{4}$ Furthermore, the government's debt management and debt risk analysis capabilities remain weak, which increases the risk that the authorities will accumulate an unsustainable level of external debt. Additional risks stem from debts of state owned enterprises (SOEs) that suffer from even greater problems in debt management capacity than the central government; many have outstanding external debts and are incurring new debts (on a concessional basis), though the scope of any (potential) contingent liabilities is not clear at this time.

\begin{tabular}{|c|c|c|c|c|c|c|c|c|c|}
\hline \multicolumn{10}{|c|}{ Table I.1. DRC: Key Macroeconomic Assumptions, 2010-32 } \\
\hline & 2010 & 2011 & 2012 & 2013 & 2014 & 2015 & 2016 & 2017 & 2018-32 \\
\hline & Act. & Est. & \multicolumn{7}{|c|}{ Proj. } \\
\hline Real GDP (annual percentage change) & 7.2 & 6.9 & 7.2 & 8.2 & 6.4 & 7.3 & 5.1 & 8.0 & 4.1 \\
\hline Inflation rate (GDP deflator, percentage change) & 22.1 & 13.4 & 6.6 & 6.7 & 8.7 & 7.6 & 7.2 & 6.2 & 6.3 \\
\hline $\begin{array}{l}\text { Current account balance (incl. grants, percent of } \\
\text { GDP) }\end{array}$ & -8.1 & -11.5 & -12.5 & -14.2 & -15.4 & -12.0 & -15.9 & -8.5 & -4.4 \\
\hline Overall fiscal balance (incl. grants, percent of GDP) & 4.6 & -2.2 & -3.0 & -3.9 & -4.2 & -3.9 & -2.1 & -2.2 & -2.5 \\
\hline Foreign direct investments (percent of GDP) & 11.5 & 10.6 & 13.3 & 13.2 & 14.1 & 14.4 & 13.6 & 12.1 & 8.2 \\
\hline
\end{tabular}

\footnotetext{
$\overline{4}$ Even without the public guarantee on the SCCA infrastructure loans included in the stock of debt, in an exports shock scenario the PV of debt-to-GDP ratio would still exceed the 30 percent threshold by a sizable margin in 2014-22.
} 
Table I.2. DRC: External Debt Sustainability Framework, Baseline Scenario, 2009-32

(Percent of GDP, unless otherwise indicated)

\begin{tabular}{|c|c|c|c|c|c|c|c|c|c|c|c|c|c|c|c|}
\hline & \multicolumn{3}{|c|}{ Actual } & \multirow{2}{*}{$\begin{array}{l}\text { Historical } \\
\text { Average }\end{array}$} & \multirow{2}{*}{$\begin{array}{l}\text { Standard } \\
\text { Deviation }\end{array}$} & \multicolumn{6}{|c|}{ Projections } & \multirow[b]{2}{*}{$\begin{array}{c}\text { 2012-2017 } \\
\text { Average } \\
\end{array}$} & \multirow[b]{2}{*}{2022} & \multirow[b]{2}{*}{2032} & \multirow[b]{2}{*}{$\begin{array}{c}2018-2032 \\
\text { Average }\end{array}$} \\
\hline & 2009 & 2010 & 2011 & & & 2012 & 2013 & 2014 & 2015 & 2016 & 2017 & & & & \\
\hline External debt (nominal) $1 /$ & 136.3 & 35.9 & 30.5 & & & 34.1 & 37.3 & 39.7 & 40.2 & 39.2 & 38.0 & & 30.3 & 16.4 & \\
\hline Of which: public and publicly guaranteed (PPG) & 136.3 & 35.2 & 29.9 & & & 32.3 & 34.7 & 36.5 & 36.8 & 37.4 & 36.5 & & 29.8 & 16.4 & \\
\hline Change in external debt & 3.3 & -100.5 & -5.4 & & & 36 & 31 & 24 & 0.6 & -1.1 & -12 & & -25 & -0.9 & \\
\hline Identified net debt-creating flows & 6.8 & -24.0 & -5.9 & & & -4.2 & -1.8 & -1.5 & -5.4 & 0.1 & -6.8 & & & -6.2 & \\
\hline Non-interest current account deficit & 5.5 & 7.9 & 10.5 & 3.1 & 6.6 & 12.2 & 14.0 & 15.2 & 11.9 & 15.8 & 8.3 & & 5.3 & 0.4 & 4.3 \\
\hline Deficit in balance of goods and services & 15.7 & 9.6 & 8.9 & & & 6.6 & 2.5 & -3.2 & -10.1 & -5.5 & -14.1 & & -17.1 & -24.2 & \\
\hline Exports & 45.2 & 68.3 & 68.3 & & & 57.2 & 61.4 & 64.1 & 66.6 & 63.9 & 70.8 & & 74.7 & 75.6 & \\
\hline Imports & 60.9 & 77.9 & 77.1 & & & 63.8 & 63.9 & 60.9 & 56.5 & 58.4 & 56.7 & & 57.5 & 51.4 & \\
\hline Net current transfers (negative $=$ inflow) & -11.1 & -6.7 & -5.1 & -6.9 & 2.0 & -4.4 & -4.3 & -4.3 & -4.2 & -4.1 & -4.0 & & -3.7 & -2.9 & -3.5 \\
\hline 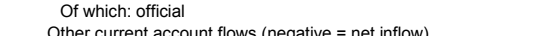 & -11.1 & -6.7 & -5.1 & & & -4.4 & -4.3 & -4.3 & -4.2 & -4.1 & -4.0 & & -3.7 & -2.9 & \\
\hline $\begin{array}{l}\text { Other current account flows (negative = net inflow) } \\
\text { Net FDI (neagative = inflow) }\end{array}$ & $\begin{array}{r}0.9 \\
-102\end{array}$ & $\begin{array}{r}5.0 \\
-11.5\end{array}$ & $\begin{array}{r}6.7 \\
-106\end{array}$ & & & $\begin{array}{r}10.0 \\
133\end{array}$ & $\begin{aligned} 15.9 \\
.13\end{aligned}$ & 22.7 & 26.1 & 25.4 & 26.4 & & 26.2 & 27.5 & \\
\hline $\begin{array}{l}\text { Net FDI (negative }=\text { inflow) } \\
\text { Endogenous debt dynamics } 21\end{array}$ & $\begin{array}{r}-10.2 \\
11.5\end{array}$ & -11.5 & -10.6 & -9.2 & 3.6 & $\begin{array}{r}-13.3 \\
-32\end{array}$ & -13.2 & -14.1 & $\begin{array}{l}-14.4 \\
-2.8\end{array}$ & -13.6 & $\begin{array}{l}-12.1 \\
-2.9\end{array}$ & & -7.6 & -5.8 & -7.3 \\
\hline $\begin{array}{l}\text { Endogenous oebt tynamics } 2 / \\
\text { Contribution from nominal interest rate }\end{array}$ & $\begin{array}{r}11.5 \\
5.7\end{array}$ & $\begin{array}{c}-20.4 \\
0.3\end{array}$ & $\begin{array}{r}-5.8 \\
0.2\end{array}$ & & & $\begin{array}{r}-3.2 \\
0.3\end{array}$ & $\begin{array}{r}-2.6 \\
0.2\end{array}$ & $\begin{array}{r}-2.6 \\
0.2\end{array}$ & $\begin{array}{r}-2.8 \\
0.2\end{array}$ & $\begin{array}{r}-2.1 \\
0.2\end{array}$ & $\begin{array}{r}-2.9 \\
0.2\end{array}$ & & $\begin{aligned}-1.7 \\
0.2\end{aligned}$ & $\begin{array}{r}-0.8 \\
0.1\end{array}$ & \\
\hline Contribution from real GDP growth & $\begin{array}{l}.3 .9 \\
-3.9\end{array}$ & -8.3 & -2.1 & & & -1.9 & -2.6 & -2.2 & -2.7 & -1.9 & $\begin{array}{c}0.2 \\
-2.9\end{array}$ & & -1.3 & $\begin{array}{c}0.1 \\
-0.6\end{array}$ & \\
\hline Contribution from price and exchange rate changes & 9.8 & -12.4 & -3.9 & & & -1.5 & -0.3 & -0.6 & -0.4 & -0.5 & -0.3 & & -0.6 & -0.4 & \\
\hline $\begin{array}{l}\text { Residual (3-4) } 31 \\
\text { Of which: exceptional financing }\end{array}$ & $\begin{array}{l}-3.5 \\
-4.0\end{array}$ & $\begin{array}{l}-76.5 \\
-34.7\end{array}$ & $\begin{array}{l}0.5 \\
0.0\end{array}$ & & & $\begin{array}{l}7.8 \\
0.0\end{array}$ & $\begin{array}{l}4.9 \\
0.0\end{array}$ & $\begin{array}{l}3.9 \\
0.0\end{array}$ & $\begin{array}{l}5.9 \\
0.0\end{array}$ & $\begin{array}{r}-1.1 \\
0.0\end{array}$ & $\begin{array}{l}5.6 \\
0.0\end{array}$ & & $\begin{array}{l}1.5 \\
0.0\end{array}$ & $\begin{array}{l}5.3 \\
0.0\end{array}$ & \\
\hline PV of external debt $4 /$ & $\ldots$ & $\ldots$ & 23.0 & & & 263 & 29.6 & 32.1 & 33.0 & 320 & 31. & & 24.9 & 111 & \\
\hline Percent of exports & & $\ldots$ & 33.6 & & & 46.1 & 48.1 & 50.1 & 49.5 & 50.2 & 43.9 & & 33.3 & 14.7 & \\
\hline PV of PPG external debt & $\ldots$ & ... & 22.3 & & & 24.5 & 27.0 & 28.9 & 29.6 & 30.2 & 29.6 & & 24.4 & 11.1 & \\
\hline Percent of exports & ... & $\ldots$ & 32.7 & & & 42.9 & 43.9 & 45.1 & 44.4 & 47.3 & 41.8 & & 32.7 & 14.7 & \\
\hline Percent of government revenues & & & 118.4 & & & 109.9 & 124.5 & 128.9 & 129.0 & 129.6 & 124.2 & & 97.5 & 43.3 & \\
\hline Debt service-to-exports ratio (percent) & 27.1 & 2.0 & 1.5 & & & 1.6 & 1.9 & 1.5 & 1.7 & 1.9 & 1.7 & & 1.2 & 1.0 & \\
\hline PPG debt service-to-exports ratio (percent) & 27.1 & 2.0 & 1.5 & & & 1.6 & 1.9 & 1.5 & 1.7 & 1.9 & 1.7 & & 1.2 & 1.0 & \\
\hline PPG debt service-to-revenue ratio (percent) & 72.7 & 7.1 & 5.4 & & & 4.2 & 5.3 & 4.4 & 4.9 & 5.2 & 5.0 & & 3.7 & 3.1 & \\
\hline Total gross financing need (Millions of U.S. dollars) & 838.8 & -293.4 & 145.6 & & & -26.0 & 387.2 & 436.8 & -315.6 & 820.4 & -693.9 & & -494.9 & -4276.1 & \\
\hline Non-interest current account deficit that stabilizes debt ratio & 2.2 & 108.3 & 15.9 & & & 8.6 & 10.9 & 12.8 & 11.3 & 16.8 & 9.5 & & 7.8 & 1.3 & \\
\hline \multicolumn{16}{|l|}{ Key macroeconomic assumptions } \\
\hline Real GDP growth (percent) & 2.8 & 7.2 & 6.9 & 5.9 & 1.6 & 7.2 & 8.2 & 6.4 & 7.3 & 5.1 & 8.0 & 7.0 & 4.2 & 3.7 & 4.1 \\
\hline ns (change in percent) & -6.8 & 10.0 & 12.0 & 5.8 & 7.0 & 5.2 & 0.8 & 1.6 & 1.0 & 1.2 & 0.8 & 1.8 & 2.0 & 2.2 & 2.1 \\
\hline Effective interest rate (percent) $5 /$ & 4.1 & 0.2 & 0.5 & 2.8 & 1.7 & 1.1 & 0.6 & 0.6 & 0.7 & 0.6 & 0.7 & 0.7 & 0.8 & 0.9 & 0.9 \\
\hline Growth of exports of G\&S (US dollar terms, percent) & -29.4 & 78.2 & 19.6 & 32.3 & 39.5 & -5.6 & 17.2 & 12.8 & 12.6 & 2.0 & 20.7 & 9.9 & 6.4 & 7.8 & 6.7 \\
\hline Growth of imports of G\&S (US dollar terms, percent) & -23.6 & 50.8 & 18.5 & 31.1 & 30.0 & -6.7 & 9.2 & 2.9 & 0.6 & 9.8 & 5.8 & 3.6 & 5.6 & 4.0 & 5.5 \\
\hline Grant element of new public sector bc & & & & & $\ldots$ & & 31.0 & 31.1 & 31.3 & 32.9 & 33.4 & 31.9 & 44.4 & 41.6 & 41.6 \\
\hline Government revenue (excluding grants, percent of GDP) & 16.8 & 19.0 & 18.9 & & & 22.3 & 21.7 & 22.4 & 22.9 & 23.3 & 23.8 & & 25.0 & 25.6 & 25.2 \\
\hline Aid flows (in Millions of US dollars 71 & 850.1 & 932.31 & 1683.2 & & & 1377.2 & 1347.8 & 1333.7 & 1386.1 & 1089.0 & 1197.1 & & 1301.1 & 3274.8 & \\
\hline Of which: grants & 740.4 & 832.31 & 1233.2 & & & 877.1 & 797.7 & 783.5 & 835.6 & 888.3 & 946.2 & & 1216.6 & 1892.1 & \\
\hline Of which: concessional loans & 109.8 & 100.0 & 449.9 & & & 500.1 & 550.1 & 550.3 & 550.5 & 200.7 & 250.9 & & 84.5 & 1382.7 & \\
\hline Grant-equivalent financing (percent of GDP) $8 /$ & & & $\ldots$ & & & 6.7 & 5.7 & 5.3 & 5.2 & 5.0 & 4.8 & & 4.1 & 3.2 & 3.8 \\
\hline \multirow{2}{*}{\multicolumn{16}{|c|}{ Grant-equivalent financing (percent of external financing) $8 /$}} \\
\hline & & & & & & & & & & & & & & & \\
\hline $\begin{array}{l}\text { Nominal GDPP (Millions of U.S. dollars) } \\
\text { Nominal dollar GDP growth }\end{array}$ & $\begin{array}{l}11,108 \\
-4.2\end{array}$ & 13,093 17.9 & $\begin{array}{l}15,669 \\
19.7\end{array}$ & & & $\begin{array}{l}17,681 \\
12.8\end{array}$ & $\begin{array}{r}19,280 \\
9.0\end{array}$ & $\begin{array}{r}20,841 \\
8.1\end{array}$ & $\begin{array}{r}22,389 \\
8.4\end{array}$ & $\begin{array}{r}24,014 \\
6.3\end{array}$ & $\begin{array}{r}26,142 \\
8.9\end{array}$ & 8.9 & 35,935 6.3 & $\begin{array}{l}64,506 \\
5.9\end{array}$ & 6.2 \\
\hline PV of PPG external debt (in Millions of U.S. dollars) & & & 3,526 & & & 4,243 & 5,029 & 5,829 & 6,485 & 7,067 & 7,555 & & 8598.5 & 7004.1 & \\
\hline (PVt-PVt-1)/GDPt-1 (percent) & & & & & & 4.6 & 4.4 & 4.1 & 3.1 & 2.6 & 2.0 & 3.5 & -0.4 & 0.0 & -0.1 \\
\hline Gross remittances (Millions of U.S. dollars) & 0.0 & 0.0 & 0.0 & & & 0.0 & 0.0 & 0.0 & 0.0 & 0.0 & 0.0 & & 0.0 & 0.0 & \\
\hline PV of PPG external debt (percent of GDP + remittances) & $\ldots$ & $\ldots$ & 22.3 & & & 24.5 & 27.0 & 28.9 & 29.6 & 30.2 & 29.6 & & 24.4 & 11.1 & \\
\hline PV of PPG external debt (percent of exports + remittances) & & $\ldots$ & 32.7 & & & 42.9 & 43.9 & 45.1 & 44.4 & 47.3 & 41.8 & & 32.7 & $\begin{array}{c}14.7 \\
10\end{array}$ & \\
\hline Debt service of PPG external debt (percent of exports + remittances) & & & 1.5 & & & 1.6 & 1.9 & 1.5 & 1.7 & 1.9 & 1.7 & & 1.2 & 1.0 & \\
\hline
\end{tabular}

Sources: Congolese authorities; and IMF staff estimates and projections.

$1 /$ Includes both public and private sector external debt.

Dith $r=$ nominal interest rate; $g=$ real GDP growth rate, and $\rho=$ growth rate of GDP deflator in US. dollar terms

$3 /$ Includes exceptional financing (ie., changes in arrears and debt relief); changes in gross foreign assets; and valuation adjustments. For projections also includes contribution from price and exchange rate changes.

4/ Assumes that PV of private sector debt is equivalent to its face value.

6/ Historical averages and standard deviations are generally derived over the past 10 years, subject to data availability.

8/ Grant-equivalent financing includes grants provided directly to the government and through new borrowing (difference between the face value and the PV of new debt).

CInternational Monetary Fund. Not for Redistribution 


\begin{tabular}{|c|c|c|c|c|c|c|c|c|}
\hline \multicolumn{9}{|c|}{$\begin{array}{l}\text { Table I.3. DRC: Sensitivity Analysis for Key Indicators of Public and Publicly Guaranteed External Debt, } 2012-32 \\
\text { (In percent) }\end{array}$} \\
\hline & \multicolumn{8}{|c|}{ Projections } \\
\hline & 2012 & 2013 & 2014 & 2015 & 2016 & 2017 & 2022 & 2032 \\
\hline & \multicolumn{8}{|c|}{ PV of debt-to GDP ratio } \\
\hline Baseline & 25 & 27 & 29 & 30 & 30 & 30 & 24 & 11 \\
\hline \multicolumn{9}{|l|}{ A. Alternative scenarios } \\
\hline A1. Key variables at their historical averages in 2012-2032 1/ & 25 & 23 & 22 & 23 & 19 & 20 & 11 & 8 \\
\hline A2. New public sector loans on less favorable terms in 2012-2032 2/ & 25 & 22 & 24 & 26 & 28 & 28 & 28 & 34 \\
\hline \multicolumn{9}{|l|}{ B. Bound tests } \\
\hline B1. Real GDP growth at historical average minus one standard deviation in 2013-2014 & 25 & 23 & 26 & 27 & 28 & 28 & 25 & 11 \\
\hline B2. Export value growth at historical average minus one standard deviation in 2013-2014 3/ & 25 & 30 & 46 & 46 & 47 & 46 & 40 & 17 \\
\hline B3. U.S. dollar GDP deflator at historical average minus one standard deviation in 2013-2014 & 25 & 22 & 25 & 27 & 28 & 28 & 25 & 11 \\
\hline B4. Net non-debt-creating flow s at historical average minus one standard deviation in 2013-2014 4/ & 25 & 26 & 33 & 34 & 35 & 35 & 31 & 13 \\
\hline B5. Combination of B1-B4 using one-half standard deviation shocks & 25 & 23 & 24 & 25 & 27 & 27 & 24 & 10 \\
\hline \multirow[t]{2}{*}{ B6. One-time 30 percent nominal depreciation relative to the baseline in 20135 / } & 25 & 31 & 34 & 37 & 38 & 38 & 34 & 15 \\
\hline & \multicolumn{8}{|c|}{ PV of debt-to-exports ratio } \\
\hline Baseline & 43 & 44 & 45 & 44 & 47 & 42 & 33 & 15 \\
\hline \multicolumn{9}{|l|}{ A. Alternative scenarios } \\
\hline A1. Key variables at their historical averages in $2012-20321 /$ & 43 & 38 & 35 & 34 & 30 & 28 & 15 & 10 \\
\hline A2. New public sector loans on less favorable terms in 2012-2032 $2 /$ & 43 & 35 & 38 & 39 & 44 & 40 & 37 & 45 \\
\hline \multicolumn{9}{|l|}{ B. Bound tests } \\
\hline B1. Real GDP growth at historical average minus one standard deviation in 2013-2014 & 43 & 36 & 38 & 39 & 42 & 38 & 32 & 14 \\
\hline B2. Export value growth at historical average minus one standard deviation in 2013-2014 3/ & 43 & 62 & 109 & 107 & 113 & 100 & 83 & 34 \\
\hline B3. U.S. dollar GDP deflator at historical average minus one standard deviation in 2013-2014 & 43 & 36 & 38 & 39 & 42 & 38 & 32 & 14 \\
\hline B4. Net non-debt-creating flow s at historical average minus one standard deviation in $2013-2014$ 4/ & 43 & 43 & 52 & 52 & 55 & 49 & 41 & 17 \\
\hline B5. Combination of B1-B4 using one-half standard deviation shocks & 43 & 36 & 32 & 33 & 36 & 33 & 28 & 12 \\
\hline B6. One-time 30 percent nominal depreciation relative to the baseline in 20135 / & 43 & 36 & 38 & 39 & 42 & 38 & 32 & 14 \\
\hline
\end{tabular}


Table I.3. DRC: Sensitivity Analysis for Key Indicators of Public and Publicly Guaranteed External Debt, 2012-32 (continued) (In percent)

\begin{tabular}{|c|c|c|c|c|c|c|c|c|}
\hline & \multicolumn{8}{|c|}{ Projections } \\
\hline & 2012 & 2013 & 2014 & 2015 & 2016 & 2017 & 2022 & 2032 \\
\hline & \multicolumn{8}{|c|}{ PV of debt-to GDP ratio } \\
\hline Baseline & 25 & 27 & 29 & 30 & 30 & 30 & 24 & 11 \\
\hline \multicolumn{9}{|l|}{ A. Alternative scenarios } \\
\hline A1. Key variables at their historical averages in 2012-2032 1/ & 25 & 23 & 22 & 23 & 19 & 20 & 11 & 8 \\
\hline A2. New public sector loans on less favorable terms in 2012-2032 2/ & 25 & 22 & 24 & 26 & 28 & 28 & 28 & 34 \\
\hline \multicolumn{9}{|l|}{ B. Bound tests } \\
\hline B1. Real GDP growth at historical average minus one standard deviation in 2013-2014 & 25 & 23 & 26 & 27 & 28 & 28 & 25 & 11 \\
\hline B2. Export value growth at historical average minus one standard deviation in 2013-2014 3/ & 25 & 30 & 46 & 46 & 47 & 46 & 40 & 17 \\
\hline B3. U.S. dollar GDP deflator at historical average minus one standard deviation in 2013-2014 & 25 & 22 & 25 & 27 & 28 & 28 & 25 & 11 \\
\hline B4. Net non-debt-creating flows at historical average minus one standard deviation in 2013-2014 4/ & 25 & 26 & 33 & 34 & 35 & 35 & 31 & 13 \\
\hline B5. Combination of B1-B4 using one-half standard deviation shocks & 25 & 23 & 24 & 25 & 27 & 27 & 24 & 10 \\
\hline \multirow[t]{2}{*}{ B6. One-time 30 percent nominal depreciation relative to the baseline in 20135 / } & 25 & 31 & 34 & 37 & 38 & 38 & 34 & 15 \\
\hline & \multicolumn{8}{|c|}{ PV of debt-to-exports ratio } \\
\hline Baseline & 43 & 44 & 45 & 44 & 47 & 42 & 33 & 15 \\
\hline \multicolumn{9}{|l|}{ A. Alternative scenarios } \\
\hline A1. Key variables at their historical averages in 2012-2032 1/ & 43 & 38 & 35 & 34 & 30 & 28 & 15 & 10 \\
\hline A2. New public sector loans on less favorable terms in 2012-2032 $2 /$ & 43 & 35 & 38 & 39 & 44 & 40 & 37 & 45 \\
\hline \multicolumn{9}{|l|}{ B. Bound tests } \\
\hline B1. Real GDP growth at historical average minus one standard deviation in 2013-2014 & 43 & 36 & 38 & 39 & 42 & 38 & 32 & 14 \\
\hline B2. Export value growth at historical average minus one standard deviation in 2013-2014 3/ & 43 & 62 & 109 & 107 & 113 & 100 & 83 & 34 \\
\hline B3. U.S. dollar GDP deflator at historical average minus one standard deviation in 2013-2014 & 43 & 36 & 38 & 39 & 42 & 38 & 32 & 14 \\
\hline B4. Net non-debt-creating flow s at historical average minus one standard deviation in 2013-2014 4/ & 43 & 43 & 52 & 52 & 55 & 49 & 41 & 17 \\
\hline B5. Combination of B1-B4 using one-half standard deviation shocks & 43 & 36 & 32 & 33 & 36 & 33 & 28 & 12 \\
\hline B6. One-time 30 percent nominal depreciation relative to the baseline in 20135 / & 43 & 36 & 38 & 39 & 42 & 38 & 32 & 14 \\
\hline
\end{tabular}


Table I.3. DRC: Sensitivity Analysis for Key Indicators of Public and Publicly Guaranteed External Debt, 2012-32 (concluded) (In percent)

Debt service-to-revenue ratio

\section{Baseline}

\section{A. Alternative scenarios}

A1. Key variables at their historical averages in 2012-2032 1/

A2. New public sector loans on less favorable terms in 2012-2032 2

\section{B. Bound tests}

B1. Real GDP growth at historical average minus one standard deviation in 2013-2014

B2. Export value growth at historical average minus one standard deviation in 2013-2014 3/

B3. U.S. dollar GDP deflator at historical average minus one standard deviation in 2013-2014

B4. Net non-debt-creating flow s at historical average minus one standard deviation in 2013-2014 4/

B5. Combination of B1-B4 using one-half standard deviation shocks

B6. One-time 30 percent nominal depreciation relative to the baseline in 20135 /

Memorandum item:

Grant element assumed on residual financing (i.e., financing required above baseline) 6/

4

4

5

3

3
5

4
6

4

2
7

1
8

$\begin{array}{llllllll}4 & 4 & 5 & 4 & 5 & 5 & \mathbf{4} & \mathbf{3} \\ 4 & 4 & 5 & 5 & 6 & 6 & \mathbf{5} & \mathbf{6} \\ 4 & 4 & 5 & 4 & 5 & 5 & \mathbf{4} & \mathbf{3} \\ 4 & 4 & 5 & 5 & 5 & 5 & \mathbf{4} & \mathbf{4} \\ 4 & 4 & 5 & 4 & 5 & 5 & \mathbf{4} & \mathbf{3} \\ 4 & 6 & 7 & 6 & 6 & 7 & \mathbf{5} & \mathbf{4} \\ & & & & & & & \\ 37 & 37 & 37 & 37 & 37 & 37 & \mathbf{3 7} & \mathbf{3 7}\end{array}$

Sources: Congolese authorities; and IMF staff estimates and projections.

1/ Variables include real GDP growth, growth of GDP deflator (in U.S. dollar terms), non-interest current account in percent of GDP, and non-debt-creating flows

2/ Assumes that the interest rate on new borrowing is by 2 percentage points higher than, but with grace and maturity periods the same as, in the baseline.

3/ Exports values are assumed to remain permanently at the lower level, but the current account as a share of GDP is assumed to return to its baseline level after the shock (implicitly assuming an offsetting adjustment in import levels).

4/ Includes official and private transfers and FDI.

5/ Depreciation is defined as percentage decline in dollar/local currency rate, such that it never exceeds 100 percent.

6/ Applies to all stress scenarios except for A2 (less favorable financing) in which the terms on all new financing are as specified in footnote 2. 
Table I.4. DRC: Public Sector Debt Sustainability Framework, Baseline Scenario, 2009-32

(Percent of GDP, unless otherwise indicated)

\begin{tabular}{|c|c|c|c|c|c|c|c|c|c|c|c|c|c|c|c|}
\hline & \multicolumn{3}{|c|}{ Actual } & \multirow{3}{*}{ Average } & \multirow{3}{*}{$\begin{array}{l}\text { Standard } \\
\text { Deviation }\end{array}$} & \multicolumn{5}{|l|}{ Estimate } & \multicolumn{5}{|c|}{ Projections } \\
\hline & \multirow[b]{2}{*}{2009} & \multirow[b]{2}{*}{2010} & \multirow[b]{2}{*}{2011} & & & \multirow[b]{2}{*}{2012} & \multirow[b]{2}{*}{2013} & \multirow[b]{2}{*}{2014} & \multirow[b]{2}{*}{2015} & \multirow[b]{2}{*}{2016} & \multirow[b]{2}{*}{2017} & \multirow[t]{2}{*}{$\begin{array}{l}2012-17 \\
\text { Average }\end{array}$} & \multirow[b]{2}{*}{2022} & \multirow{2}{*}{\multicolumn{2}{|c|}{2032 Average }} \\
\hline & & & & & & & & & & & & & & & \\
\hline & 146.4 & 42.6 & 35.6 & & & 37.1 & 38.6 & 39.6 & 39.3 & 39.3 & 38.0 & & 29.9 & 16.4 & \\
\hline Of which: foreign-currency denominated & 136.3 & 35.2 & 29.9 & & & 32.3 & 34.7 & 36.5 & 36.8 & 37.4 & 36.5 & & 29.8 & 16.4 & \\
\hline Change in public sector debt & 3.3 & -103.7 & -7.0 & & & 1.5 & 1.5 & 1.0 & -0.3 & 0.0 & -1.3 & & -2.6 & -0.9 & \\
\hline Identified debt-creating flows & -3.2 & -75.4 & -6.6 & & & -2.8 & -1.2 & -0.9 & -1.2 & -0.5 & -1.7 & & -0.7 & 3.1 & \\
\hline Primary deficit & 0.0 & -0.9 & 3.3 & 0.5 & 1.5 & 2.4 & 3.8 & 4.1 & 3.9 & 3.8 & 3.4 & 3.6 & 2.6 & 4.2 & 2.9 \\
\hline Revenue and grants & 20.5 & 25.9 & 25.1 & & & 28.7 & 26.0 & 25.6 & 25.8 & 26.1 & 26.3 & & 26.9 & 25.4 & \\
\hline Of which: grants & 3.7 & 7.0 & 6.2 & & & 6.4 & 4.3 & 3.1 & 2.9 & 2.7 & 2.5 & & 1.8 & 1.0 & \\
\hline Primary (noninterest) expenditure & 20.6 & 25.1 & 28.4 & & & 31.1 & 29.8 & 29.7 & 29.7 & 29.9 & 29.8 & & 29.4 & 29.6 & \\
\hline Automatic debt dynamics & 5.3 & -32.7 & -7.1 & & & -2.8 & -2.5 & -2.8 & -3.0 & -2.2 & -3.1 & & -1.7 & -0.8 & \\
\hline Contribution from interest rate/growth differential & 9.1 & -22.6 & -6.6 & & & -3.6 & -2.9 & -2.8 & -2.9 & -2.1 & -3.0 & & -1.7 & -0.8 & \\
\hline Of which: contribution from average real interest rate & 13.0 & -12.8 & -3.9 & & & -1.2 & -0.1 & -0.4 & -0.2 & -0.2 & -0.1 & & -0.4 & -0.2 & \\
\hline Of which: contribution from real GDP growth & -3.9 & -9.8 & -2.7 & & & -2.4 & -2.8 & -2.3 & -2.7 & -1.9 & -2.9 & & -1.3 & -0.6 & \\
\hline Contribution from real exchange rate depreciation & -3.8 & -10.1 & -0.5 & & & 0.8 & 0.4 & -0.1 & -0.1 & -0.1 & -0.1 & & & & \\
\hline Other identified debt-creating flows & -8.5 & -41.8 & -2.8 & & & -2.4 & -2.5 & -2.2 & -2.1 & -2.1 & -2.0 & & -1.6 & -0.3 & \\
\hline Privatization receipts (negative) & -0.7 & 0.0 & -0.5 & & & -0.3 & 0.0 & 0.0 & 0.0 & 0.0 & 0.0 & & 0.0 & 0.0 & \\
\hline Recognition of implicit or contingent liabilities & 0.0 & 0.0 & 0.0 & & & 0.0 & 0.0 & 0.0 & 0.0 & 0.0 & 0.0 & & 0.0 & 0.0 & \\
\hline Debt relief (HIPC and other) & -7.8 & -41.8 & -2.3 & & & -2.1 & -2.5 & -2.2 & -2.1 & -2.1 & -2.0 & & -1.6 & -0.3 & \\
\hline Other (specify, e.g. bank recapitalization) & 0.0 & 0.0 & 0.0 & & & 0.0 & 0.0 & 0.0 & 0.0 & 0.0 & 0.0 & & 0.0 & 0.0 & \\
\hline Residual, including asset changes & 6.5 & -28.3 & -0.5 & & & 4.3 & 2.7 & 1.9 & 0.9 & 0.5 & 0.3 & & -1.9 & -4.1 & \\
\hline \multicolumn{16}{|l|}{ Other Sustainability Indicators } \\
\hline $\mathrm{PV}$ of public sector debt & 10.0 & 32.9 & 28.0 & & & 29.3 & 30.9 & 32.0 & 32.0 & 32.2 & 31.1 & & 24.5 & 11.1 & \\
\hline Of which: foreign-currency denominated & 0.0 & 25.5 & 22.3 & & & 24.5 & 27.0 & 28.9 & 29.6 & 30.2 & 29.6 & & 24.4 & 11.1 & \\
\hline Of which: external & $\ldots$ & 25.5 & 22.3 & & & 24.5 & 27.0 & 28.9 & 29.6 & 30.2 & 29.6 & & 24.4 & 11.1 & \\
\hline $\mathrm{PV}$ of contingent liabilities (not included in public sector debt) & & & & & & & & & & & & & & & \\
\hline Gross financing need $2 /$ & 10.4 & -4.6 & 3.9 & & & 2.9 & 4.1 & 4.3 & 4.2 & 4.1 & 3.7 & & 2.5 & 4.8 & \\
\hline PV of public sector debt-to-revenue and grants ratio (percent) & 48.8 & 126.8 & 111.8 & & & 102.0 & 118.9 & 125.3 & 124.0 & 123.5 & 118.0 & & 91.1 & 43.6 & \\
\hline PV of public sector debt-to-revenue ratio (percent) & 59.5 & 173.4 & 148.6 & & & 131.4 & 142.4 & 142.8 & 139.7 & 138.0 & 130.4 & & 97.7 & 45.4 & \\
\hline Of which: external $3 /$ & & 134.2 & 118.4 & & & 109.9 & 124.5 & 128.9 & 129.0 & 129.6 & 124.2 & & 97.5 & 45.4 & \\
\hline Debt service-to-revenue and grants ratio (percent) $4 /$ & 52.7 & 11.1 & 8.1 & & & 6.2 & 8.1 & 7.1 & 7.1 & 7.0 & 6.6 & & 4.6 & 3.3 & \\
\hline Debt service-to-revenue ratio (percent) $4 /$ & 76.2 & 19.4 & 11.2 & & & 8.3 & 10.2 & 8.4 & 8.4 & 8.2 & 7.6 & & 5.1 & 3.5 & \\
\hline Primary deficit that stabilizes the debt-to-GDP ratio & -3.3 & 102.8 & 10.4 & & & 0.9 & 2.3 & 3.1 & 4.2 & 3.8 & 4.8 & & 5.2 & 5.2 & \\
\hline Key macroeconomic and fiscal assumptions & & & & & & & & & & & & & & & \\
\hline Real GDP growth (percent) & 2.8 & 7.2 & 6.9 & 5.9 & 1.6 & 7.2 & 8.2 & 6.4 & 7.3 & 5.1 & 8.0 & 7.0 & 4.2 & 3.7 & 4.1 \\
\hline Average nominal interest rate on forex debt (percent) & 4.1 & 0.2 & 0.5 & 2.8 & 1.7 & 1.0 & 0.6 & 0.5 & 0.6 & 0.6 & 0.5 & 0.6 & 0.6 & 0.9 & 0.8 \\
\hline Average real interest rate on domestic debt (percent) & & & -7.0 & $\ldots$ & & -1.9 & -0.9 & -2.9 & -1.9 & -1.6 & -0.7 & & -0.2 & $\ldots$ & $\ldots$ \\
\hline Real exchange rate depreciation (percent, + indicates depreciation) & -2.6 & -8.7 & -1.7 & -0.5 & 14.4 & 2.9 & & & & & & & & - & \\
\hline Inflation rate (GDP deflator, percent) & 35.1 & 22.1 & 13.4 & 19.5 & 8.8 & 6.6 & 6.7 & 8.7 & 7.6 & 7.2 & 6.2 & 7.2 & 6.2 & 6.3 & 6.3 \\
\hline Growth of real primary spending (deflated by GDP deflator, percent) & 0.1 & 0.3 & 0.2 & 0.2 & 0.2 & 0.2 & 0.0 & 0.1 & 0.1 & 0.1 & 0.1 & 0.1 & 0.0 & 0.0 & 0.0 \\
\hline Grant element of new external borrowing (percent) & $\ldots$ & $\ldots$ & $\ldots$ & $\ldots$ & $\ldots$ & & 31.0 & 31.1 & 31.3 & 32.9 & 33.4 & 31.9 & 44.4 & 41.6 & \\
\hline 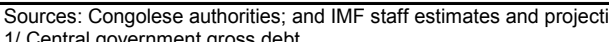 & & & & & & & & & & & & & & & \\
\hline 1/ Central government gross debt. & & & & & & & & & & & & & & & \\
\hline 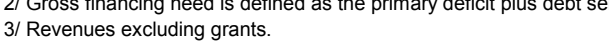 & & ck of & nort-ter & ( & of the & & & & & & & & & & \\
\hline $4 /$ Debt service is defined as the sum of interest and amortization o & I and & ng-term d & & & & & & & & & & & & & \\
\hline 5/ Historical averages and standard deviations are generally derivec & & & & & & & & & & & & & & & \\
\hline
\end{tabular}




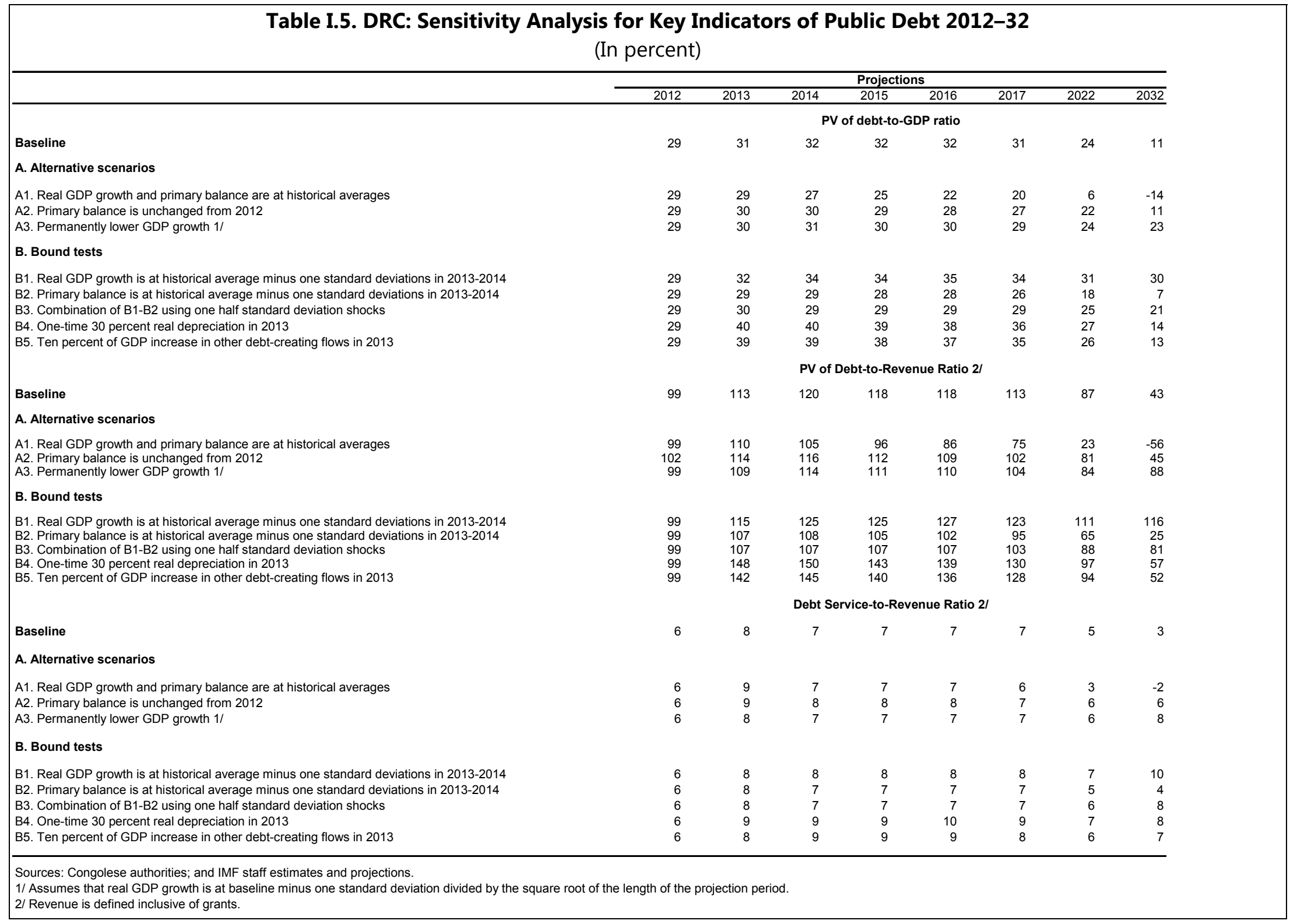


Figure I.1. DRC: Indicators of Public and Publicly Guaranteed External Debt under Alternatives Scenarios, 2012-32 1/
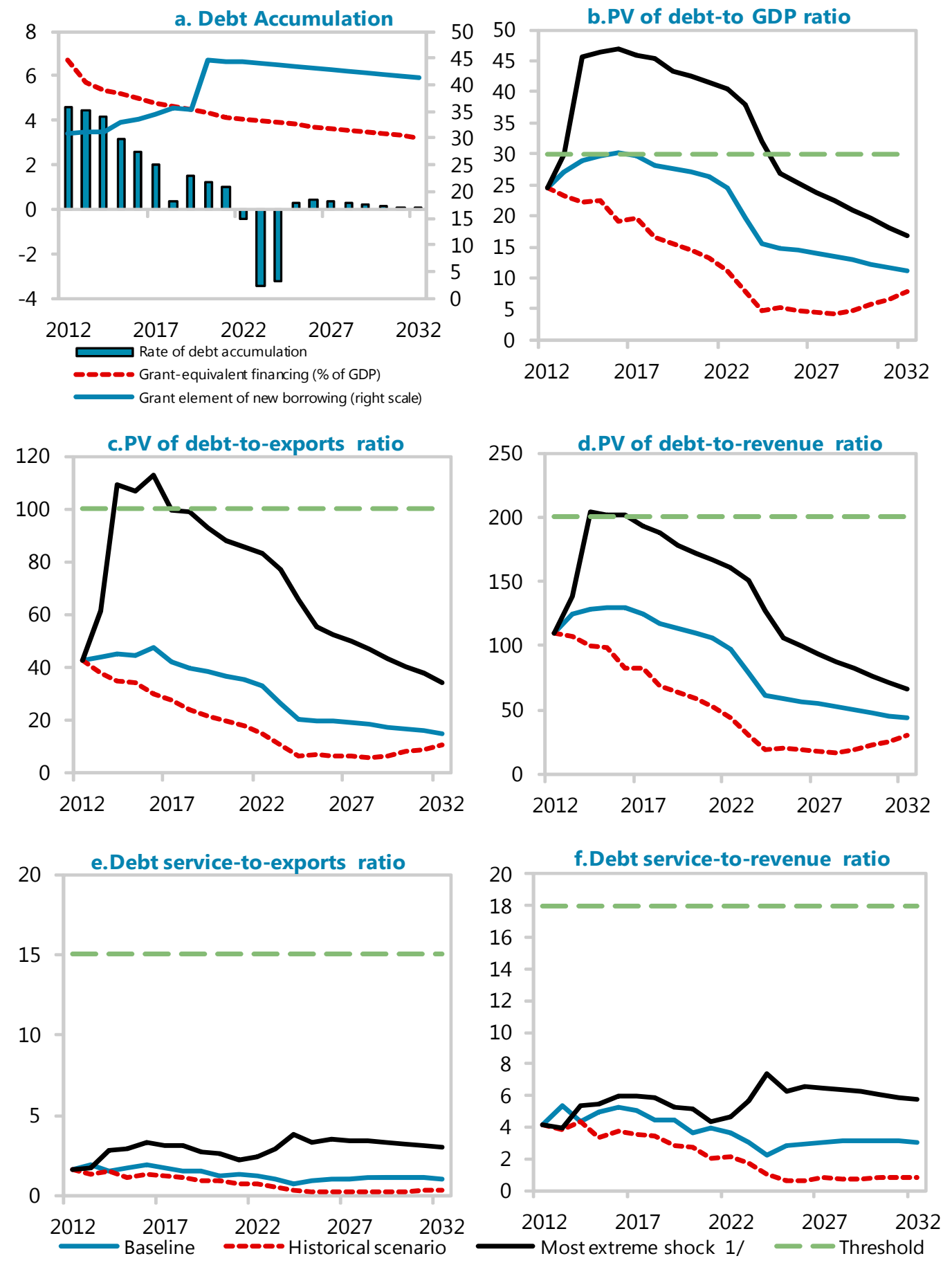

Sources: Congolese authorities; and IMF staff estimates and projections.

$1 /$ The most extreme stress test is the test that yields the highest ratio in 2022. In figure $b$. it corresponds to an Exports shock; in c. to an Exports shock; in d. to an Exports shock; in e. to an Exports shock and in figure f. to a Terms shock 
Figure I.2. DRC: Indicators of Public and Publicly Guaranteed External Debt Baseline Scenarios from 2012 DSA Update and 2010 DSA, 2012-32
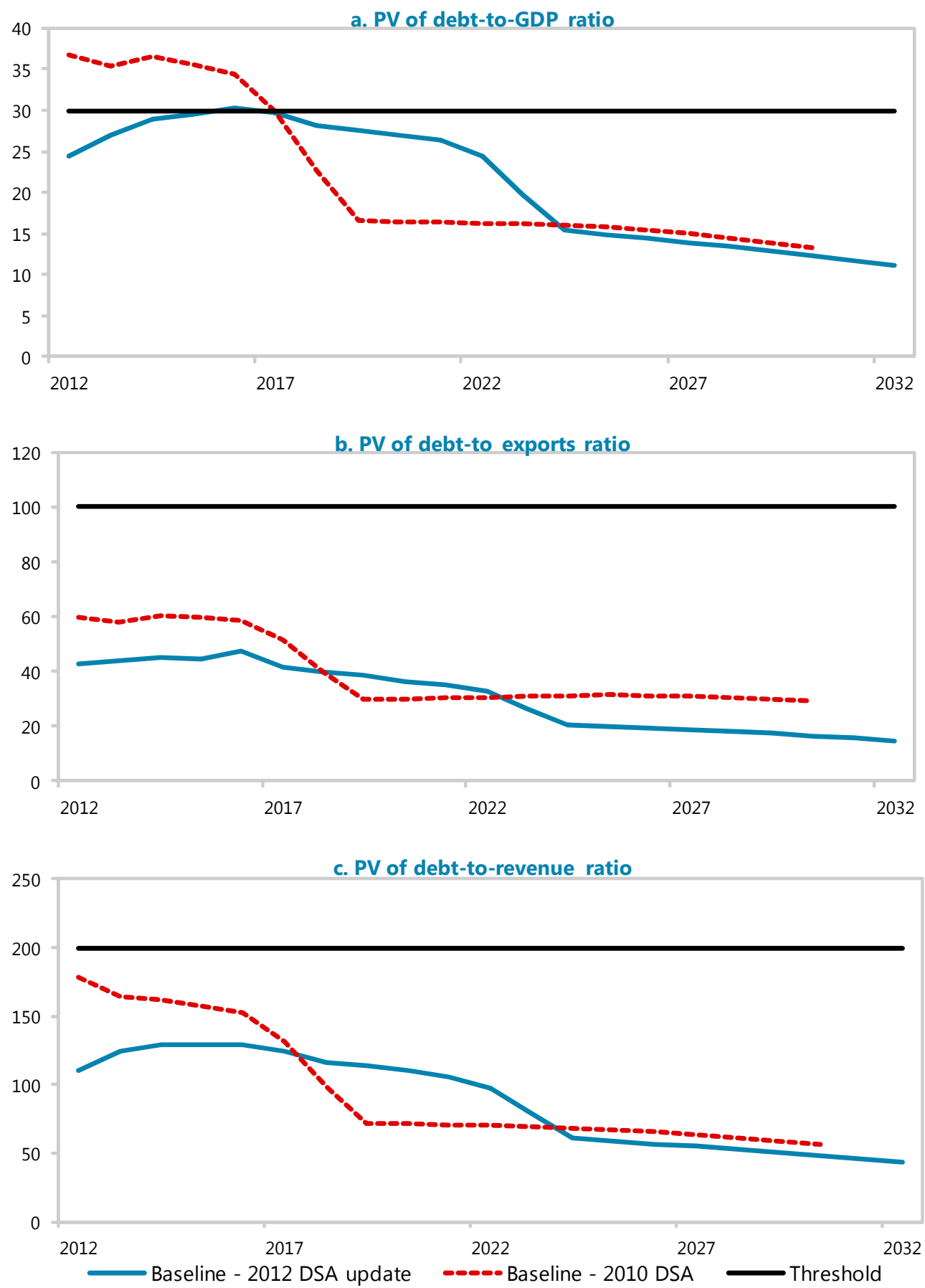

Sources: Congolese authorities; and IMF staff estimates and projections. 
Figure I.3. DRC: Indicators of Public and Publicly Guaranteed External Debt with and Without Public Guarantee on Public Infrastructure
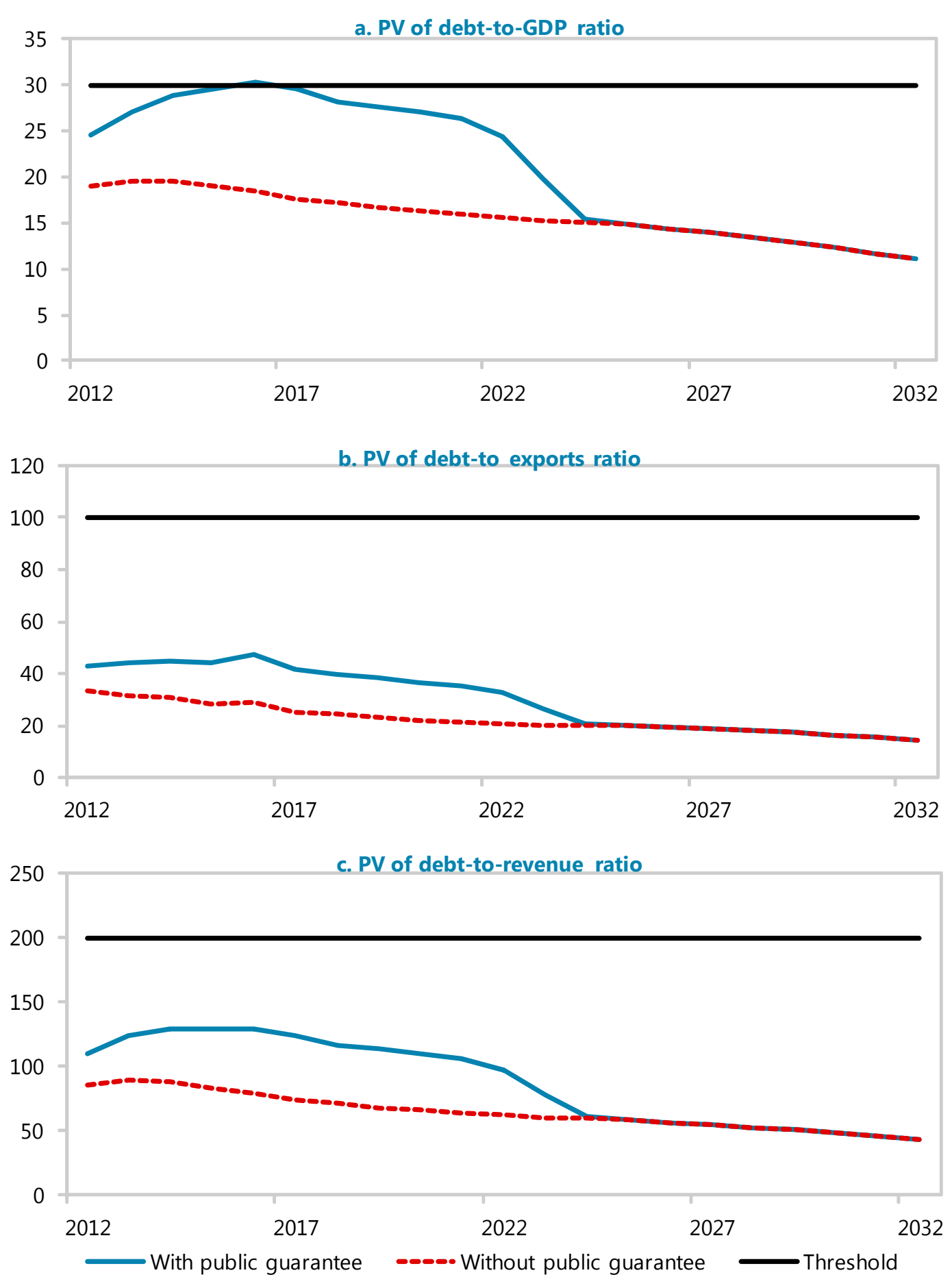

Sources: Congolese authorities; and IMF staff estimates and projections. 
Figure I.4. DRC: Indicators of Public Debt Under Alternatives Scenarios, 2012-32 1/
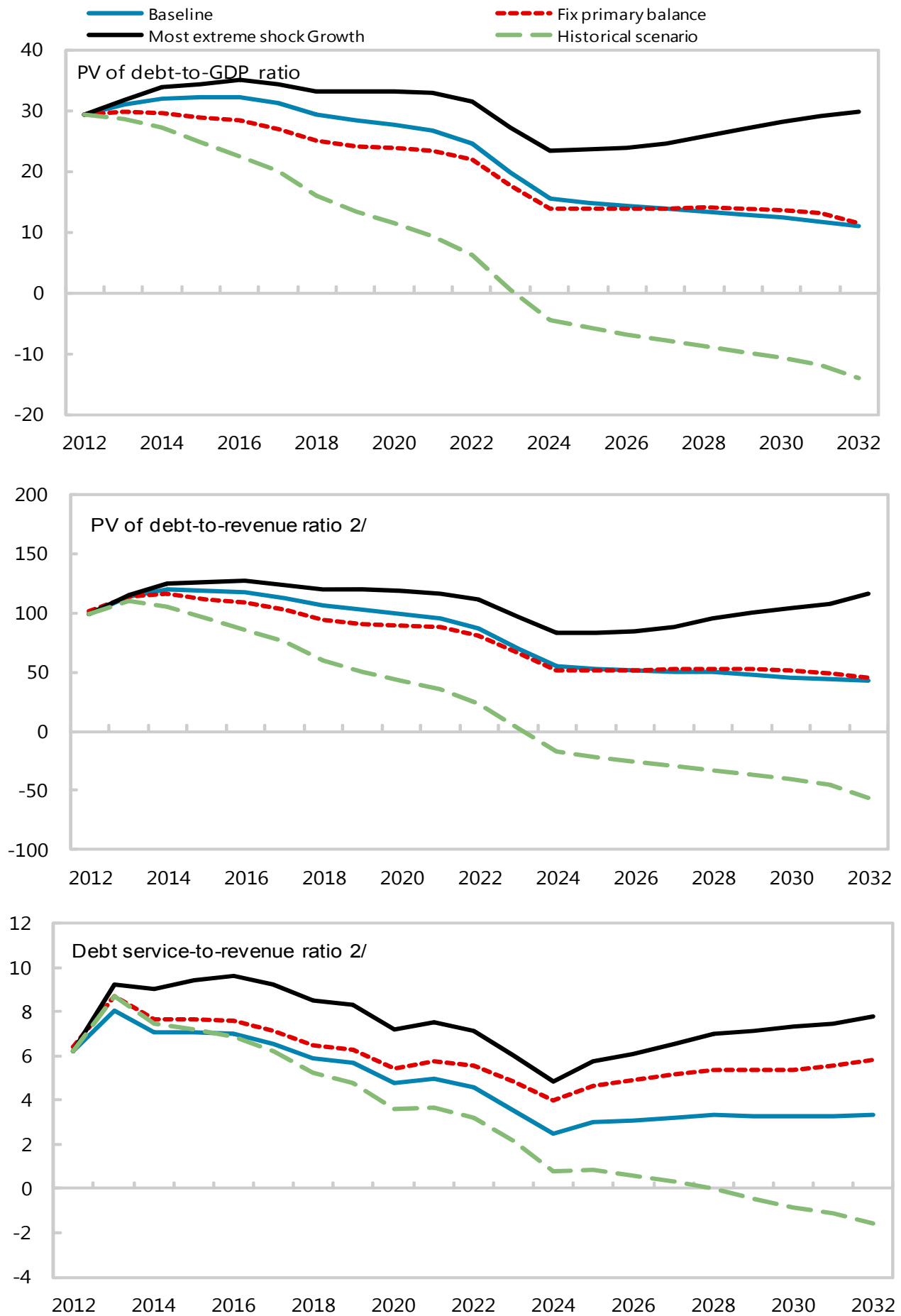

Sources: Congolese authorities; and IMF staff estimates and projections.

$1 /$ The most extreme stress test is the test that yields the highest ratio in 2022.

2/ Revenue is defined inclusive of grants. 


\section{Annex II. Performance Under the Extended Credit Facility Arrangement}

1. In December 2009, the IMF's Executive Board approved a three-year ECF arrangement for the DRC with access of SDR 346.35 million (65 percent of quota).

The third review of the program was completed in April 2011 (IMF Country Report No. 11/190) but since then, program performance has been mixed. The fourth and fifth reviews (based on end-June and endDecember 2011 test dates respectively) could not be completed at this time, due to weaknesses in formulating the 2012 budget and failure to implement structural benchmarks on governance and transparency.

\section{Overall, the authorities have made} good progress in meeting the program's quantitative performance criteria at each of the test dates, especially with respect to the (zero) ceiling on net bank credit to government and the floor on net foreign assets of the BCC. Some external payments arrears accumulated during last year (totaling US\$4.7 million), although all of these arrears were promptly cleared.

3. A sound fiscal position has been the cornerstone of the authorities' program, with its focus on consolidation to reflect limited external financing and the desire to end central bank financing of the budget. The 2012 budget adopted recently by parliament departs from this position, with a resource envelope that is considerably higher than 2011, including a significant increase in budgeted wage payments and overly optimistic revenue projections (roughly

3 percent of GDP above the IMF staff's baseline) not supported by ambitious tax policy or administrative measures. Fully executing such a budget could lead to sizeable fiscal slippages and undermine recent progress, making it difficult to reconcile with the IMF-supported program.

\section{Regarding structural reform, policy} implementation has been mixed. Progress has been made in strengthening aspects of PFM, the independence and operations of the central bank, domestic revenue mobilization, and banking sector regulations. However, the budget process and expenditure execution still exhibit considerable shortcomings, the central bank still faces political pressures that can inhibit monetary policy independence, and efforts to further strengthen governance and transparency in extractive industries (forestry, mining, oil) is lagging in the publication of contracts for the sale of stateowned assets. Better governance and transparency in extractive industries is essential to improve the business climate, maximize the benefits to the country generated by its natural resource wealth, and garner donor support for the DRC's economic development, without which, the objectives of the IMF-supported program may not be achievable. 


\section{Annex III. International Competitiveness}

1. The recent improvement in macroeconomic stability and the fiscal position have had a positive impact on the business climate, although a number of factors still make it a difficult environment to operate in, inhibiting economic development. Factors beyond the level of the real effective exchange rate (REER) play a prominent role.

- While the REER has appreciated over the past several years-largely reflecting terms of trade movements - it is probably not overvalued (Figure III.1). The Consultative Group on Exchange Rate (CGER) methodologies suggest the REER could be about 3-6 percent above its equilibrium value but with wide margins of uncertainty surrounding these estimates - given data limitations for the DRC - it is reasonable to assume it is probably not out of line with the fundamentals.
- The DRC continues to rank near the bottom in cross-country comparisons of the ease of doing business because of administrative complexities (red tape), concerns over governance and corruption, and inefficient and distorted tax policies (Figure III.2). Moreover, the lack of reliable energy supply, transportation networks, social services, and a low level of human capital are significant impediments to developing the non-resource oriented export sector.

- The absence of credit information, a weak judiciary, and poor legal rights seriously limit the capacity of the banking system to provide credit to the economy. In addition, the available credit comes at a relatively high cost and shortterm maturity, further constraining the optimal allocation of resources to medium- to long-term investment projects.

\section{Figure III.1 DRC: Exchange Rates}

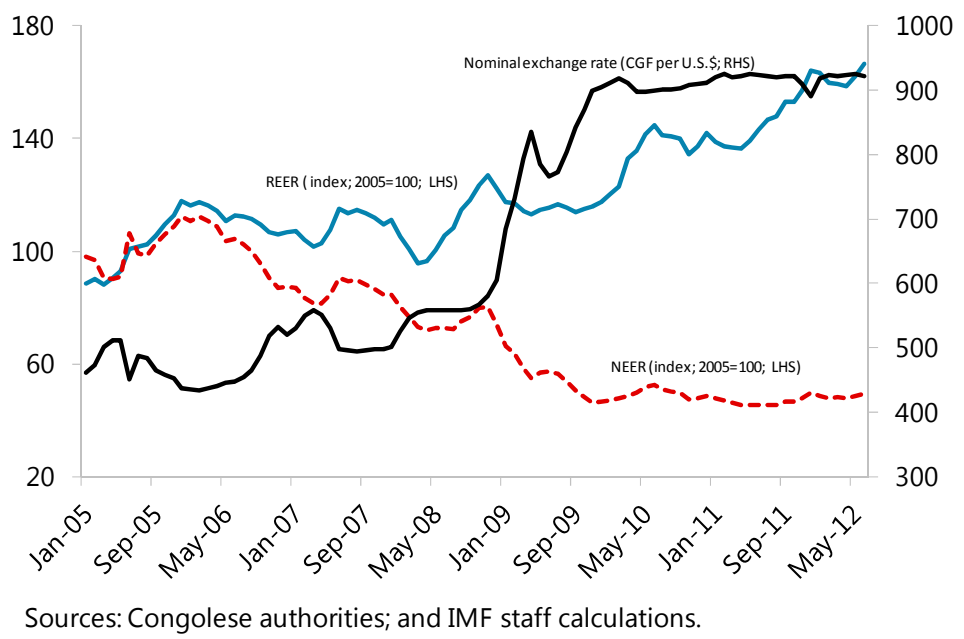




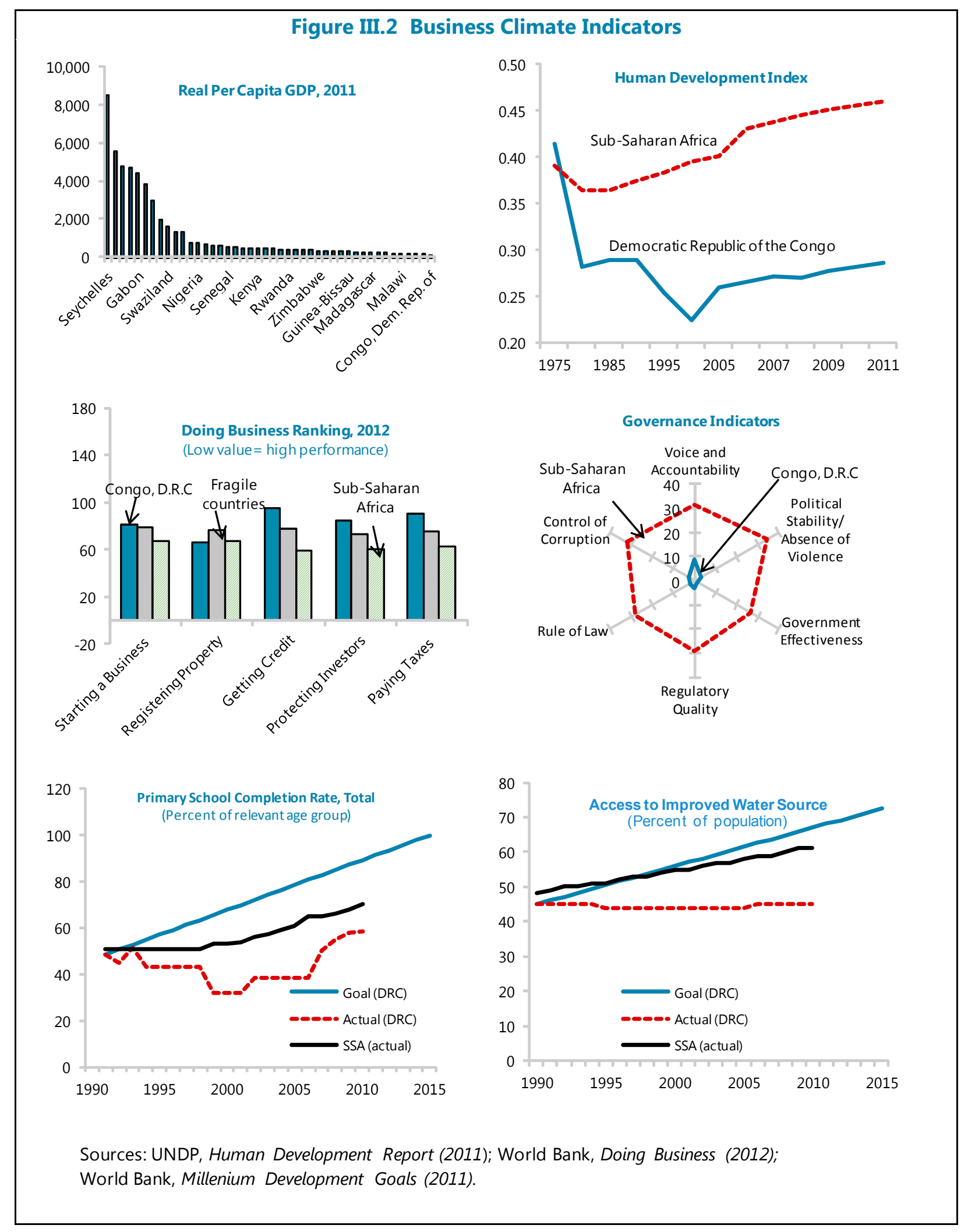




\section{Annex IV. Technical Assistance to the DRC}

1. During the past few years, the DRC has received extensive technical assistance (TA) from the IMF to strengthen its institutional and administrative capacity and will continue to do so in the coming few years.

2. The focus of TA to the central bank and financial sector is on (i) strengthened banking supervision, by means of improved collection and validation of data, and subsequent reporting (including training in on site inspections of banks and financial crisis prevention. i.e., stress testing);

(ii) strengthened internal audit and accounting, notably through the migration of accounts to IFRS; (iii) enhanced capacity for monetary operations and liquidity management through forecasting and improvement of the monetary instruments and their usage; and (iv) improved cash management, through improvements to the infrastructure for the production and distribution of banknotes.

3. These efforts are complemented with TA to enhance the legal framework for the banking sector, notably through support in drafting a new central bank law and a new commercial bank law, to be presented to Parliament later this year.

4. In fiscal policy, the authorities have been moving methodically, if unevenly, forward to put in place their Strategic Plan for Public Finance Reform with TA from the IMF, World Bank, and development partners. Supporting the new finance law and in concert with the strategic plan, significant TA has been provided to address a number of weaknesses in public accounting, expenditure-chain management, the budget framework, and other aspects of PFM. A key element includes efforts to improve the fiscal planning process by moving towards a medium-term budget framework, with expenditure priorities guided by the new PRSP.

\section{A three-year IMF TA program} covering different aspects of the management of the natural resource sector began in the first half of 2012, financed by the Managing Natural Resource Wealth Topical Trust Fund. This program will assist the authorities in improving macrofiscal management and modeling over the medium-term, including revenue administration and tax policy reforms. The initial advice includes establishing an independent macrofiscal forecasting unit, centralizing collection of taxes from extractive industries, simplifying and clarifying the tax code, and developing modeling tools to improve fiscal projections. These tools will also feed into the broader macroeconomic framework and could be used to gain a better understanding of the growth and revenue vulnerabilities the DRC faces because of commodity price volatility.

\section{The IMF is also providing TA to} improve the quality of statistics, in particular national accounts and balance of payment data. 


\section{Annex V. Reserve Adequacy Estimates from the IMF Template}

1. A statistical analysis conducted across sub-Saharan Africa suggests the adverse impact of external shocks is larger in resource exporters than others, and confirms the effectiveness of reserves in preventing and mitigating the effect of these shocks. Ninety-six percent of its exports come from the extractive industries, mainly copper, cobalt, diamonds, gold, and oil. As a large resource exporter, the DRC should hold above-average levels of international reserves.

2. Using the IMF's reserve adequacy template for resource-exporting lowincome countries, the staff estimates that the DRC should hold gross reserves equal to between 10 and 24.7 weeks of non-aidrelated imports, compared with the end2011 level of 7.7 weeks as shown in Figure V.1. This estimate is based on 2012 data and applying an unconditional probability of shocks of 0.5 . The methodology balances the risk-reducing effects of reserves against their carrying cost. It estimates both the likelihood and the scale of a drop in domestic demand in a country and compares the benefits of holding additional reserves in containing declining demand with the costs of holding additional reserves (the potential returns on foregone investment less the returns earned on liquid foreign assets).

\section{In the staff's view, the DRC might} be encouraged to target reserves above the range for two reasons: (i) the price movements of the country's mineral exports are highly correlated thus making terms-oftrade shocks potentially more severe, and (ii) total U.S. dollar deposits in the banking system are currently larger than gross reserves, so the coincidence of a sudden run on the banking system and external shock, would constrain the BCC in its ability to act as a lender of last resort (Figure V.1).

Figure V.1. DRC: Foreign Reserves and Currency Deposits
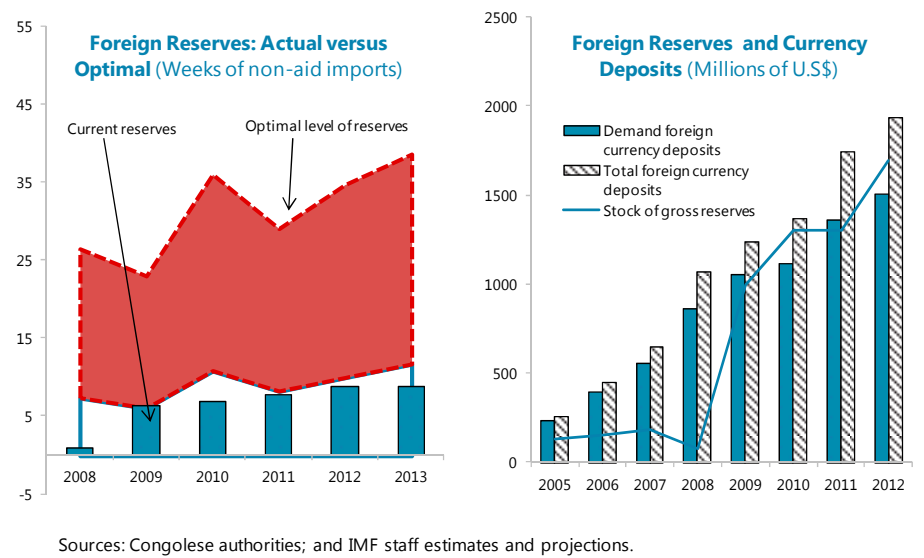


\section{Annex VI. Fiscal Implications of Current Mining-Sector Tax Policies}

1. The Fiscal Analysis of Resource Industries (FARI) model developed by the IMF's Fiscal Affairs Department helps shed light on the fiscal implications of the DRC's tax policies in the mining sector. For a large copper-cobalt project that began in 2006-07 (the project is not identified for reasons of confidentiality), the model demonstrates how these policies result in a non-linear profile for fiscal revenue generated by the project.

2. In the early stage of development, accelerated depreciation allows the company to deduct 60 percent of capital expenditures in the year the investment is made, which typically leads to large losses for tax purposes in the first few years. The firm can then carry forward losses for up to five years that offset taxable income, with the result that it has not paid any profit-based taxes (i.e., corporate income tax, dividend withholding tax, and the state's participation share) up to this point, though it has contributed to the treasury through royalties, customs duties, and payroll taxes.

3. However, beyond the initial development phase, profit-based taxes increase significantly, with the total revenue collected by the state only reaching its peak_possibly as high as US\$900 million a year-a couple of decades after the start of the project. Given that many of the largest mining projects began investing heavily only in the past five years this highly non-linear profile helps to explain why the mining sector's contribution to fiscal revenue in the DRC is relatively low, when compared with its share of GDP.

Figure VI.1. DRC: Fiscal Implications of Current Mining-Sector Tax Policies

(Millions of U.S. dollars)

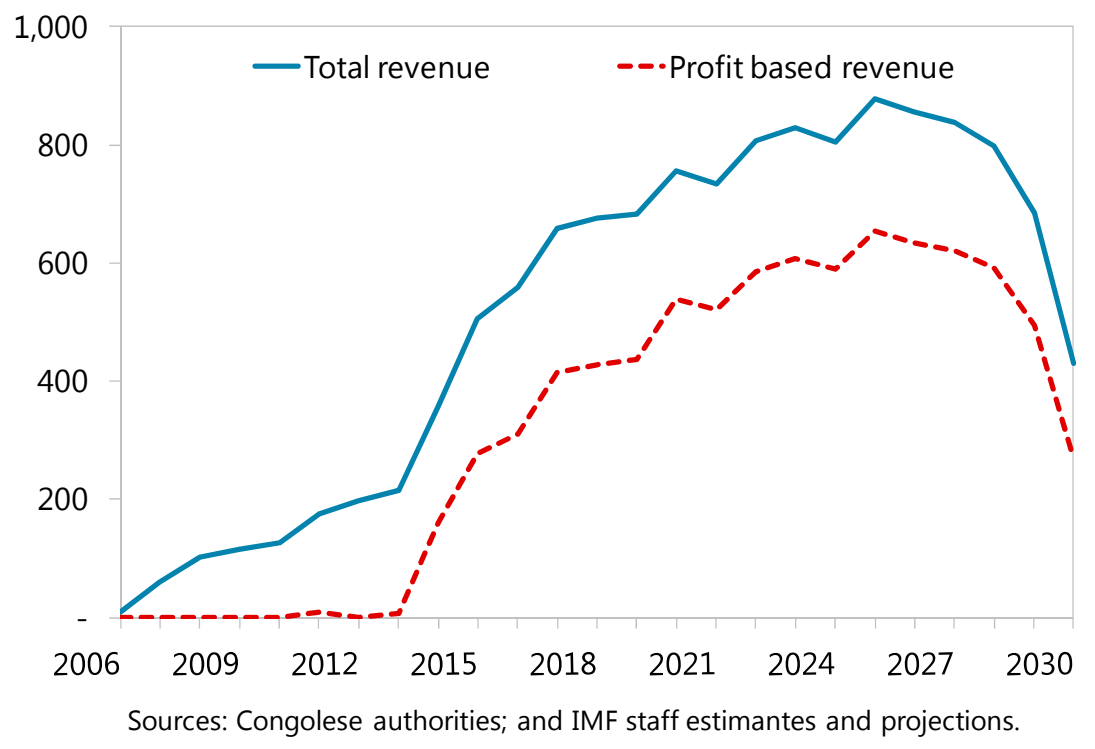




\section{Annex VII. Improving Domestic Fuel Pricing}

1. Under the current fuel pricing regime in the DRC, retail prices of gasoline, diesel, and kerosene are administratively determined and should be adjusted according to a fuel pricing mechanism, but the issue is politicized and adjustments subject to political interference. This results in an ad hoc approach to adjusting fuel prices, leading to volatility in domestic fuel prices and tax revenue. The adoption of an automatic pricing mechanism is intended to reduce this volatility and prevent a sustained decrease in revenue, should international fuel prices continue to increase over a prolonged period.

\section{The current pricing formula} determines retail prices for the main fuel products as the sum of fuel import costs, storage and distribution margins, and net fuel taxes. Less than full pass-through of increases in world oil prices has been achieved by varying the excise tax component, which is derived from the "fiscal price" (or PMFF) rather than the import price (PMFC). ${ }^{1}$ Revenue volatility arising from the application of this price structure has been significant over the past several years. Net taxes, measured as the difference between the retail price and the sum of the CIF import price and margins (including distribution costs), were about 1.1 percent of GDP in 2006 and only 0.6 percent of GDP in 2010, before

\footnotetext{
${ }^{1}$ Under the current law, a rule specifies fuel prices should be adjusted whenever a key variable (i.e., exchange rate, import price, margin costs) changes by more than 5 percent. However, often when the rule requires a price adjustment, there is political resistance and prices are not adjusted according to the rule.
}

rising to 0.8 percent of GDP in 2011 after several retail price increases.

\section{Another way to view the revenue} impact of the current fuel pricing policy is to measure it relative to a (hypothetical) target. For example, we can calculate the target net taxes by setting the target excise rate for gasoline and diesel at 7.5 percent of the import price and for kerosene at 4.5 percent. $^{2}$ We can then estimate the fiscal cost as the difference between the target net taxes and the actual net taxes collected. This implies the fiscal cost of the current pricing regime rose from approximately zero in 2006 to over 0.8 percent of GDP in 2008; a year when the government's total revenue was 18.5 percent of GDP. Although the estimated fiscal cost was lower in 2009 (relative to GDP) as prices were adjusted, it rose again in 2010 and 2011 to roughly the 2008 level (Figure VII.1.).

\section{Automatic Fuel Pricing Mechanisms}

\section{The trade-off between retail price} volatility and fiscal cost is a fundamental concern when designing an automatic fuel pricing mechanism because policymakers

\footnotetext{
${ }^{2}$ Target net taxes $=$ customs duty + sales tax + target excise tax. The target excise tax $=$ statutory rate ${ }^{\star}$ fiscal price (PMFF) and fiscal price $=0.3 *$ import price (PMFC), for effective target excise tax rates of 7.5 (gasoline and diesel) and 4.5 percent (kerosene). Statutory rates for the excise (or consumption) tax are 25 percent of the fiscal price (PMFF) for gasoline and diesel, and 15 percent for kerosene. The target for the PMFF at 30 percent of the PMFC was discussed as a possible structural benchmark in the context of the DRC program, thus it is used here for illustrative purposes.
} 
in many countries attach great importance to shielding poor households from price hikes. Consequently, while an automatic fuel pricing mechanism should ensure full pass through of international price changes over the medium term to limit the fiscal cost of fuel pricing policy and its distortionary effects, it can also be designed to smooth short-term price movements.

5. Agreement on the appropriate level of taxes, distribution margins, and how import price fluctuations are passed through to retail prices is essential for the implementation of an automatic pricing mechanism. Several elements are needed here including specification of the price structure (or formula) for each product, the frequency of parameter adjustments, and a rule that determines the magnitude and timing of retail price changes.

6. Two popular options for short-term price smoothing are moving average (MA, based on the average of past prices) and price band mechanisms (PB, using an upper limit or cap on price adjustments). Regardless of type, any price smoothing mechanism should work symmetrically to be effective and credible. Simulations can provide insights into the inherent trade-offs in the $(2,4$, and 6 month) moving averages and $(3,5$, and 10 percent) price band mechanisms.

7. We compare the simulations of the MA and PB mechanisms to the simulation of the full pass-through case, where there is no short-term price smoothing, and to the DRC's historical fuel pricing policy. The historical series reflects the actual evolution of import prices, margins, and net taxes. The initial margins and taxes for the simulations correspond to the actual price structure in
January 2006. Additionally, the simulations are based on the assumption that margins are adjusted semiannually and that net taxes are adjusted semiannually but not at the same time as the margins, to account for the impact of inflation in eroding the real value of the initial nominal (per liter) tax level.

\section{The simulations show that both} types of smoothing mechanisms can reduce the volatility of domestic retail price changes relative to full pass-through and also prevent the large and sudden price increases experienced under the current approach to fuel pricing. Table VII.1 shows that rankings of volatility measures for retail prices are lower for the price smoothing mechanisms than for the full pass-through and historical (actual) regimes. ${ }^{3}$ Figure VII.2 further illustrates the point that price smoothing mechanisms can reduce retail price volatility relative to full pass-through and the historical fuel pricing policy, especially for temporary sharp increases in import prices: full pass-through in mid-2008 would have resulted in a much higher price level than under a smoothing mechanism. In addition, most smoothing mechanisms also avoid the sharp price increases experienced under the historical regime.

\section{Smoothing mechanisms can also} generate higher, though not necessarily less volatile, tax levels compared with the current fuel pricing policy. Some of these mechanisms can generate relatively low retail-

\footnotetext{
${ }^{3}$ The rankings for each individual volatility measure (e.g., standard deviation of prices or taxes) are relative to the other mechanisms and the overall ranking is based on the sum of rankings across volatility measures.
} 
price volatility but at a cost of higher tax volatility. For example, the 3 percent price band (PB3) and 6-month moving average (MA6) mechanisms are the best two for minimizing price volatility, but the worst two for minimizing tax volatility (Table VII.1). However, more volatility does not necessarily come at the expense of lower revenue: the simulations indicate that the net tax per liter from smoothing mechanisms is higher than that of the historical realizations in almost every month of the sample period (Figure VII.3).

\section{When considering the most} appropriate smoothing mechanism, it is important to balance price and tax volatility concerns. Table VII.1 presents the price and tax volatility rankings of each mechanism and an overall ranking of the mechanisms (see footnote 5). The 3 percent $\mathrm{PB}$ mechanism scores the best overall rank while the historical realizations of the current policy score the worst. Notably, the full-passthrough mechanism is also demonstrably superior to the status quo.

\section{Relative to the current policy and} given the assumptions for generating the simulations, the PB3 mechanism substantially reduces retail price volatility (one of the government's stated priorities), and has a lower fiscal cost during the sample period (Table VII.2). ${ }^{4}$ Over 2006-11,

\footnotetext{
${ }^{4}$ The monthly fiscal cost per liter of each mechanism is calculated as the difference between the monthly net tax level for the mechanism and the full passthrough net tax level. The fiscal cost for the historical series therefore depends in part on the margin and net tax target assumptions made for the full passthrough simulation. These assumptions differ from those used to estimate the fiscal cost by fuel type shown in Figure VII.1.
}

the total fiscal cost of the current policy exceeds 2.8 percent of GDP, whereas the PB3 mechanism's total fiscal cost is less than 0.8 percent of GDP, meaning revenue under the current policy is 200 billion CGF lower than it would have been if the government had employed the PB3 mechanism in $2006-11 .^{5}$

\footnotetext{
${ }^{5}$ While we do not explicitly consider reform strategies for the DRC here, Gupta and others (2000) and Coady and others (2010) discuss price subsidy reform strategies in more detail. Coady and others (2011) also discusses in greater detail the specific steps and institutional arrangements suggested to ensure a successful fuel pricing reform, while also providing specific examples of measures to mitigate the impact on the poor that have been adopted during subsidy reforms in different countries.
} 
Figure VII.1. DRC: Fiscal Cost and Contribution by Fuel Types, 2006-11

\section{(Percent of GDP)}

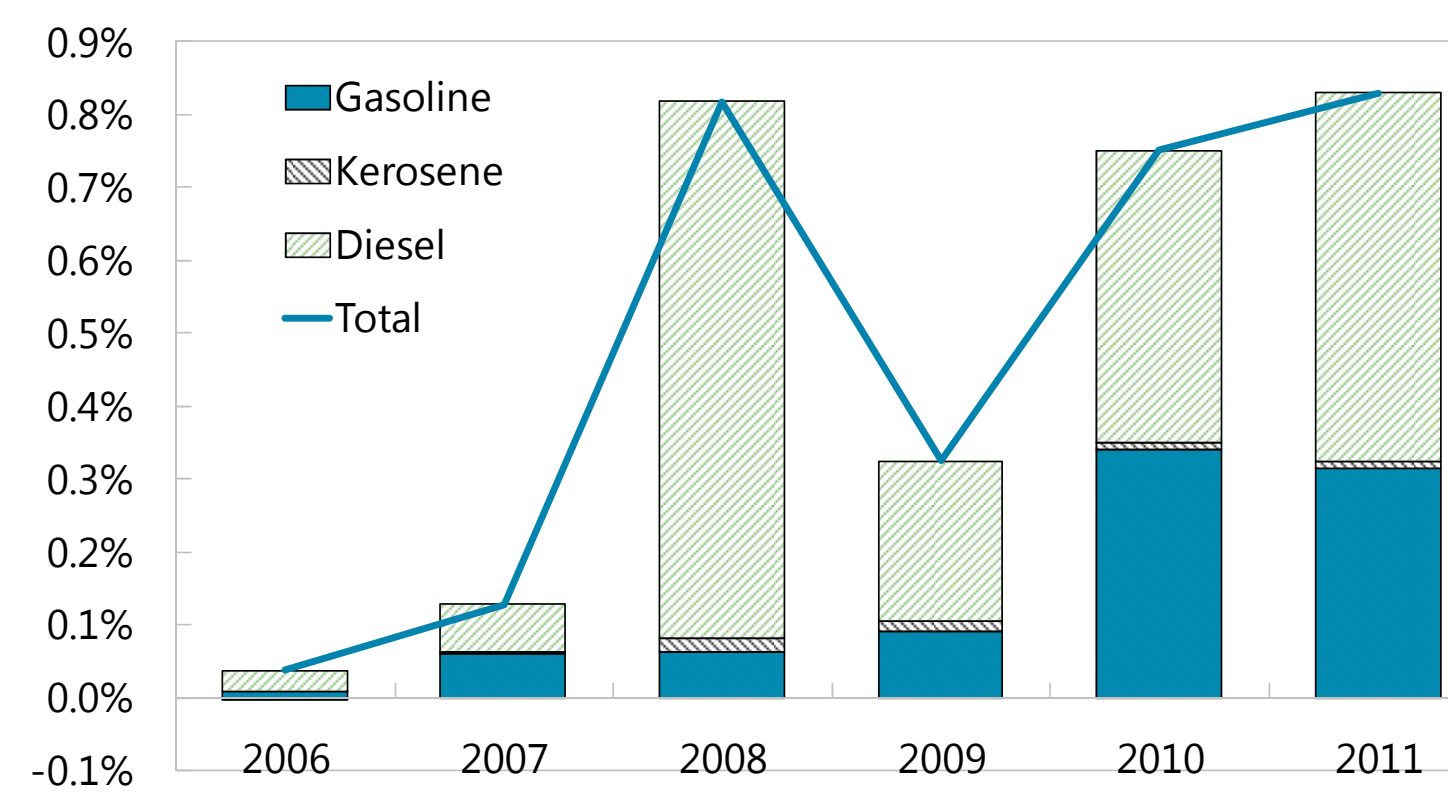

Sources: DRC Ministry of Economy; and IMF staff estimates.

Figure VII.2. DRC: Retail Prices Under Alternative Mechanisms, 2006-11

(Congolese francs per liter)

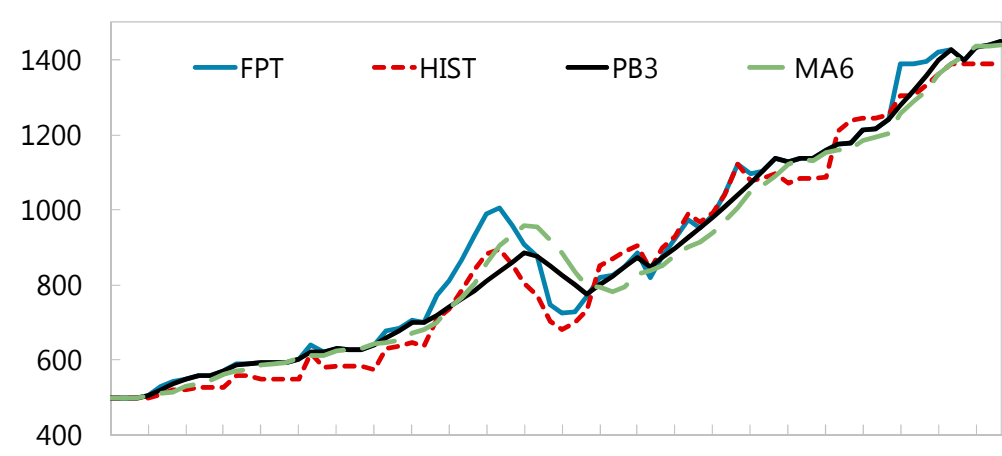

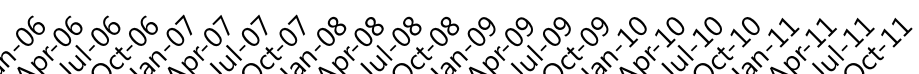

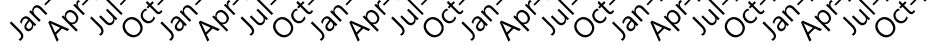

Source: IMF staff estimates.

Note: FPT: Full pass-through; HIST: Historical; PB3: 3 percent band; MA6:

6-month moving average. 
Figure VII.3. DRC: Net Taxes Under Alternative Mechanisms, 2006-11

(Congolese francs per liter)

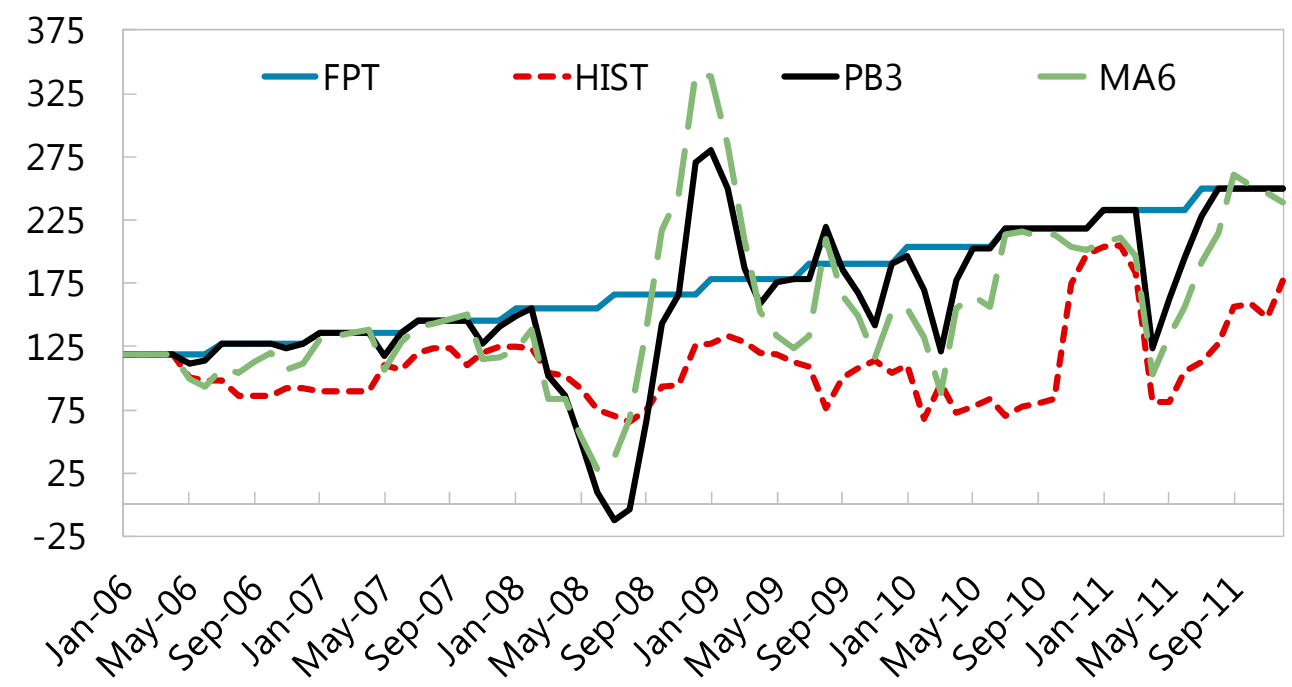

Source: IMF staff estimates.

Note: FPT: Full pass-through; HIST: Historical; PB3: 3 percent band; MA6:

6-month moving average.

\section{Table VII.1. DRC: Price and Tax Volatility Rankings of Alternative Mechanisms}

\begin{tabular}{|c|c|c|c|c|c|c|c|c|}
\hline Price volatility & FPT & HIST & MA2 & MA4 & MA6 & PB3 & PB5 & PB10 \\
\hline Std. deviation of monthly prices & 7 & 8 & 5 & 3 & 1 & 2 & 4 & 6 \\
\hline Avg monthly price change (sq) & 7 & 6 & 5 & 3 & 2 & 1 & 4 & 8 \\
\hline Largest single monthly price increase & 8 & 6 & 5 & 3 & 2 & 1 & 4 & 7 \\
\hline Avg of 2 largest monthly price inceases & 6 & 8 & 5 & 3 & 2 & 1 & 4 & 6 \\
\hline Avg of 3 largest monthly price increases & 7 & 8 & 5 & 3 & 2 & 1 & 4 & 6 \\
\hline Sum of price volatility rankings & 35 & 36 & 25 & 15 & 9 & 6 & 20 & 33 \\
\hline Rank order & 7 & 8 & 5 & 3 & 2 & 1 & 4 & 6 \\
\hline Tax volatility & FPT & HIST & MA2 & MA4 & MA6 & PB3 & PB5 & PB10 \\
\hline Std. deviation of taxes & 3 & 1 & 4 & 6 & 8 & 7 & 5 & 2 \\
\hline Avg monthly tax change (sq) & 1 & 3 & 4 & 6 & 8 & 7 & 5 & 2 \\
\hline Largest single monthly tax decrease & 1 & 7 & 3 & 4 & 6 & 8 & 5 & 2 \\
\hline Avg of 2 largest monthly tax decreases & 1 & 3 & 5 & 7 & 8 & 6 & 4 & 2 \\
\hline Avg of 3 largest monthly tax decreases & 1 & 3 & 5 & 6 & 8 & 7 & 4 & 2 \\
\hline Sum of tax volatility rankings & 7 & 17 & 21 & 29 & 38 & 35 & 23 & 10 \\
\hline Rank order & 1 & 3 & 4 & 6 & 8 & 7 & 5 & 2 \\
\hline Sum of all rankings & 42 & 53 & 46 & 44 & 47 & 41 & 43 & 43 \\
\hline Overall ranking & 2 & 8 & 6 & 5 & 7 & 1 & 3 & 3 \\
\hline
\end{tabular}




\begin{tabular}{|c|c|c|c|c|c|c|c|}
\hline \multicolumn{8}{|c|}{ Table VII.2. DRC: Fiscal Cost of Select Fuel Pricing Mechanisms } \\
\hline & 2006 & 2007 & 2008 & 2009 & 2010 & 2011 & 2006-2011 \\
\hline Historical & & & & & & & Total \\
\hline Fiscal cost per liter (CGF) & 22 & 32 & 65 & 71 & 112 & 97 & \\
\hline Fiscal cost (billion CGF) & 10 & 17 & 40 & 43 & 75 & 78 & 263 \\
\hline Fiscal cost ( $\%$ of GDP) & $0.2 \%$ & $0.3 \%$ & $0.6 \%$ & $0.5 \%$ & $0.6 \%$ & $0.5 \%$ & $2.8 \%$ \\
\hline \multicolumn{8}{|l|}{3 Percent Price Band } \\
\hline Fiscal cost per liter (CGF) & 1 & 3 & 62 & -8 & 13 & 20 & \\
\hline Fiscal Cost (billion CGF) & 1 & 2 & 38 & -5 & 9 & 16 & 60 \\
\hline Fiscal cost ( $\%$ of GDP) & $0.0 \%$ & $0.0 \%$ & $0.6 \%$ & $-0.1 \%$ & $0.1 \%$ & $0.1 \%$ & $0.8 \%$ \\
\hline Liters sold (million) & 460 & 537 & 610 & 602 & 667 & 807 & \\
\hline Nominal GDP (billion CGF) & 4,132 & 5,175 & 6,530 & 9,073 & 11,902 & 14,503 & \\
\hline
\end{tabular}




\section{References}

Arze del Granado, J., D. Coady, and R. Gillingham, 2010, "The Unequal Benefits of Fuel Subsidies: A Review of Evidence for Developing Countries," IMF Working Paper 10/202 (Washington: International Monetary Fund).

Coady, D., R. Gillingham, R. Ossowski, J. Piotrowski, S. Tareq, and J. Tyson, 2010, "Petroleum Product Subsidies: Costly, Inequitable, and Rising," IMF Staff Position Note, SPN/10/05 (Washington: International Monetary Fund).

Coady, D., A. Tuladhar, J. Arze del Granado, L. Eyraud, and L. Nemeth, 2011, "On the Design and Implementation of Automatic Fuel Pricing Mechanisms," Fiscal Affairs Department Technical Manual (Washington: International Monetary Fund).

Gupta, S., M. Verhoeven, R. Gillingham, C. Schiller, A. Mansoor, and J. Cordoba, 2000, Equity and Efficiency in the Reform of Price Subsidies: A Guide for Policymakers (Washington: International Monetary Fund). 


\section{Annex VIII. The Efficacy of Monetary Policy}

1. A number of factors contribute to the limited effectiveness of monetary policy in the DRC: the high level of dollarization, institutional and administrative weaknesses, and the lack of credibility.

- The monetary authorities' capacity to influence money demand through marketbased instruments is impaired because they affect mainly small balances in local currency in the formal banking system. Dollar deposits as share of total deposits exceed 90 percent-a very high level even compared with other dollarized economies — with a formal banking system that is itself small. Less than 1 percent of the population has access to a commercial bank (World Bank, 2010). Excluded from the banking system the population is by and large insensitive to changes in interest rates. The dominant sectors of the economy (export-oriented industries of which extractive industries account for 98 percent of total exports) and financial services are largely immune to exchange rate fluctuations, and the $\mathrm{BCC}^{\prime}$ 's policy rate does little to affect dollar-denominated credits to the economy. The formal localcurrency economy is relatively small and dominated by the public sector.

Consequently, the fiscal stance has a disproportionately large influence on monetary developments, and fiscal policy is outside the control of the central bank.

- The lack of financial and operational independence of the BCC leads to multiple and conflicting objectives. The BCC's reliance on government resources exposes it to political interference, and politicizes its decision-making, especially in periods of high inflation when interest rates on central bank bills and thus the cost of open market operations increase. The financial situation is further exacerbated by the slow progress in the BCC's structural reforms to reduce its structural deficit and to focus on its core mandate. Furthermore, poor and infrequent economic data inhibits monetary policy to accurately fine-tune monetary interventions and to be proactive to changes in inflation. Finally, shallow financial markets slow the monetary transmission mechanism and delay the impact on inflation. ${ }^{1}$

\section{Among options to improve the effectiveness of monetary policy, improvements to the current monetary} policy regime are preferable to changing the monetary policy regime altogether. The institutional reforms required to improve the effectiveness of the current monetary policy framework are significant, and it will take years if not a decade or more before dollarization declines and financial markets develop to such an extent that monetary policy becomes highly effective and independent. Nonetheless, staff argues that this is still the most viable option for the DRC for several reasons:

- Seeking to borrow the credibility of a foreign central bank through a fixed peg in the context of weak institutions and a short track record of macroeconomic discipline makes the currency vulnerable to

\footnotetext{
${ }^{1}$ Broad money in the DRC at 17 percent of GDP is low by international standards and compared to other SSA countries (48 percent of GDP).
} 
speculative attacks. Abandoning a peg would further harm the weak credibility of the central bank.

- $\quad$ By opting for a stronger fixed peg-a currency board or full dollarization-the DRC would require US\$2-4 billion, or the equivalent of the domestic currency and whatever level of international reserves the authorities consider necessary to fulfill its lender of last resort function. Under the current circumstances of a tight budget and closed international financial markets, the DRC does not seem to be able to raise such large amounts of capital.

- The DRC's exports are concentrated in a few commodities with historically volatile prices. Under the current monetary policy regime the DRC keeps the option of a real devaluation to partially absorb external shocks and thus limit the adverse impact on growth and employment. This option would be lost with any type of fixed exchange rate regime.

- A fixed exchange rate can conflict with a low inflation target if the real exchange rate appreciates as a result of a rapid productivity growth in the tradable sector.

- Finally, the DRC is undergoing profound structural changes. Its economy will eventually diversify and its export markets will develop. A fixed exchange rate would lock the country into a currency which may not be optimal at future stages of development.

\section{To improve the effectiveness of} monetary policy in the long run, a number of reforms will be needed to strengthen the independence of the central bank and its focus on its core mandate, improve the central bank's data availability and research capacity, deepen financial markets, as well as to encourage de-dollarization. These improvements would enable a more flexible and modernized monetary policy framework, anchored by a coherent forward-looking view where monetary aggregates are monitored and analyzed systematically along with a broad array of other macroeconomic and financial indicators, including inflation, output, interest rates, and the exchange rate. As most of these reforms will take time, the current constraints to an effective and independent monetary policy are likely to remain for some time.

\section{Measures that encourage rather} than force de-dollarization are likely to be the most effective. Even with continued macroeconomic stability international experience shows that dollarization will take years or decades to reverse. The preferable market based de-dollarization measures (socalled pull factors) could include strengthening liquidity management and instruments through the introduction of medium term paper in local currency; and shifting most government transactions into local currency; issuing bank notes with higher denominations to reduce transactions costs and ease the physical use of local currency for larger transactions. Forced de-dollarization measures (push factors), such as mandatory use of local currency or regulations against the use of foreign currency should be avoided because they may result in capital outflows rather than de-dollarization. Along the same lines, reserve requirements that match the currency composition of deposits may be needed for prudential reasons, even though they further reduce the use of the local currency. 


\section{INTERNATIONAL MONETARY FUND}

\section{DEMOCRATIC REPUBLIC OF THE CONGO}

\section{STAFF REPORT FOR THE 2012 ARTICLE IV}

CONSULTATION-INFORMATIONAL ANNEX

Prepared By

\section{CONTENTS}

I. RELATIONS WITH THE IMF 2

II. JMAP IMPLEMENTATION MATRIX $\underline{8}$

III. STATISTICAL ISSUES $\underline{9}$ 


\section{RELATIONS WITH THE IMF}

As of July 31, 2012

Membership Status: Joined September 28, 1963; Article VIII

General Resources Account:

Quota

Fund holdings of currency

SDR Department:

Net cumulative allocation

Holdings

Outstanding Purchases and Loans:

ESF RAC loan

ECF Arrangement

Latest Financial Arrangements:

Type

$$
\begin{gathered}
\text { Approval } \\
\text { Date }
\end{gathered}
$$

$\mathrm{ECF}^{1}$

$\mathrm{ECF}^{1}$

Dec 11, 2009

June 12, 2002

Stand-By

June 09, 1989

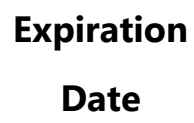

Dec 10, 2012

Mar 31, 2006

June 08, 1990
SDR Million

533.00

533.00

SDR Million

510.86

352.38

SDR Million

108.16

200.64
Percent of

\section{Quota}

100.00

100.00

Percent of

Quota

100.0

69.98

Percent of

Quota

20.29

37.64

\section{Amount \\ Approved}

(SDR Million)

346.45

580.00

116.40
Amount Drawn

(SDR Million)

197.97

553.47

75.00

\footnotetext{
${ }^{1}$ Formerly PRGF.
} 


\section{Projected Payments to Fund: ${ }^{2}$}

(SDR million; based on existing use of resources and present holdings of SDRs):

\section{Forthcoming}

$\begin{array}{lrrrrr} & \mathbf{2 0 1 2} & \mathbf{2 0 1 3} & \mathbf{2 0 1 4} & \mathbf{2 0 1 5} & \mathbf{2 0 1 6} \\ \text { Principal } & 0.00 & 0.00 & 0.00 & 27.46 & 56.35 \\ \text { Charges/interest } & 0.08 & 0.42 & 0.92 & 0.90 & 0.79 \\ \text { Total } & 0.08 & 0.42 & 0.92 & 28.36 & 57.13\end{array}$

\section{Implementation of HIPC Initiative:}

I. Commitment of HIPC assistance

Enhanced Framework

Decision point date July 2003

Assistance committed by all creditors (US\$ millions) $)^{3}$

Of which: IMF assistance (US\$ millions)

(SDR equivalent millions)

Completion point date

July 2010

II. Disbursement of IMF assistance (SDR millions)

Assistance disbursed to the member

Interim assistance

Completion point balance

Additional disbursement of interest income ${ }^{4}$

Total disbursements

\footnotetext{
${ }^{2}$ When a member has overdue financial obligations outstanding for more than three months, the amount of such arrears will be shown in this section.

${ }^{3}$ Assistance committed under the original framework is expressed in net present value (NPV) terms at the completion point, and assistance committed under the enhanced framework is expressed in NPV terms at the decision point. Hence these two numbers cannot be added.

${ }^{4}$ Under the enhanced framework, an additional disbursement is made at the completion point corresponding to interest income earned on the amount committed at the decision point but not disbursed during the interim period.
} 


\section{Implementation of MDRI Assistance:}

I. $\quad$ MDRI-eligible debt (SDR Million) ${ }^{5}$

Financed by: MDRI Trust $\quad 0.00$

Remaining HIPC resources $\quad 248.08$

II. Debt Relief by Facility (SDR Million)

Eligible Debt

$\begin{array}{lllr}\text { Delivery Date } & \text { GRA } & \text { PRGT } & \text { Total } \\ \text { July } 2010 & \text { N/A } & 248.08 & 248.08\end{array}$

\section{Exchange Rate Arrangement:}

The currency of the Democratic Republic of the Congo (DRC) is the Congo franc. The de jure exchange rate arrangement is floating, although the IMF classifies the Congo franc as an "other managed arrangement". At end-June 2012, the rate was US $\$ 1=$ CF 919.32 .

Effective February 10, 2003, the DRC accepted the obligations of Article VIII, Sections 2 (a), 3, and 4, of the Fund's Articles of Agreement; however, the DRC maintains measures that give rise to one exchange rate restriction and one multiple currency practice subject to Fund approval. The exchange restriction involves an outstanding net debt position against other contracting members under the inoperative regional payments agreement with the Economic Community of the Great Lakes Countries. The multiple currency practice relates to a fixed exchange rate set quarterly applying to transactions through a bilateral payments agreement with Zimbabwe.

\section{Last Article IV Consultation:}

Consultations with the DRC are held in accordance with the provisions of the 2010 Decision on consultation cycles approved in September 2010. The last Article IV consultation was concluded by the Executive Board on December 11, 2009.

\section{Safeguards Assessment:}

An update safeguards assessment of the Banque Centrale du Congo (BCC) was completed in April 2010, which found that while most of the 2008 recommendations had been implemented, significant risks remained. The BCC continued to lack autonomy from the government and was in need of recapitalization, and the absence

\footnotetext{
${ }^{5}$ The MDRI provides 100 percent debt relief to eligible countries that qualified for the assistance. Grant assistance from MDRI Trust and HIPC resources provide debt relief to cover the full stock of debt owed to the Fund as of end-2004 that remains outstanding at the time the member qualifies for such debt relief.
} 
of an independently defined financing reporting framework continued to impair transparency. The BCC agreed broadly with the priority recommendations of the assessment, and has since initiated important reforms. In March 2011, the Ministry of Finance completed the first phase of the BCC's recapitalization, bringing its net worth to zero. Additionally, the BCC believes that they are still on target to issue their 2012 financial statements in accordance with IFRS, although the transition process has been protracted.

Technical Assistance:

\section{STA}

FY 2007 - Real sector statistics

FY 2008 - Real sector statistics

FY 2009 - National accounts

FY 2010 - Monetary statistics and national accounts

FY 2011 - Monetary statistics and national accounts

FY 2012 - Monetary statistics, national accounts and balance of payments.

\section{MCM}

Capacity building assistance to the central bank

FY 2012 - One mission (TA needs assessment)

Strengthening capacity in Anti-Money Laundering/Combating Financial Terrorism (AML/CFT)

FY 2005 - One short visit

FY 2006 - Two short visits

Strengthening capacity in monetary policy implementation, exchange operations, and liquidity management

FY 2005 - Three short visits

FY 2006 - Two short visits

FY 2007 - Seven short visits

FY 2008 - Three short visits

FY 2009 - One short visit

FY 2011 - Three short visits

FY 2012 - Two short visits (of which one on currency reform and handling)

Restructuring and reorganization of the central bank

FY 2009 - One short visit

FY 2010 - One short visit

FY 2011 - Four short visits

FY 2012 - One mission.

Strengthening capacity in banking supervision and financial sector development

FY 2005 - One short visit on bank supervision and regulation

FY 2006 - One short visit on internal audit

FY 2007 - Action plan for developing the financial system 
FY 2008 - One short visit on TA assessment

FY 2008 - Two short visits on bank supervision and regulation

FY 2008 - One short visit on internal audit

FY 2010 - Three short visits on bank supervision and regulation

FY 2011 - Three short visits

FY 2011 - Two short visits on bank supervision and regulation

FY 2012 - One mission.

LEG

FY 2012 - Two missions.

FAD

Public financial management

FY 2007 - Short visit

FY 2009 - Short visit

FY 2010 - Short visit

FY 2011 - Short visit

FY 2012 - One short visit (MNRW TTF diagnostic)

Revenue administration

FY 2005 - One short visit

FY 2006 - One short visit

FY 2007 - One short visit

FY 2008 - One short visit

FY 2009 - One short visit

FY 2011 - One short visit (VAT diagnostics)

FY2012 - One short visit (MNRW TTF diagnostic)

Fiscal decentralization

FY 2005 - Short visit

Tax Policy

FY 2012 - One short visit (MNRW TTF diagnostic)

\section{AFRITAC Central}

Public financial management

FY 2009 - Four missions

FY 2010 - Three missions

FY 2011 - One mission

FY 2012 - Five missions and one short visit

Tax administration

FY 2008 - One mission

FY 2009 - Two missions

FY 2010 - Three missions

FY 2011 - Two missions

CInternational Monetary Fund. Not for Redistribution 
FY 2012 - One mission

Bank supervision

FY 2008 - Three missions

FY 2009 - Three missions

FY 2010 - Three missions

FY 2011 - Two missions and one short visit

FY 2012 - Five missions

Public debt

FY 2008 - One mission and one short visit

FY 2009 - One short visit

FY 2010 - Three missions

Statistics

FY 2009 - One short visit

FY 2010 - Two missions

FY 2011 - One mission and one short visit

National Accounts

FY 2009 - One short visit

FY 2010- Two missions

FY 2011 -One mission and one short visit

FY 2012 - One mission

\section{Long-term Resident Experts:}

Expenditure management

Mr. Catalan

Jan. 2002 - June 2004

Mr. Bremeersch

Jan. 2002 - Jan. 2004

Mr. Nguenang

Sept. 2004 - July 2008

Tax administration

Mr. Fossat

Sept. 2005 - Jan. 2007

VAT preparation and

Mr. Ramarozatovo

Feb. 2011 - ongoing

implementation

Advisor to Governor of BCC

Mr. Nyssens

Jan. 2004 - Jan. 2005

Mr. d'Ambrières

Feb. 2006 - June 2006

Mr. Traoré

June 2010 - ongoing

Capacity building of BCC

Mr. Pauwels

Sept. 2004 - Mar. 2007

Statistics

Mr. Métreau

Oct. 2004 - Oct. 2005

Mr. Gbossa

Dec. 2005 - 2008

Resident Representative: Mr. Jahjah assumed his duties in January 2009. 


\section{JMAP IMPLEMENTATION MATRIX}

As of July 10, 2012

1. The IMF and World Bank teams discussed a joint managerial action plan under the JMAP initiative.

2. The World Bank's FY13 work program entails additional financing for projects in the transport, health and governance sector as well as two new projects; (i) a civil service rejuvenation project and (ii) a growth poles project.

3. The IMF's work program entails a three-year arrangement under the Extended Credit Facility (ECF) approved by the IMF's Executive Board in December 2009 and an Art. IV consultation.

4. Both institutions are supporting an FSAP that could take place during FY14.

\begin{tabular}{|c|c|c|c|c|c|c|c|}
\hline & \multirow[t]{2}{*}{ Title } & \multicolumn{2}{|r|}{ Products } & \multicolumn{2}{|c|}{$\begin{array}{l}\text { Provisional timing of } \\
\text { missions }\end{array}$} & \multicolumn{2}{|c|}{$\begin{array}{l}\text { Actual or expected } \\
\text { delivery date }\end{array}$} \\
\hline \multicolumn{7}{|c|}{ A. Mutual information on relevant work program } & \\
\hline \multirow[t]{6}{*}{ - } & \multirow{6}{*}{$\begin{array}{l}\text { World Bank } \\
\text { work program } \\
\text { in the next } \\
12 \text { months }\end{array}$} & $\bullet$ & $\begin{array}{l}\text { Civil Service Rejuvenation project } \\
\text { Investment Lending Operation }\end{array}$ & $\bullet$ & October 2012 & $\bullet$ & FY13 \\
\hline & & $\bullet$ & $\begin{array}{l}\text { Additional Financing for Governance } \\
\text { Enhancement Project }\end{array}$ & $\bullet$ & October 2012 & $\bullet$ & FY13 \\
\hline & & $\bullet$ & $\begin{array}{l}\text { Additional financing for the Multimodel } \\
\text { transport project }\end{array}$ & $\bullet$ & October 2012 & $\bullet$ & FY13 \\
\hline & & $\bullet$ & $\begin{array}{l}\text { Additional financing for the Health } \\
\text { Project (PARSS) }\end{array}$ & $\bullet$ & October 2012 & $\bullet$ & FY13 \\
\hline & & $\bullet$ & Urban project & $\bullet$ & October 2012 & $\bullet$ & FY13 \\
\hline & & $\bullet$ & Statistics project & $\bullet$ & December 2012 & $\bullet$ & FY13 \\
\hline \multirow[t]{2}{*}{$\bullet$} & \multirow{2}{*}{$\begin{array}{l}\text { IMF work } \\
\text { program in the } \\
\text { next } \\
12 \text { months }\end{array}$} & $\bullet$ & $\begin{array}{l}\text { Fourth and fifth review of the ECF and } \\
\text { Article IV consultation }\end{array}$ & $\bullet$ & June 2012 & $\bullet$ & September 2012 \\
\hline & & $\bullet$ & Sixth review of the ECF & $\bullet$ & November 2012 & $\bullet$ & January 2013 \\
\hline
\end{tabular}

\section{B. Requests for work program inputs}

\begin{tabular}{|c|c|c|c|c|c|c|c|}
\hline$\bullet$ & $\begin{array}{l}\text { Fund request } \\
\text { to Bank }\end{array}$ & $\bullet$ & $\begin{array}{l}\text { Provide comments on implications of } \\
\text { sector-specific issues, including } \\
\text { developments in the mining, energy, } \\
\text { telecom and agricultural sectors and the } \\
\text { reform of public enterprises. }\end{array}$ & $\bullet$ & None & $\bullet$ & FY 13 \\
\hline$\bullet$ & $\begin{array}{l}\text { Bank request } \\
\text { to Fund }\end{array}$ & $\bullet$ & $\begin{array}{l}\text { Macroeconomic scenarios for investment } \\
\text { operations }\end{array}$ & $\bullet$ & $\begin{array}{l}\text { Managed by Kinshasa } \\
\text { office }\end{array}$ & $\bullet$ & FY 13 \\
\hline
\end{tabular}

\section{Agreement on joint products and missions}

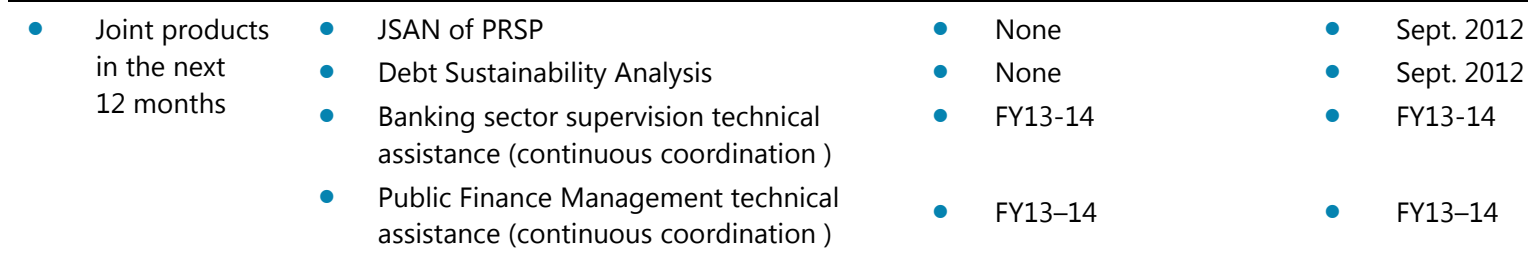




\section{STATISTICAL ISSUES}

\section{Assessment of Data Adequacy for Surveillance}

General: Data provision to the Fund is broadly adequate for surveillance and program monitoring purposes, but upgrades to the national accounts, balance of payments, and government finance statistics would be helpful. In spite of difficulties, the authorities have continued to produce an array of economic and financial statistics, most of which are published in the annual report of the Central Bank of the Congo (BCC) or its monthly statistical bulletin.

National accounts: Annual national accounts are compiled in constant and current prices by the Directorate of Research of the BCC and published yearly. The compilation methodology conforms to the System of National Accounts 1968 (1968 SNA) and is based on the balance sheets of enterprises and surveys of public and semipublic enterprises and agencies, most of which date from the late 1980s. The activities of the traditional sector (including the informal sector) are estimated with extrapolation techniques using industry-specific data that are outdated. As a result, GDP is likely to be underestimated.

With assistance from AFRITAC, work on the national accounts is underway to update the base year to 2005 , incorporate the results of the 2004/5 1-2-3 household survey, as well as implement the methodological recommendations of the 1993 SNA. While outputs of this work remain to be validated and officially published, as expected, the 2005 GDP estimates show a considerable upward revision compared to previously published estimates.

Price statistics: Consumer price indices (CPI) are calculated for Kinshasa by the BCC, the National Statistics Institute (INS), the Institute of Economic and Social Research (IESR), and the Economics Section of the U.S. Embassy in Kinshasa. In December 2010, the BCC adopted a new harmonized-CPI index for Kinshasa based on the 2004/05 1-2-3 household survey. In 2012, the INS began publishing a national CPI index, based upon the 2004/05 1-2-3 household survey and prices prevailing in provincial capitals.

Government finance statistics: The BCC produces aggregated monthly statistics on a cash basis based on its own accounting for the government cash operations. The treasury produces two sets of monthly statistics based on its own records: one relates to the transactions executed through the BCC, the other set attempts to consolidate operations through commercial bank accounts and off-budget operations. These statistics do not rely on an integrated double-entry public accounting system and do not provide sufficient details about the nature of expenditures owing to problems in the expenditure chain. However, the treasury has started to produce quarterly expenditure data reports by ministry and institution.

The current work on tax administration and expenditure control is expected to improve the quality and timeliness of fiscal statistics. In parallel with technical assistance on public expenditure management, a STA multisector statistics advisor has been advising on government finance statistics, producing improvements in the quality of the statistics. Furthermore, adoption of a simplified doubleentry bookkeeping system should significantly improve the availability of data on government financial operations.

In 2003 the DRC reported annual data up to 2002 for the IMF's Government Finance Statistics Yearbook in the format of the Government Finance Statistics Manual 2001 but has not reported subsequently. However, work is underway to resume submission of data to the GFSY, beginning with data for 2010. 
Monetary statistics: The BCC Directorate of Statistics regularly produces timely monetary statistics. These statistics are now generally reliable but some minor problems remain with the classification of accounts.

Since STA money and banking statistics mission of June 2000, BCC reporting of data for publication in the IMF's International Financial Statistics (IFS) has improved and monetary data are currently reported on time. A 2003 multisector statistics mission recommended a new chart of accounts for the BCC and other deposit-taking institutions, including the information needed for improved sectorization of economic units and classification of financial assets. The new chart of accounts for the central bank was applied beginning in 2007 and the new chart of accounts for commercial banks was applied in January 2011. The August 2009 MFS mission developed the new standardized report form (SRF) 1SR on central bank data. The December 2010 MFS mission developed the SRF 2SR on commercial bank data based on the past chart of accounts up to December 2010. The August 2011 mission developed and almost finalized SRF 2SR on commercial banks data based on the new chart of accounts. At this stage, source data from commercial banks are not fully reliable as respondents do not completely master the new sectoral chart of accounts yet. Also, some inconsistencies in reciprocal claims and liabilities between SRF 1SR and SRF 2SR must be reduced. An MFS mission, scheduled in FY2013, should occur by end-2012 and will help finalize the official migration to the SRFs format for data on central bank and data on other depository corporations, commercial banks at least.

Balance of payments: Annual balance of payments statistics are compiled with information on the exports and imports of large public and semipublic enterprises, BCC payment records, and a survey of the foreign operations of residents. The estimates also take account of information on the informal sector and foreign aid flows provided by the World Bank and the local UN Development Program office, which collects the data from the European Union, embassies, and nongovernmental organizations. In effect balance of payments statistics are compiled using data from different sources, supplemented by estimates. However, in September 2010, the authorities took decision to create an interministerial committee, lead by the central bank and also including representatives from customs, and the National Statistics Institute and key sectors, to improve the collection and the validation of balance of payment data and ensure its regular publication. No external sector data are reported to STA for publication.

\section{Data Standards and Quality}

Since April 24, 2004, the DRC participates in the General Data Dissemination System. The metadata needs to be updated. 


\begin{tabular}{|c|c|c|c|c|c|}
\hline \multicolumn{6}{|c|}{$\begin{array}{l}\text { The Democratic Republic of the Congo: Table of Common Indicators Required for Surveillance } \\
\text { (As of July 10,2012) }\end{array}$} \\
\hline & $\begin{array}{c}\text { Date of } \\
\text { latest } \\
\text { observation }\end{array}$ & $\begin{array}{l}\text { Date } \\
\text { received }\end{array}$ & $\begin{array}{c}\text { Frequency } \\
\text { of Data }^{6}\end{array}$ & $\begin{array}{c}\text { Frequency of } \\
\text { Reporting }\end{array}$ & $\begin{array}{l}\text { Frequency of } \\
\text { Publication }{ }^{6}\end{array}$ \\
\hline Exchange Rates & 10-Jul-12 & 10-Jul-12 & $\mathrm{D}$ & $\mathrm{D}$ & $\mathrm{D}$ \\
\hline International Reserve Assets and Reserves & Apr-12 & Jun-12 & M & M & M \\
\hline Liabilities of the Monetary Authorities ${ }^{1}$ & Apr-12 & Jun-12 & M & M & M \\
\hline Reserve/Base money & Apr-12 & Jun-12 & M & M & M \\
\hline Broad money & Apr-12 & Jun-12 & M & M & M \\
\hline Central Bank Balance Sheet & Apr-12 & Jun-12 & M & M & M \\
\hline Consolidated Balance Sheet of the Banking System & Apr-12 & Jun-12 & M & M & M \\
\hline Interest Rates ${ }^{2}$ & Apr-12 & Jun-12 & M & 1 & I \\
\hline Consumer Price Index & 25-Jun-12 & 9-Jul-11 & W & W & W \\
\hline Central Government revenue, expenditure and financing ${ }^{3}$ & Apr-11 & May-11 & M & M & M \\
\hline $\begin{array}{l}\text { Stock of Central Government and Central Government- } \\
\text { Guaranteed Debt }{ }^{4}\end{array}$ & 2011 & Jun-12 & A & A & 1 \\
\hline External Current Account Balance & 12-Mar & Jun-12 & A & A & 1 \\
\hline Exports and Imports of Goods and Services & Mar-12 & Jun-12 & A & $A$ & 1 \\
\hline GDP/GNP & 2011 & Jun-12 & A & A & 1 \\
\hline Gross External Debt & 2011 & Jun-12 & A & A & 1 \\
\hline International Investment Position ${ }^{5}$ & $\mathrm{~N} / \mathrm{A}$ & $\mathrm{N} / \mathrm{A}$ & $\mathrm{N} / \mathrm{A}$ & $\mathrm{N} / \mathrm{A}$ & $N / A$ \\
\hline
\end{tabular}

${ }^{1}$ Any reserves assets that are pledged or otherwise encumbered should be specified separately. Also, data should comprise short-term liabilities linked to a foreign currency but settled by other means as well as the national values of financial derivatives to pay and receive foreign currency, including those linked to a foreign curreny but settled by other means.

${ }^{2}$ Both market-based and officially-determined, including discount rates, money markets rates, rates on treasury bills, notes and bonds.

${ }^{3}$ Foreign, domestic bank, and domestic nonbank financing.

${ }^{4}$ Including currency and maturity composition. In light of recent misreporting on external arrears, the authorities have agreed to henceforth report this data on a monthly basis.

${ }^{5}$ Includes external gross financial asset and libility positions vis-à-vis nonresidents. Due to lack of capacity, this data is not provided by the DRC.

${ }^{6}$ Daily(D), Weekly (W), Monthly (M), Quarterly (Q), Irregular (I), Not available (NA). 


\section{INTERNATIONAL MONETARY FUND}

Public Information Notice

EXTERNAL

RELATIONS

DEPARTMENT

Public Information Notice (PIN) No. 12/115

FOR IMMEDIATE RELEASE

September 27, 2012

International Monetary Fund

$70019^{\text {th }}$ Street, NW

Washington, D. C. 20431 USA

\section{IMF Executive Board Concludes 2012 Article IV Consultation with the Democratic Republic of the Congo}

On September 24, 2012, the Executive Board of the International Monetary Fund (IMF) concluded the 2012 Article IV consultation with the Democratic Republic of the Congo (DRC). ${ }^{1}$

\section{Background}

In the past few years, economic performance in the DRC has shown marked improvement although progress in alleviating poverty and meeting the Millennium Development Goals has lagged. Prudent macroeconomic policies and structural reforms have underpinned this performance and led to the DRC receiving debt relief under the enhanced Heavily Indebted Poor Countries (HIPC) Initiative in mid-2010.

The DRC's weak financial linkages with the euro area countries have largely shielded it from the turmoil there, allowing real gross domestic product (GDP) growth to expand by about 7 percent in 2010-11. Strong trade and investment inflows from non-European countries, driven mainly by the mining sector, have provided the main impetus to growth. Inflation was well above the single-digit target of the Central Bank of the Congo (BCC) in 2011 largely because of external factors, but has recently slowed to about 10 percent through July 2012.

\footnotetext{
${ }^{1}$ Under Article IV of the IMF's Articles of Agreement, the IMF holds bilateral discussions with members, usually every year. A staff team visits the country, collects economic and financial information, and discusses with officials the country's economic developments and policies. On return to headquarters, the staff prepares a report, which forms the basis for discussion by the Executive Board. At the conclusion of the discussion, the Managing Director, as Chairman of the Board, summarizes the views of Executive Directors, and this summary is transmitted to the country's authorities. An explanation of any qualifiers used in summings up can be found here: http://www.imf.org/external/np/sec/misc/qualifiers.htm.
} 
The external sector improved significantly as a result of HIPC debt relief but the DRC remains at high risk of debt distress because of its vulnerability to the volatile terms of trade, high import dependency on food and capital goods, and a narrow export base. Weak debt management also increases the risk of again accumulating an unsustainable level of debt. The current account deficit widened in 2011 to about $11 \frac{1}{2}$ percent of GDP as commodity prices weakened from their high levels in 2010 and the value of imports increased, especially for food, fuel, and capital goods.

Macroeconomic policies have provided strong support to recent performance, with the DRC having rebuilt many of its buffers. In particular, fiscal dominance has been reduced over the past few years, owing to the government's expenditure discipline and commitment to zero (net) financing of the budget from the BCC. This has helped break a vicious inflation-exchange rate depreciation cycle and brought the domestic fiscal deficit down from 21/2 percent of GDP in 2009 to a projected deficit of 0.9 percent of GDP in 2012. Strong expenditure discipline has been an essential element in the authorities' efforts to achieve consolidation, which was necessary in the context of the limited external financial support to the DRC—reflecting donors concerns over governance, especially in the natural resource sector-and slow progress in raising more domestic revenue. Recently, however, weaknesses in the budget process, inflated revenue projections, and a corresponding large expenditure envelope pose a risk to fiscal credibility and point to the need to bolster and better entrench public financial management reforms.

The improvement in the fiscal situation has also helped the BCC control liquidity and fight inflation. The BCC aims at containing inflation through the issuance of central bank bills but data and capacity problems as well as political pressures have made it hesitant at times to proactively tighten monetary policy.

Although progress in structural reform has been mixed, some reforms in key areas are supportive of better economic performance. These include efforts to improve public financial management, strengthen governance and transparency in the natural resource sector, and enhance the BCC's independence and financial stability and development of the financial sector more generally. Still, other reforms have lagged and new issues have emerged, including weaknesses in external debt management (emergence of external payments arrears) and accountability and transparency in the operations of state-owned enterprises (SOEs) in extractive industries. These weaknesses could undermine recent progress and have contributed to the delay in completing the fourth and fifth reviews under the authorities' Extended Credit Facility arrangement.

\section{Executive Board Assessment}

Executive Directors welcomed the authorities' improved macroeconomic management, which contributed to higher growth, lower inflation, and a stronger external position. However, poverty remains widespread, the economy is vulnerable to domestic and external risks, and program 
delays are cause for concern. Directors called for steadfast efforts to preserve fiscal credibility, strengthen the monetary policy framework, and improve the governance of extractive industries.

Directors commended the authorities' progress in consolidating the fiscal position, supported by the commitment to forgo financing of the fiscal deficit from the central bank. They encouraged the authorities to maintain this fiscal anchor but expressed concern that the 2012 budget threatened hard-won credibility gains. Accordingly, they called for monitoring budget execution rigorously, reducing discretionary spending if revenue projections fail to materialize, and making further efforts to increase domestic revenues, particularly from extractive industries. Directors also encouraged the authorities to strengthen debt management and consider external borrowing only at highly concessional terms.

Directors encouraged the central bank to manage liquidity tightly to further dampen inflation expectations and guard against second-round effects from the recent increase in global food and fuel prices. They also agreed that completion of recapitalization, divestment of non-core activities, and greater operational transparency would strengthen central bank independence and improve the effectiveness of monetary policy.

Directors considered that the current monetary framework and floating exchange rate regime remain appropriate. However, given the high level of dollarization and the central bank's limited ability to act as lender of last resort in the event of a deposit run, a further build up of international reserves is warranted. While the external sector has improved recently, the country remains vulnerable to external shocks due to its narrow export base and lack of access to international capital markets.

Directors noted that the financial sector is underdeveloped, limiting financial intermediation and the transmission mechanism of monetary policy. They advised the authorities to improve bank supervision, enforcement of prudential requirements, data quality, and the central bank's analytical capacity. Directors welcomed the authorities' request to participate in the Financial Sector Assessment Program.

Directors emphasized that efforts to improve governance and transparency in extractive industries, and the business climate more generally, must be stepped up if the country is to maximize the benefits of its vast natural resource wealth. In particular, the authorities must move quickly to address gaps recently exposed by the commercialization of state-owned enterprises in the natural resource sector.

Public Information Notices (PINs) form part of the IMF's efforts to promote transparency of the IMF's views and analysis of economic developments and policies. With the consent of the country (or countries) concerned, PINs are issued after Executive Board discussions of Article IV consultations with member countries, of its surveillance of developments at the regional level, of post-program monitoring, and of ex post assessments of member countries with longer-term program engagements. PINs are also issued after Executive Board discussions of general policy matters, unless otherwise decided by the Executive Board in a particular case. 
Democratic Republic of the Congo: Selected Economic Indicators, 2009-13

\begin{tabular}{|c|c|c|c|c|c|}
\hline & 2009 & 2010 & 2011 & $\begin{array}{r}2012 \\
\text { Est. }\end{array}$ & $\begin{array}{l}2013 \\
\text { Proj. }\end{array}$ \\
\hline GDP and prices & \multicolumn{5}{|c|}{ (Annual percentage change) } \\
\hline Real GDP & 2.8 & 7.2 & 6.9 & 7.2 & 8.2 \\
\hline GDP deflator & 35.1 & 22.1 & 13.4 & 6.6 & 6.7 \\
\hline Consumer prices, period average & 46.2 & 23.5 & 15.5 & 10.9 & 9.5 \\
\hline Consumer prices, end-of-period & 53.4 & 9.8 & 15.4 & 9.9 & 9.0 \\
\hline \multicolumn{6}{|l|}{ External Sector } \\
\hline Export, f.o.b. (U.S. dollars) & -33.6 & 90.4 & 18.1 & -5.6 & 17.2 \\
\hline Imports, f.o.b. (U.S. dollars) & -26.3 & 54.9 & 21.5 & -7.8 & 9.2 \\
\hline Export volume & -1.3 & 47.4 & 21.0 & 8.7 & 19.5 \\
\hline Import volume & -12.9 & 41.7 & 5.4 & -6.2 & 1.6 \\
\hline Terms of trade & & 21.7 & -14.1 & -11.6 & 0.2 \\
\hline Money and credit & \multicolumn{5}{|c|}{$\begin{array}{l}\text { (Annual change in percent of beginning-of-period } \\
\text { broad money; unless indicated) }\end{array}$} \\
\hline Broad money & 50.4 & 30.8 & 23.1 & 14.6 & 17.0 \\
\hline Net foreign assets & 17.0 & 63.2 & -3.7 & 10.2 & 8.4 \\
\hline Net domestic assets & 33.3 & -32.1 & 25.8 & 4.6 & 8.7 \\
\hline Domestic credit & 13.5 & -28.9 & 19.1 & 10.3 & 9.9 \\
\hline Central government finance & \multicolumn{5}{|c|}{ (Percent of GDP; unless otherwise indicated) } \\
\hline Total government revenue & 16.8 & 19.0 & 18.9 & 22.3 & 21.7 \\
\hline Grants & 7.5 & 14.1 & 8.5 & 8.6 & 6.8 \\
\hline Total government expenditure & 26.0 & 27.3 & 29.1 & 33.5 & 31.8 \\
\hline Domestic fiscal balance (cash basis) & -2.5 & 0.9 & -1.7 & -0.9 & -0.4 \\
\hline $\begin{array}{l}\text { Overall fiscal balance (payment order } \\
\text { basis, incl. grants) }\end{array}$ & -1.7 & 5.8 & -1.8 & -2.6 & -3.4 \\
\hline $\begin{array}{l}\text { Overall fiscal balance (cash basis, incl. } \\
\text { grants) }\end{array}$ & -2.7 & 4.5 & -2.2 & -3.0 & -3.9 \\
\hline
\end{tabular}


Democratic Republic of the Congo: Selected Economic Indicators, 2009-13

\begin{tabular}{|c|c|c|c|c|c|}
\hline & 2009 & 2010 & 2011 & $\begin{array}{r}2012 \\
\text { Est. }\end{array}$ & $\begin{array}{l}2013 \\
\text { Proj. }\end{array}$ \\
\hline \multicolumn{6}{|l|}{ Balance of payment } \\
\hline Exports of goods and services & 45.2 & 68.3 & 68.3 & 57.2 & 61.4 \\
\hline Imports of goods and services & 60.9 & 77.9 & 78.0 & 63.8 & 63.9 \\
\hline Current account balance, incl. transfers & -10.5 & -8.1 & -11.5 & -12.5 & -14.2 \\
\hline Current account balance, excl. transfers & -21.6 & -14.8 & -16.6 & -16.9 & -18.5 \\
\hline $\begin{array}{l}\text { Gross official reserves (end-of-period, } \\
\text { millions of U.S. dollars) }\end{array}$ & 999 & 1304 & 1299 & 1690 & 1770 \\
\hline $\begin{array}{l}\text { Gross official reserves (weeks of nonaid- } \\
\text { related imports of goods and services) }\end{array}$ & 6.3 & 6.9 & 7.7 & 8.8 & 8.8 \\
\hline \multicolumn{6}{|l|}{ External public debt } \\
\hline Total stock, including IMF & 13705 & 4571 & 4732 & 5595 & 6466 \\
\hline Present value (PV) of debt & 11539 & 3136 & 3526 & 4200 & 4999 \\
\hline $\begin{array}{l}\text { PV of debt (percent of exports of goods and } \\
\text { services) }\end{array}$ & 254.0 & 35.4 & 32.7 & 42.2 & 43.7 \\
\hline \multicolumn{6}{|l|}{ Scheduled debt service } \\
\hline Percent of exports of goods and services & 21.3 & 2.0 & 1.5 & 1.6 & 1.8 \\
\hline Percent of government revenue & 57.3 & 7.1 & 5.4 & 4.0 & 5.2 \\
\hline \multicolumn{6}{|l|}{ Memorandum item: } \\
\hline Nominal GDP (CGF billions) & 9073 & 11875 & 14393 & 16436 & 18970 \\
\hline
\end{tabular}

Sources: Congolese authorities; and IMF staff estimates 


\section{Statement by Kossi Assimaidou, Executive Director for the Democratic Republic of the Congo September 24, 2012}

The authorities of the Democratic Republic of the Congo (DRC) highly appreciate the strong cooperation with the IMF and are thankful to the Executive Board, Management and Staff for the continued assistance provided to their country. In particular, Fund's technical assistance to the DRC has been of critical importance to the improvements in macroeconomic performance. They look forward to continued assistance to help them in their efforts to further strengthen the country's institutional and administrative capacities.

Significant progress in macroeconomic and structural policies has been achieved since the last Article IV consultation. Macroeconomic stability has been enhanced, notably through continued fiscal consolidation, a significant decline in inflation, and continued buildup of international reserves, despite a challenging external environment. In addition, strong efforts have been made in the area of governance and transparency in extractive industries (e.g., over hundred of contracts in the mining sector have been published) albeit few delays related to security concerns. Enhancing the central bank (BCC)'s capacity to meet its core mandate - where advances have also been made - remains in the authorities' structural reform agenda.

The authorities welcome and broadly agree with staff's assessments and analyses. This Article IV consultation - which focuses more on medium-term challenges - comes at an opportune time when the DRC is moving from urgent post-conflict policies to more sustainable economic development. In this vein, the authorities, in consultation with various stakeholders, have prepared a second-generation poverty reduction strategy document (PRSP-2) which, while building on the first PRSP, places more emphasis on growth, employment and the impact of climate change. The strategy seeks to achieve, among others, the Millennium Development Goals (MDGs) by 2020.

\section{RECENT ECONOMIC DEVELOPMENTS AND OUTLOOK}

The assessment that macroeconomic performance in the DRC has been strong during 2010-11 amid a weak global economy and limited external financial support should be recognition of the authorities' sound macroeconomic policies and efforts made in meeting their program objectives. Their actions in ensuring adequate macroeconomic buffers have been underpinned by favorable commodity prices and translated into broadbased activity as the mining sector but also the construction and services sectors have largely contributed to growth since 2010. Inflation continues to be reigned in despite high prices of imported food and fuel. The external sector remains vulnerable due to a number of factors, including high imports of food and capital goods.

On the fiscal front, significant progress has been made. Fiscal consolidation has been the main objective, with strong measures taken to restrain spending. At the same time, structural reforms to enhance public financial management and natural resource management have been undertaken and are being pursued. The steps taken, including the 
commitment to zero (net) financing from the BCC has brought the fiscal deficit down from $2 \frac{1}{2}$ percent of GDP in 2009 to a projected deficit of 0.9 percent of GDP in 2012, and has been instrumental in lowering inflation and stopped the inflation-exchange rate depreciation cycle. For 2012, the budget has been elaborated by the authorities to respond to the tremendous needs facing the Congolese economy and population. Nevertheless, for the rest of 2012, the authorities will execute spending only as revenue becomes available in accordance with clearly set priorities.

Technical support from the IMF, the World Bank and other partners has been instrumental in the progress made on some structural fronts, notably public finance reforms (through the Strategic Plan for Public Finance Reform), fiscal planning, macrofiscal forecasting, steps toward a medium-term budget framework, and governance measures in the natural resource sector. Efforts by the BCC are also underway to strengthen financial supervision, deepen banking sector development, enhance its own financial situation through a recently completed pension reform, and eliminate non-core activities such as the management of a hospital center and real estate.

Despite a relatively positive short-term outlook, the DRC authorities recognize that the tail risks facing this economy-weaker global demand, falling commodity prices, and even an aggravated conflict in eastern provinces - argue for further strengthening macroeconomic buffers. They have drawn lessons from the experience of the global financial crisis that hit the country's economy in 2009, and continue to build a stronger position to absorb external shocks. On the external front, the DRC authorities will strive to eliminate inefficient public investment financed by external resources and which accentuate the high debt risk.

\section{MEDIUM-TERM POLICIES}

\section{Poverty Reduction Strategy for 2011-15}

The authorities' actions over the medium term will be guided by their new PRSP. Building on macroeconomic stability and good economic performance of recent years, the authorities will implement a poverty reduction strategy based on an ambitious medium-term framework that would be underpinned by greater expansion of the natural resource and agriculture sectors as well as efforts to enhance infrastructure, notably in the transport, energy and telecommunication sectors.

Employment is at the core of the country's strategy. In implementing this strategy, particular emphasis is being put on demographic, education, health and social protection issues, with the view to strengthen human capital. At the same time, actions to further improve governance and the business environment are needed to attract private sector investment.

My authorities are appreciative of the encouragements from Fund and World Bank staffs, and welcome their recommendations to strengthen the PRSP-2, including in the areas of macroeconomic policies, institutional capacities, sectoral priorities, resource allocations, and implementation risk. 


\section{Establishing a Fiscal Policy Anchor}

The authorities view the zero central bank financing of the budget as a key objective in managing public finances. They will continue to control expenditures and make full use of the recent fiscal reforms (value-added tax, strengthened capacity of the large taxpayer unit, one-stop customs window, improved information technology systems) to raise highly needed domestic revenue.

The DRC authorities look forward to continued Fund technical assistance to help them realize the potential of the natural resource sector, further improve public financial management and enhance the quality of spending. Regarding the fuel price mechanism, they intend to reform it, fully cognizant of its propensity to be subjected to political pressures and to generate volatile fuel prices and tax revenue. They are reflecting on the automatic price-smoothing mechanisms proposed by staff, which have significant advantages over the current policy in terms of retail price volatility and fiscal costs.

\section{Enhancing the Effectiveness of Monetary Policy}

The authorities welcome staff's assessment of the factors that hamper the efficacy of monetary policy, and share such evaluation. They confirm their commitment to the current monetary and exchange rate regime which is better adapted to the country's circumstances and necessary ability to absorb exogenous shocks. They share the recommendations detailed in Annex VIII of the report, aimed at strengthening the independence of the central bank, enhancing its data and analytical capacity, deepening financial markets and encouraging de-dollarization.

\section{Structural Reforms to Bolster Economic Resilience}

The DRC economy remains vulnerable to external shocks but also to internal developments. The authorities agree on the need to enhance both the resilience of the financial sector and the business environment. They welcome staff's recommendations in this regard and they intend to implement them with technical assistance from the Fund and other partners. In particular, regarding the financial sector, the authorities requested a financial sector evaluation under FSAP and will pursue stress testing to assess vulnerabilities. They will also submit to parliament a new payment systems law aimed at reducing transaction costs and developing the banking sector.

On extractive industries, the authorities have already implemented a number of measures supported by the World Bank to strengthen governance in the natural resource sector, and they have submitted the instruments to adhere to OHADA (Organization for the Harmonization of African Business Law). The authorities will continue to build on the good progress made in ensuring the sanctity of contracts and private property rights and reviewing the mining code, to complete the governance and transparency agenda. They remain committed to the timely publication of natural resource contracts. They have expressed their intention to put in place measures to make commercially-run SOEs accountable to the government as their sole shareholder, strengthen the legal and regulatory framework applicable to the governance of those enterprises, and limit their 
external financing to concessional resources with the view to safeguarding public debt burden.

In conclusion, I would like to reaffirm my DRC authorities' determination to pursue steadfastly policies that are conducive to macroeconomic stabily, poverty reduction and inclusive growth. They will continue to work closely with their development partners and the Fund in the achievement of the objectives spelled out in their PRSP. 